\title{
CONFIDENCE INTERVALS FOR SOME FUNCTIONS \\ OF SEVERAL BERNOULLI PARAMETERS WITH RELIABILITY APPLICATIONS ${ }^{1}$ \\ by \\ Dar-Shong Hwang and Robert J. Buehler \\ Technical Report No. 161
}

November 1971

University of Minnesota
Minneapolis, Minnesota

$1_{\text {This }}$ research was supported by National science Foundation Grant GP-24183. 


\title{
CONFIDENCE INTERVALS FOR SOME FUNCTIONS \\ OF SEVERAL BERNOULLI PARAMETERS \\ WITH RELIABILITY APPLICATIONS ${ }^{1}$
}

By

\author{
Dar-Shong Hwang and Robert J. Buehler \\ University of Minnesota
}

$1_{\text {This }}$ research was supported by National Science Foundation Grant GP-24183. 
ABSTRACT

In reliability theory one is often interested in the estimation of products and other functions of Bernoulli parameters. Previous work has been mainly concerned with binomial data. In the present paper other sampling methods are considered, negative binomial in particular. It is shown that the theory of exponential families can be used to obtain exact confidence limits for products and quotients of Bernoulli parameters when negative binomial observations are available from each population. For the case of two populations the relevant distributions are related to hypergeometric functions. To estimate more general functions such as sums of products of Bernoulli parameters, some sampling methods are suggested which are based on theory of compound distributions. Another method of obtaining exact confidence limits for products is given which exploits the independence of the minimum and difference of independent geometric variates. This last method is a discrete analog of a procedure due to Lieberman and Ross for estimating sums of scale parameters of exponential distributions. 


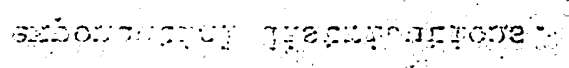

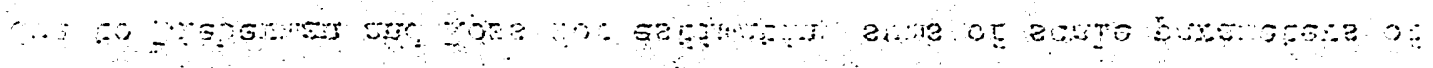

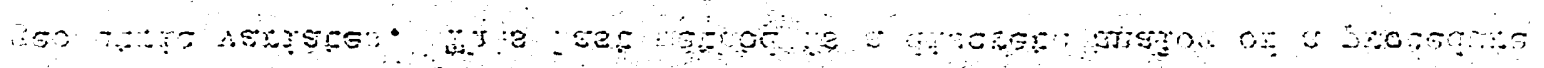

7. \%

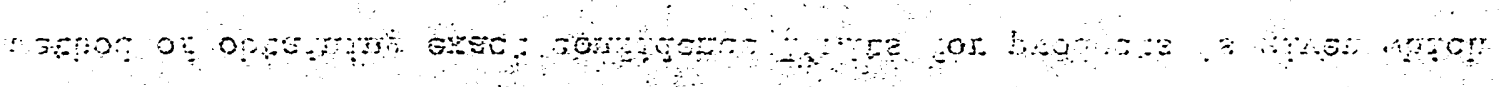

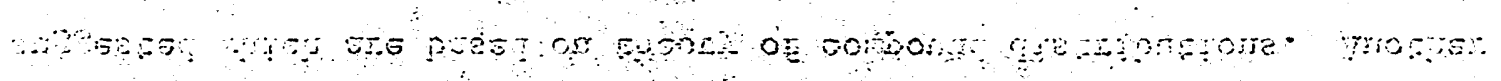

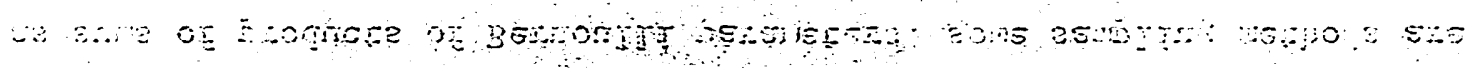

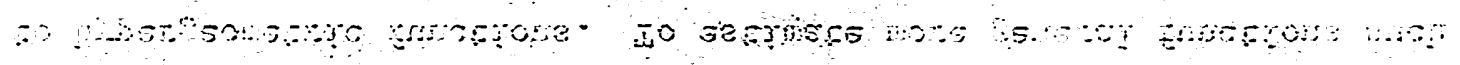

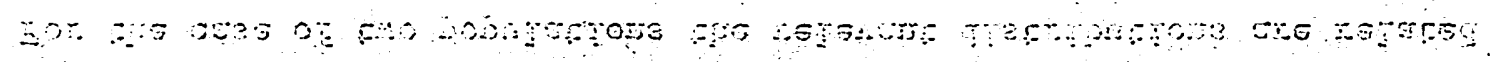

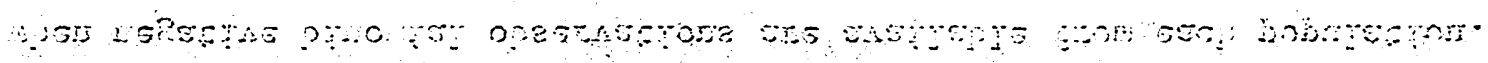

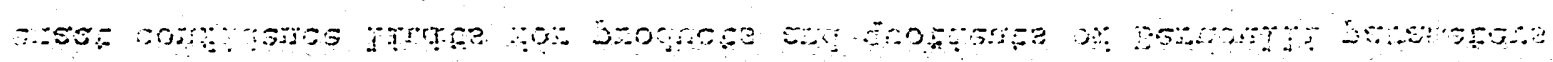

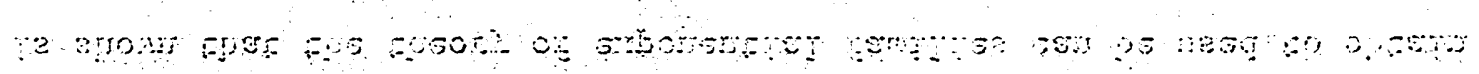

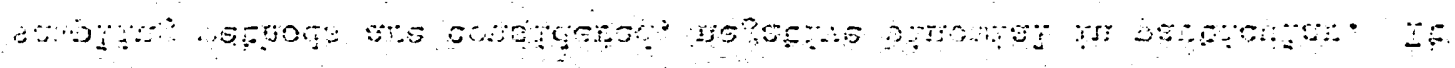

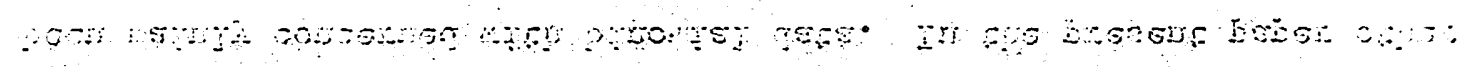

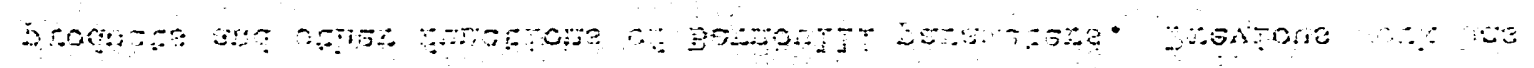

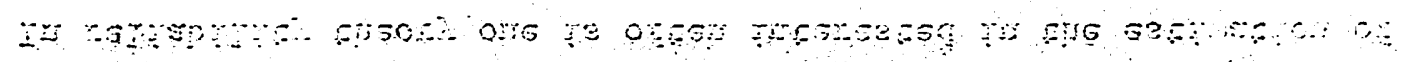

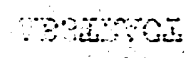

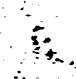




\section{Introduction.}

Let $\pi_{1}, \pi_{2}, \ldots$, denote Bernoulli populations with parameters $p_{1}, p_{2}, \ldots$ We are concerned here with interval estimation of various functions of the parameters, in particular, products, quotients, and sums of products. Most previous work in this area has been concerned with data in the form of binomial observations. The present paper shows that other sampling methods (negative binomial, for example) lead to new and possibly advantageous ways of obtaining confidence intervals. In some cases, sampling rules are suggested which depend upon the particular parametric function to be estimated.

\subsection{Practical applications.}

Our motivation comes partly from reliability theory and partly from biomedical applications, In reliability theory $\mathrm{P}_{\mathbf{j}}$ may denote the reliability of a component from population $\pi_{j}$. Then a system made up of one component from each of $k$ populations has reliability

(1.1) $\quad \mathrm{R}_{1}=\mathrm{p}_{1} \mathrm{p}_{2} \cdots \mathrm{p}_{\mathrm{k}}$

if the components are in series, or reliability

(1.2) $\quad R_{2}=1-q_{1} q_{2} \cdots q_{k} \quad\left(q_{i}=1-p_{i}\right)$

if they are in parallel. Other systems whose reliabilities are more general polynomials in the $p_{i}$ are also of interest. In any case we wish to estimate the system reliability using data pertaining to the individual components.

Interval estimation of $R_{1}$ or $R_{2}$ (or special cases) has been considered by various writers. Buehler [2] and Harris [5] use Poisson approximations to estimate $R_{2}$. Madansky [12] and Myhre and Saunders 


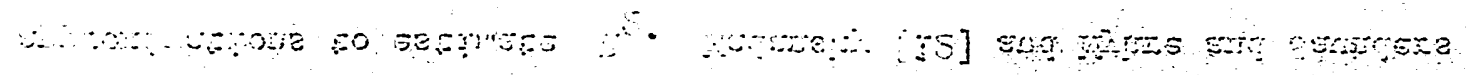

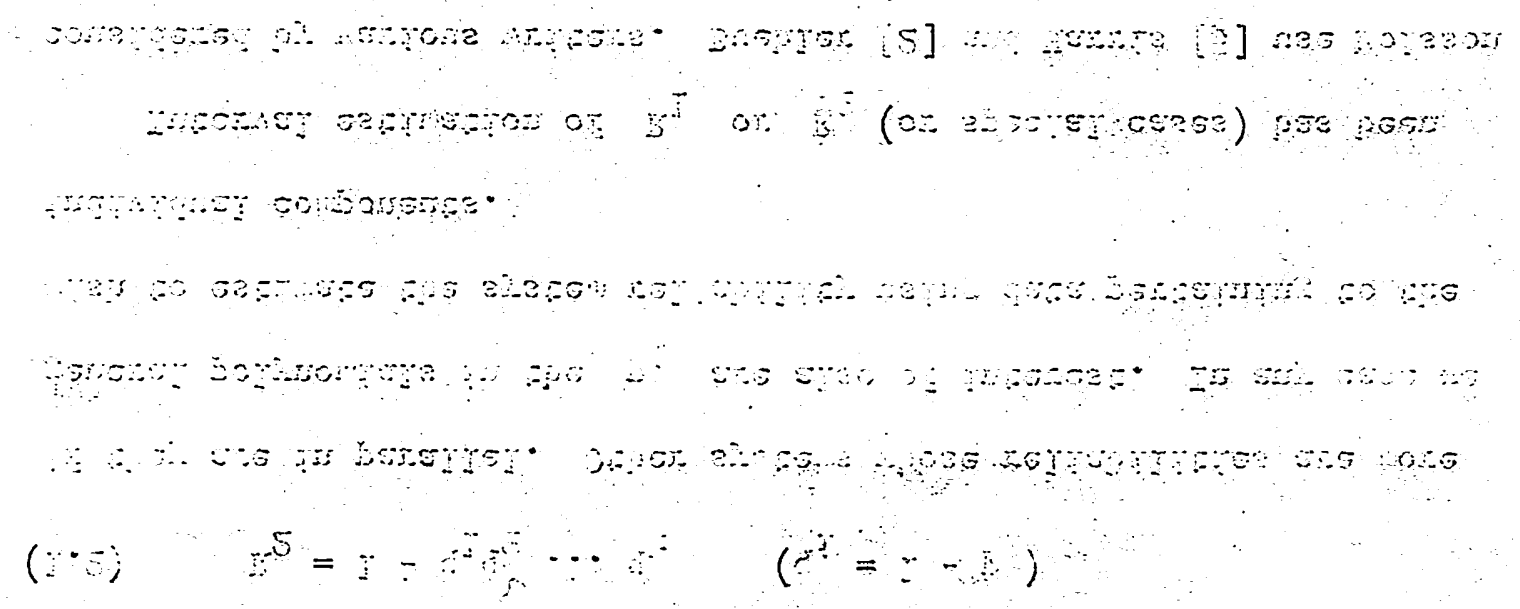


[14] estimate $R_{1}$ and $R_{2}$ by a chi-square approximation. Other work is reviewed by Rosenblatt [18] and by Mann [13].

Biomedical applications of the methods of section 2 below have been considered by Hwang [6]. These include: (i) the estimation of the difference of two bacterial densities by the dilution method; (ii) comparison of two Yule's birth processes; and (iii) estimation of ratios and cross ratios of proportions, which arise, for example, in the theory of effectiveness indices. We hope to consider these in a later paper.

\subsection{Nature of the difficulties.}

In general we wish to find confidence limits for a given function of $k$ Bernoulli parameters. Of course, large sample methods will always yield approximate solutions, but the present paper is concerned with mathematical devices for obtaining "exact" solutions despite the presence of $k-1$ nuisance parameters.

The second difficulty arises from the discreteness of the distributions. For any confidence level $\gamma$, it is possible to give intervals with probability of coverage exactly equal to $\gamma$ only by artificial randomization (see for example [19] or Section 3.5 of [8].). We prefer the usual "conservative" solutions having coverage probability greater than or equal to $\gamma$. But such solutions may justifiably be criticized if they are in a sense, too conservative. One quantitative measure of this would be the probability of coverage averaged over the parameter space. Hopefully this average should be only slightly greater than $\gamma$; but for discrete distributions having all (or nearly all) of the probability concentrated on only a few mass points, it may be closer to unity. It 


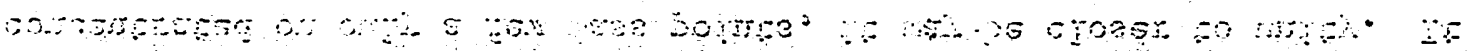

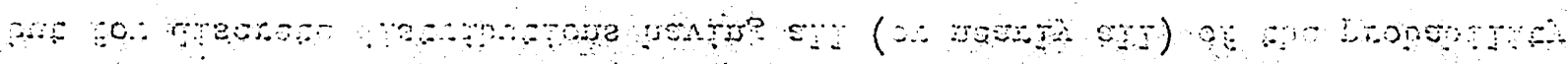

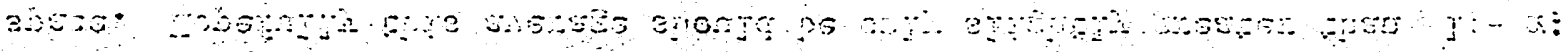

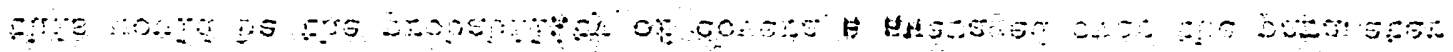

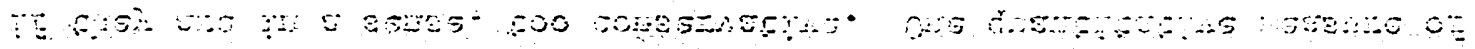

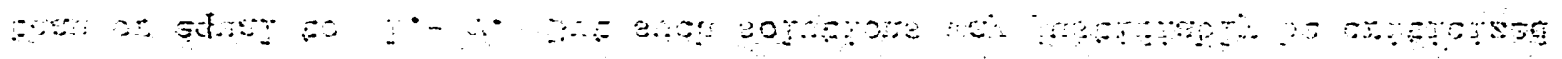

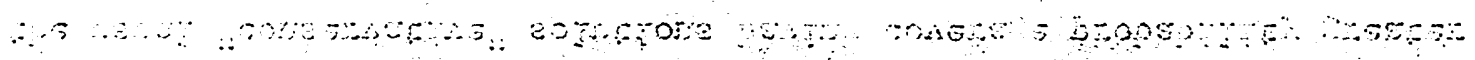

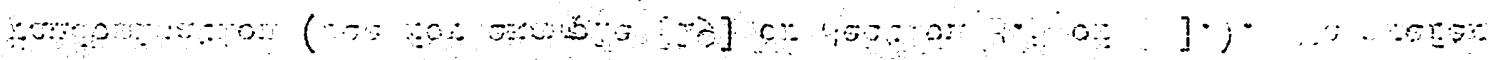
कo

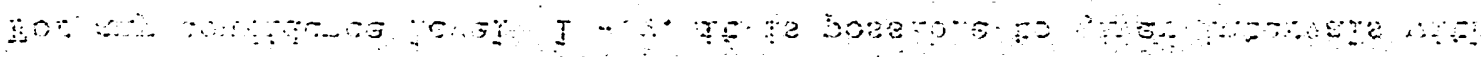
$\therefore$ e 2500

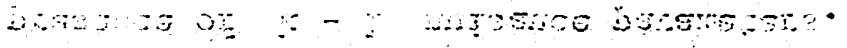

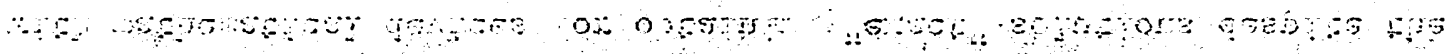
on

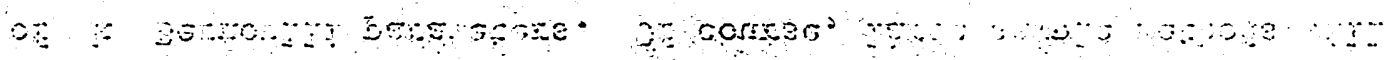

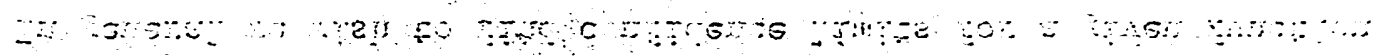
Z 000500

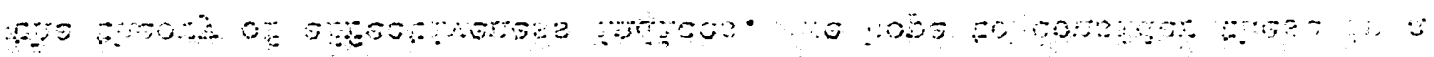

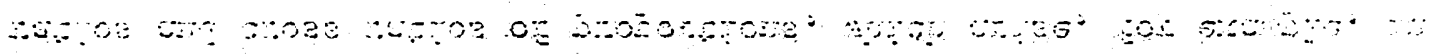

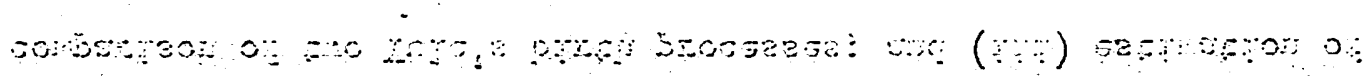

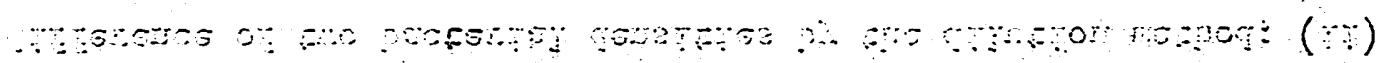

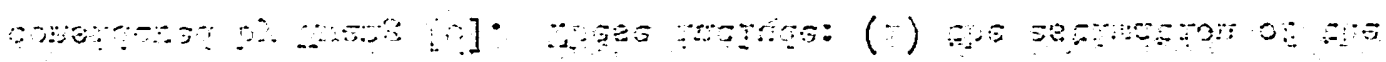
भor

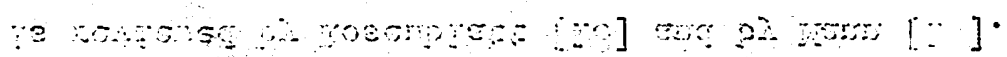

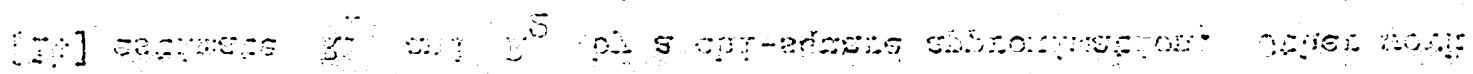


will be convenient to have an expression for this undesirable state of affairs, and we will speak in a qualitative way of situations having a high "discreteness index" to indicate solutions which are highly "conservative." Examples would be a binomial distribution with small $n(n=3$, say), or a Poisson distribution where prior information would indicate a mean small compared to unity.

A third difficulty is that in reliability applications in particular, the parameters $p_{j}$ may be close to unity. This fact may have undesirable consequences for the discreteness index or for the expected sample size with certain sampling schemes.

It is probably impossible to deal simultaneously with all these difficulties in a completely satisfactory way, at least within the NeymanPearson framework. Nevertheless we hope that the methods given below will find a number of legitimate applications.

\subsection{Summary.}

In Section 2 we show that inverse (i.e., negative) binomial sampling allows us to use the Lehmann-Scheffé theory of exponential families to estimate products and quotients of Bernoulli parameters. Formulas are given for the most general case and for a number of special cases. Section 3 describes a new sampling method which is specifically aimed at the estimation of certain parametric functions not covered in section 2 . Section 4 gives a number of ways of using properties of compound Poisson distributions to estimate sums of products of Bernoulli parameters. In Section 5 we show that a method due to Lieberman and Ross for estimating sums of scale parameters of exponential distributions can be modified so that it applies also to problems involving products of Bernoulli parameters. 
30 a +

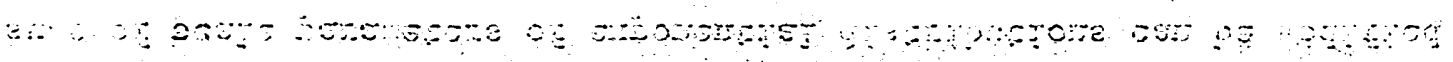

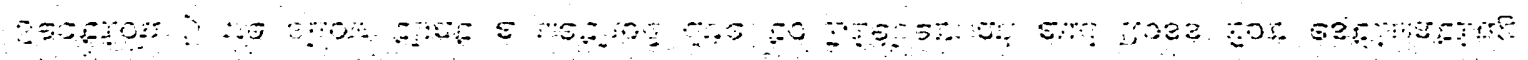

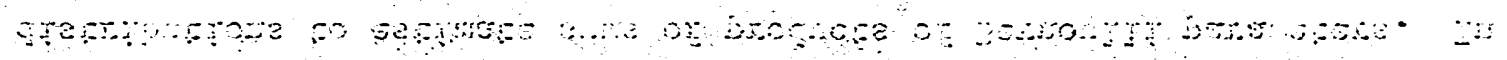
a

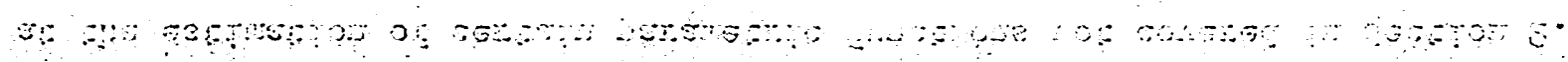

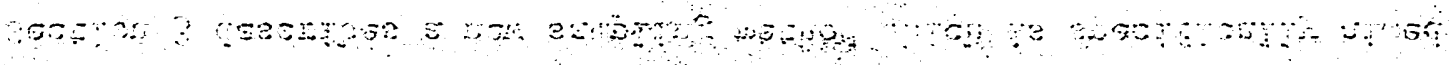

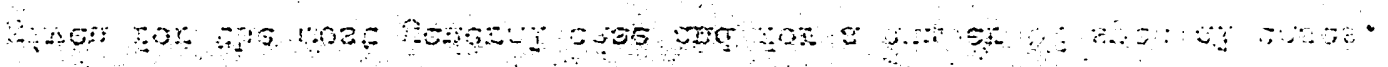

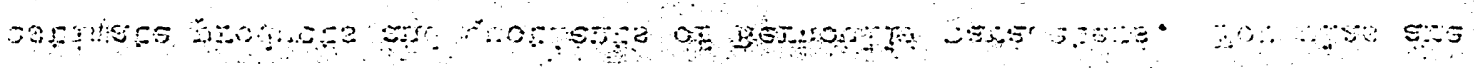

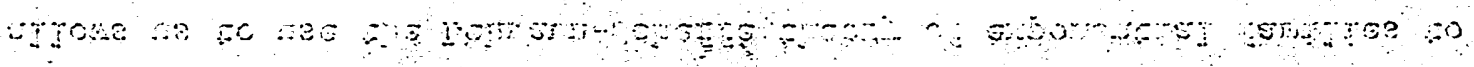

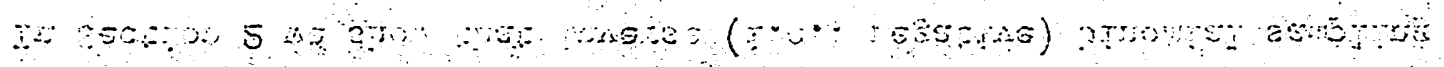
$\because \quad \because n$

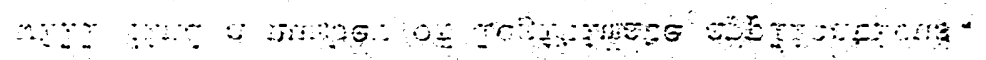

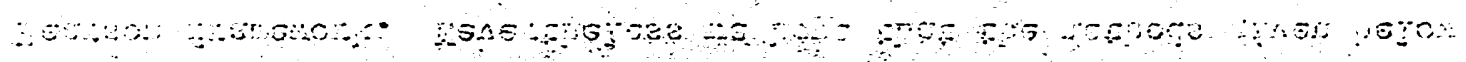

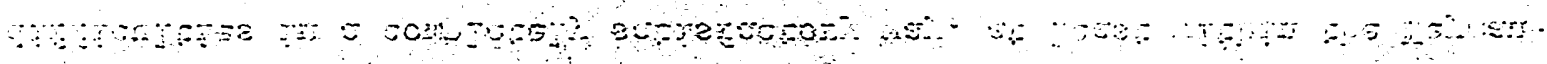

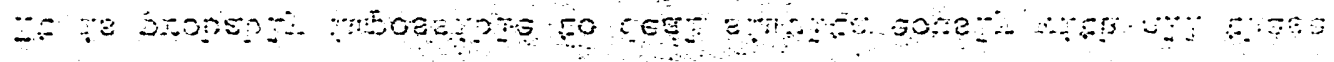

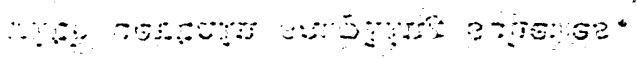

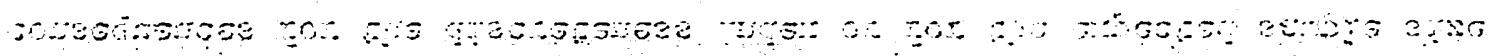

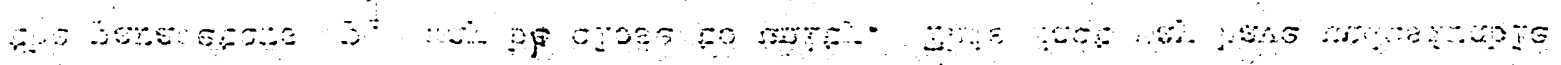
\% 00000000000

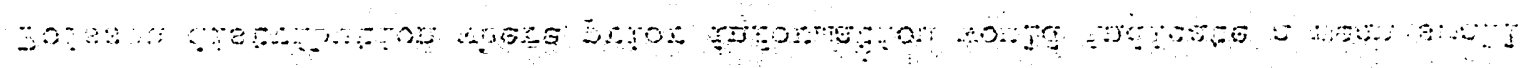
жан

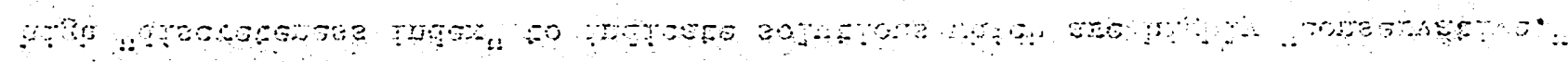
-

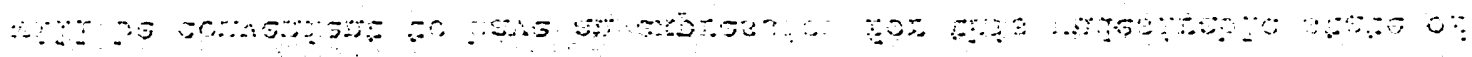


2. Distribution Theory for Estimation of Products and Quotients Using

Inverse Binomial Sampling.

In this section we exploit the theory of exponential families to remove nuisance parameters when the probability models are negative binomial. Other reliability applications of the same theory have been made by Lentner and Buehler [10] for gamma models and by Harris [5] for Poisson models.

\subsection{The general case.}

Let a Bernoulli population have probability $p$ of success and for any fixed $r=1,2, \ldots$, let $x$ denote the number of successes observed before the $r^{\text {th }}$ failure. Then $X$ has the negative binomial distribution (2.1) $\quad P\{X=x\}=\left(\begin{array}{c}r+x-1 \\ x\end{array}\right) p^{x}(1-p)^{r} \quad x=0,1,2, \ldots$ which we will abbreviate by (2.2) $\quad X \sim N B(r, p)$.

If we sample independently from $k+k^{\prime}$ Bernoulli populations to obtain random variables $x_{i} \sim N B\left(r_{i}, p_{i}\right)$ for $i=1, \ldots, k$ and $Y_{j} \sim N B\left(s_{j}, P_{j}^{\prime}\right)$ for $j=1, \ldots, k^{\prime}$, then it will be seen to be possible to obtain confidence intervals for the function

$$
\text { (2.3) } \quad \theta=p_{1} p_{2} \cdots p_{k} / p_{1}^{\prime} p_{2}^{\prime} \cdots p_{k^{\prime}}^{\prime}
$$

The essential reasons are that the distribution (2.1) has the exponential form

$$
(2.4) \quad g(\varphi) h(x) e^{\varphi x}
$$

where 


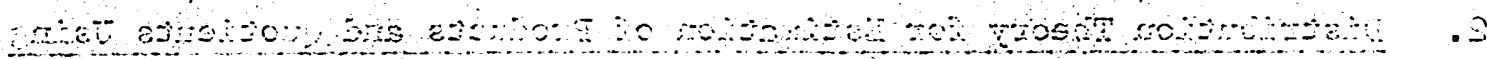

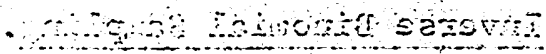

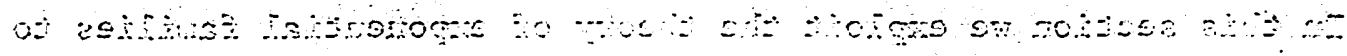

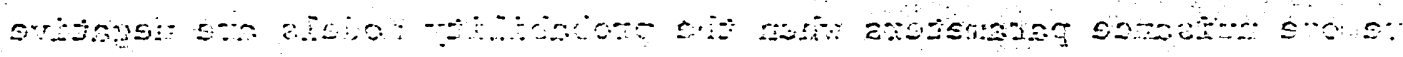

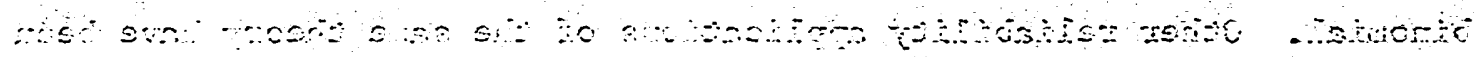

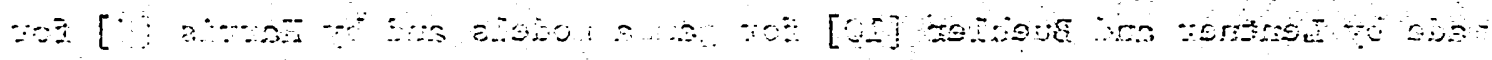

$$
\begin{aligned}
& \text { - astori mosion }
\end{aligned}
$$

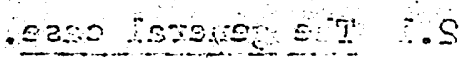

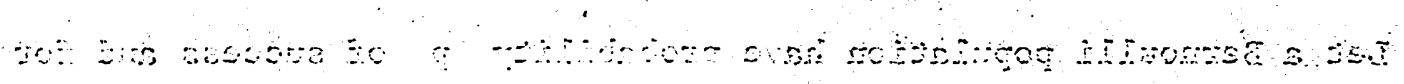

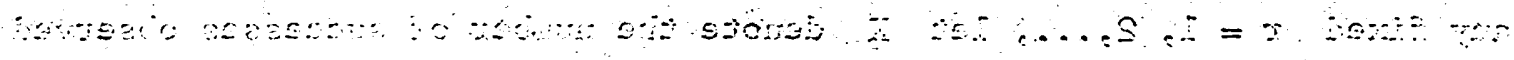

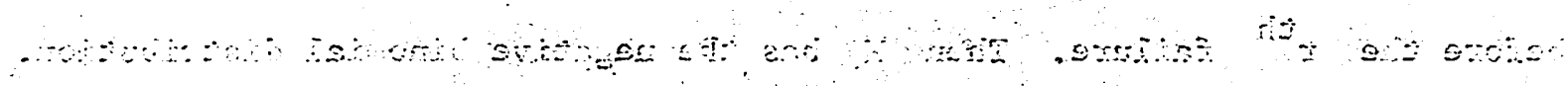

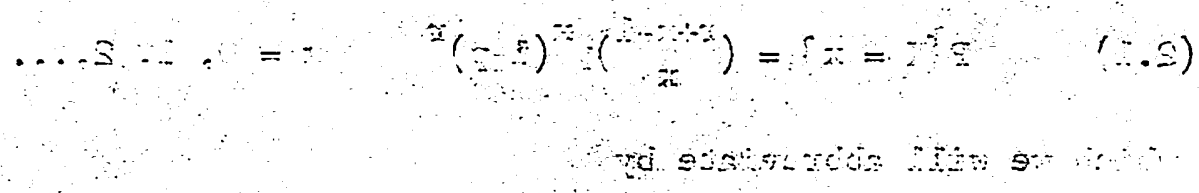

$$
\because(\because, \because)-\because
$$

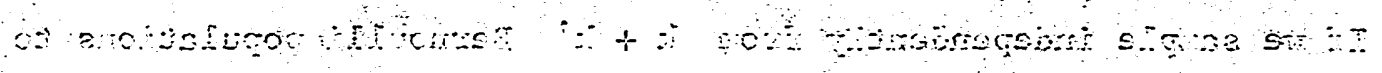

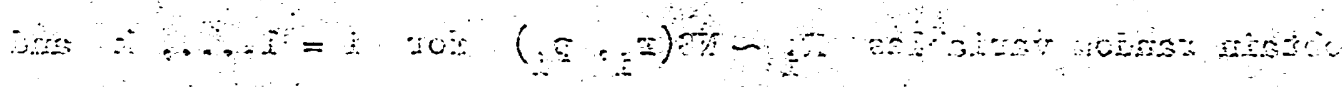

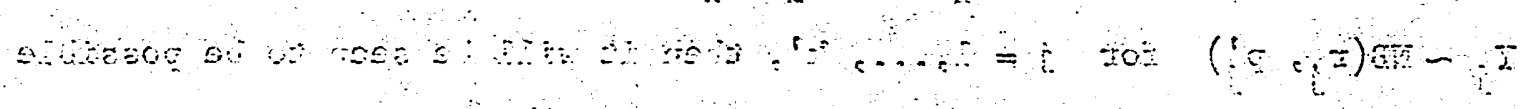

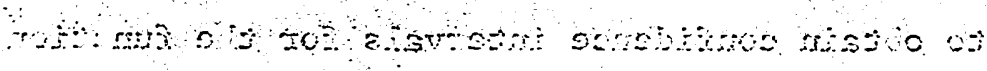

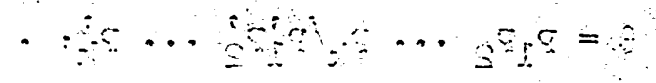

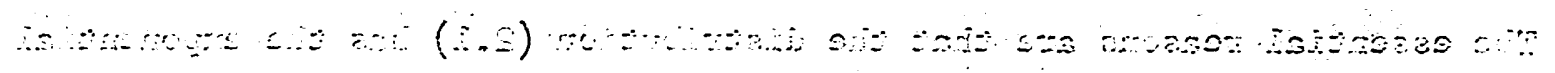
$\$ 2$

$$
\Leftrightarrow(-2) \quad(6)
$$




$$
\varphi=\log p, g(\varphi)=\left(1-e^{\varphi}\right)^{r}, h(x)=\left(\begin{array}{c}
r+x-1 \\
x
\end{array}\right)
$$

and that $\log \theta$ is a linear function of $\varphi_{i}=\log p_{i}$ and $\varphi_{j}^{\prime}=\log p_{j}^{\prime}$. To apply the theory of Lehmann and Scheffé [9] (or see Sec. 4.4 of [8]), we transform the random variables by

$$
\begin{array}{ll}
w_{1}=x_{1} & \\
w_{i}=x_{i}-x_{1} & \text { for } \quad i=2,3, \ldots, k, \\
v_{j}=Y_{j}+x_{1} & \text { for } \quad j=1,2, \ldots, k^{\prime} .
\end{array}
$$

Let $U=\left(w_{2}, w_{3}, \ldots, w_{k}, v_{1}, v_{2}, \ldots, v_{k^{\prime}}\right)$ and $u=\left(w_{2}, w_{3}, \ldots, w_{k}\right.$, $\left.v_{1}, v_{2}, \ldots, v_{k^{\prime}}\right)$. Then it is straightforward to verify that

(2.7) $\quad P\left\{\mathrm{~W}_{1}=\mathrm{w}_{1}, \mathrm{U}=\mathrm{u}\right\}=\mathrm{A}(\mathrm{u}) \mathrm{B}\left(\mathrm{w}_{1}, \mathrm{u}\right) \theta^{\mathrm{W}}{ }^{\mathrm{w}}$

where $\theta$ is defined by (2.3),

$$
A(u)=q_{1}^{r_{1}} \underset{i=2}{k} \prod_{j=1}^{k^{\prime}} q_{i}^{r_{i}} p_{i}^{w_{i}} q_{j}^{\prime}{ }_{j} p_{j}^{\prime} v_{j}
$$

$$
B\left(w_{1}, u\right)=\left(\begin{array}{c}
r_{1}+w_{1}-1 \\
w_{1}
\end{array}\right) \prod_{i=2}^{k} \prod_{j=1}^{k^{\prime}}\left(\begin{array}{c}
r_{i}+w_{i}+w_{1}-1 \\
w_{i}+w_{1}
\end{array}\right)\left(\begin{array}{c}
s_{j}+v_{j}-w_{1}-1 \\
v_{j}-w_{1}
\end{array}\right)
$$

$$
q_{i}=1-p_{i}, q_{j}^{\prime}=1-p_{j}^{\prime},
$$

and the range of the variables is

$$
\begin{aligned}
& w_{1}=0,1,2, \ldots \\
& w_{i}=-w_{1},-w_{1}+1,-w_{1}+2, \ldots \\
& v_{j}=w_{1}, w_{1}+1, w_{1}+2, \ldots .
\end{aligned}
$$

From (2.7) we obtain the conditional distribution

$$
P\left\{W_{1}=w_{1} \mid U=u\right\}=B\left(w_{1}, u\right) \theta^{W_{1}} / \sum_{t} B(t, u) \theta^{t}
$$

where the possible values of $w_{1}$ are $\max \left\{0, \max \left(-w_{2}, \ldots,-w_{k}\right)\right\} \leq w_{1}$ $\leq \min \left(v_{1}, \ldots, v_{k^{\prime}}\right)$, and the values of $t$ in the summation are the same 


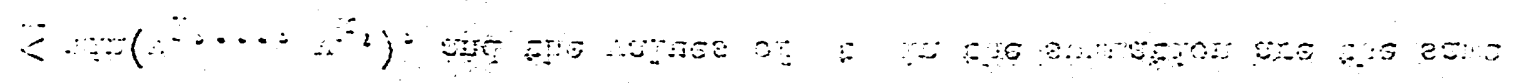

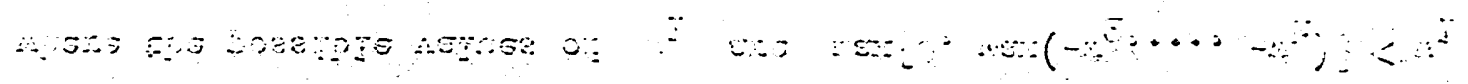

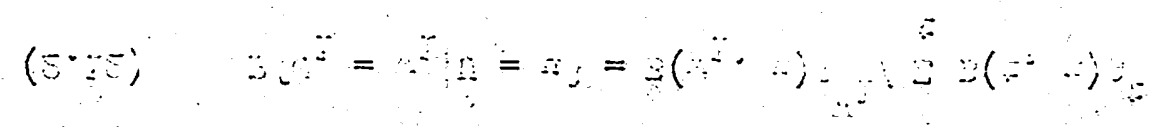

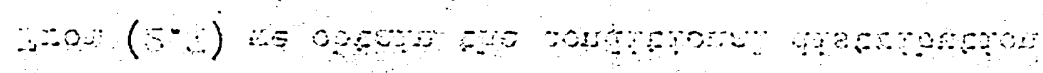

$$
\begin{aligned}
& \therefore=r+n^{4}+\cdots \cdots
\end{aligned}
$$

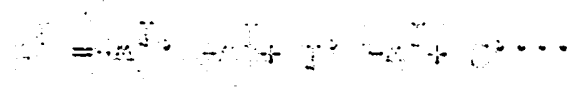

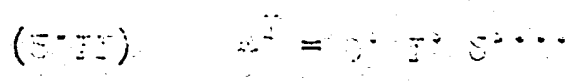

$$
\begin{aligned}
& \text { ar, o }
\end{aligned}
$$

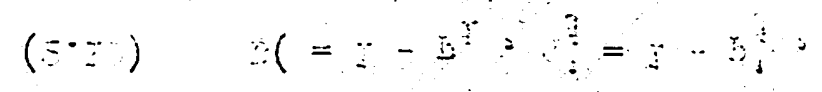

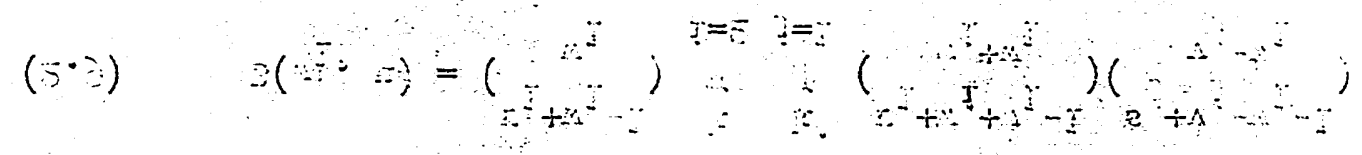

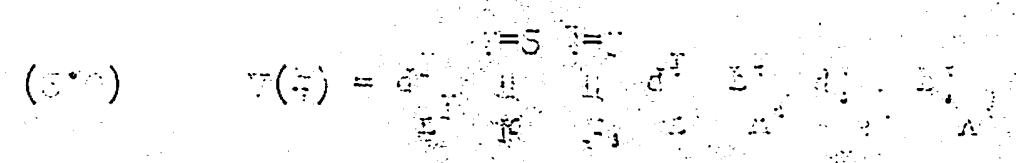

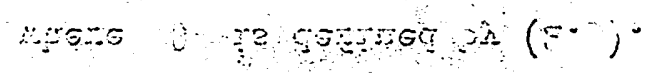

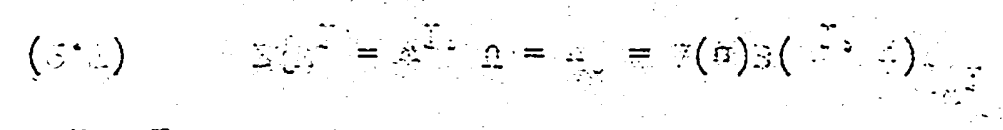

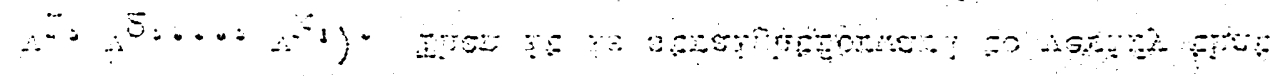

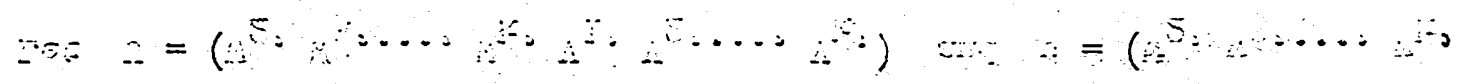

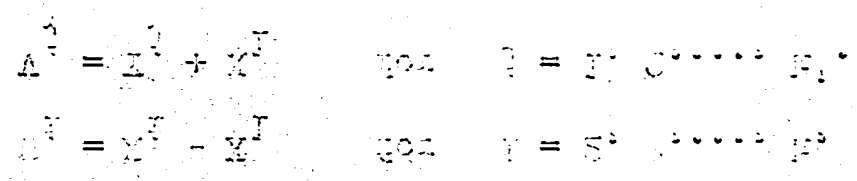$$
(3) \quad \ddot{z}=\ddot{q}
$$

$\therefore$ a : a

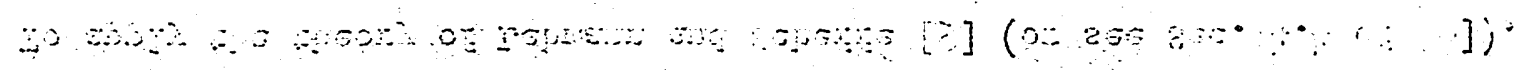

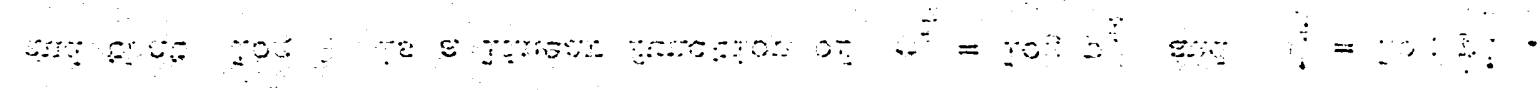

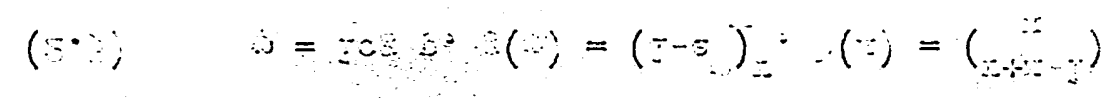


as the possible values of $w_{1}$. Thus by using known theory of exponential families we have obtained a conditional distribution (2.12) which depends on the $k+k^{\prime}$ parameters only through the function $\theta$. Confidence intervals can be obtained from (2.12) in the usual way. For any confidence level $\gamma$, and any observation $w_{1}=w_{1}, U=u$, an upper confidence limit $\theta_{2}\left(w_{1}, u\right)$ is defined by

$$
\theta_{2}\left(w_{1}, u\right)=\sup \left\{\theta: \sum_{t \leq w_{1}} P\left\{w_{1}=t \mid u=u\right\} \geq 1-\gamma\right\} .
$$

Similarly a lower confidence limit $\theta_{1}\left(w_{1}, u\right)$ is defined by

$$
\theta_{1}\left(w_{1}, u\right)=\inf \left\{\theta: \sum_{t \geq w_{1}} P\left\{w_{1}=t \mid u=u\right\} \geq 1-\gamma\right\} \text {. }
$$

Because of the discreteness of the distribution these are "conservative" intervals satisfying the inequalities

$$
\mathrm{P}\left\{\theta \leq \theta_{2}\left(\mathrm{w}_{1}, \mathrm{u}\right) \mid \mathrm{U}=\mathrm{u}\right\} \geq \gamma \text { for all } \theta, \mathrm{u}
$$

$$
\mathrm{P}\left\{\theta \geq \theta_{1}\left(\mathrm{w}_{1}, \mathrm{u}\right) \mid \mathrm{U}=\mathrm{u}\right\} \geq \gamma \text { for a11 } \theta, \mathrm{u} \text {. }
$$

If randomization is used to make the probabilities equal to $\gamma$, the resulting solutions are known to be "uniformly most accurate unbiased" (see Chapter 4 of $[8])$.

\subsection{The product of $\mathbf{k}$ parameters.}

In this section we give results which are appropriate for the special case when $\theta$ in (2.3) is replaced by

$$
\theta^{*}=\mathrm{p}_{1} \mathrm{p}_{2} \ldots \mathrm{p}_{\mathrm{k}}
$$

We then simply drop the variates $Y_{j}$ and $V_{j}$ and redefine $U$ and $u$ by $U=\left(w_{2}, w_{3}, \ldots, w_{k}\right), u=\left(w_{2}, w_{3}, \ldots, w_{k}\right)$. The conditional distribution (2.12) then becomes 


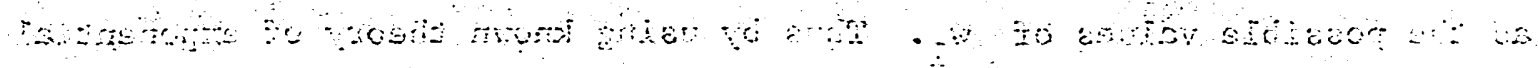
(2)

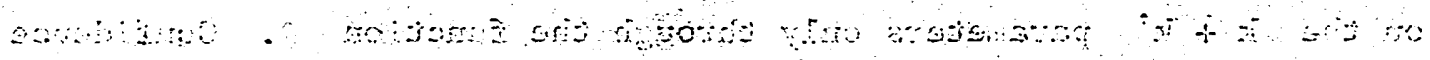

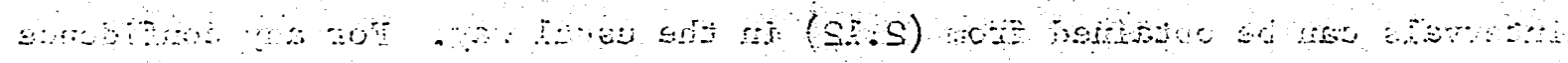

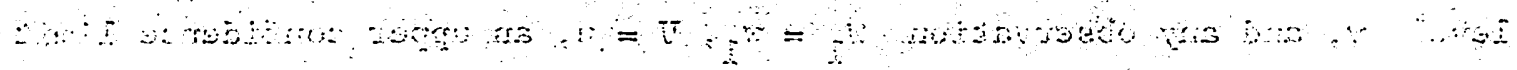

$$
\begin{aligned}
& \text { तestas } \quad(\because-y)
\end{aligned}
$$

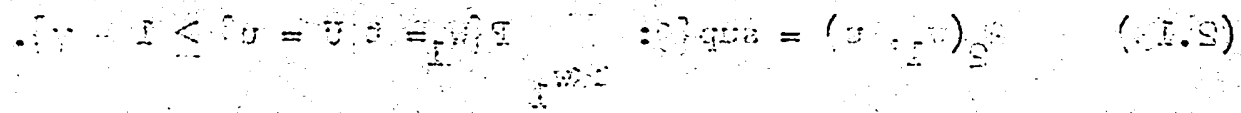

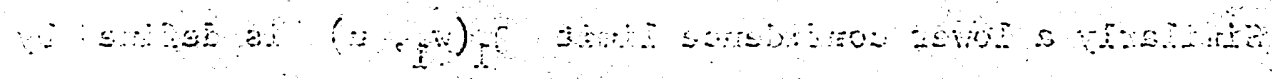

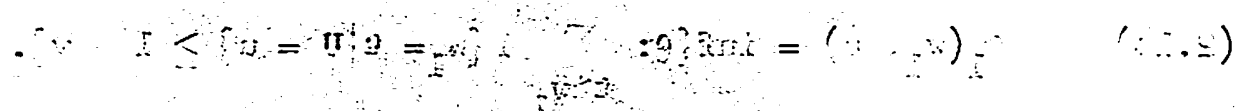

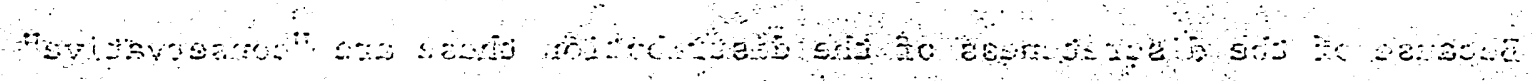

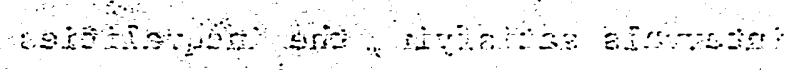

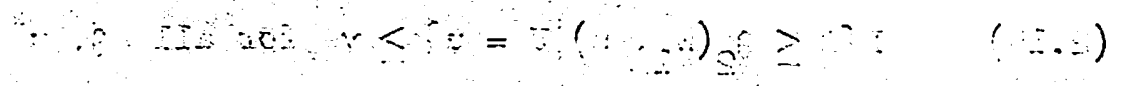

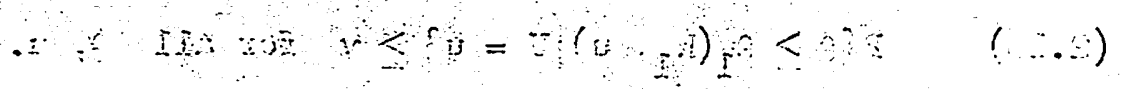

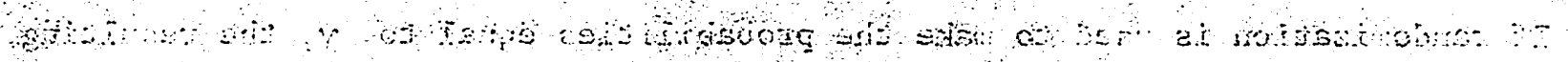

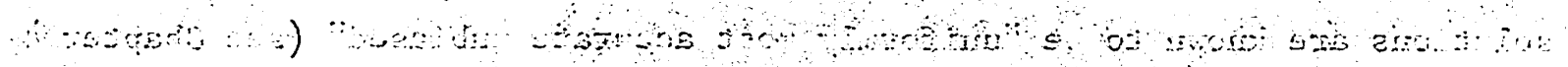

$$
\begin{aligned}
& \cdot(5)
\end{aligned}
$$

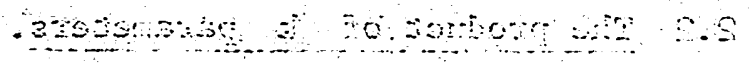

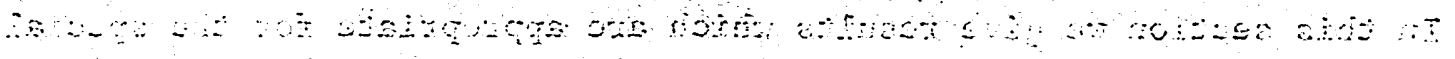
(2)

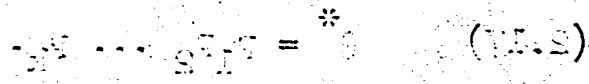

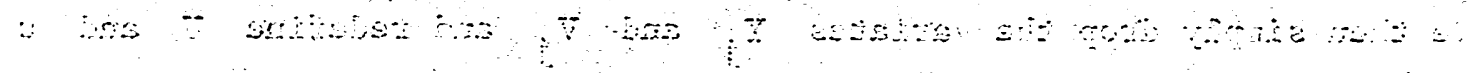

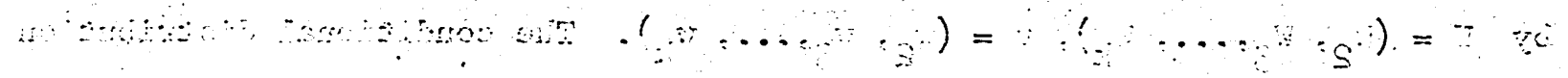

$$
\begin{aligned}
& \text { asos (as) }
\end{aligned}
$$




$$
P\left\{W_{1}=w_{1} \mid U=u\right\}=B^{*}\left(w_{1}, u\right)\left(\theta^{*}\right)^{W_{1}} / \sum_{t} B^{*}(t, u)\left(\theta^{*}\right)^{t}
$$

where

$$
B^{*}\left(w_{1}, u\right)=\left(\begin{array}{c}
r_{1}+w_{1}-1 \\
w_{1}
\end{array}\right) \prod_{i=2}^{k}\left(\begin{array}{c}
r_{i}+w_{i}+w_{1}-1 \\
w_{i}+w_{1}
\end{array}\right)
$$

and the range of $w_{1}$ (and $t$ ) is $\max \left\{0,-w_{2}, \ldots,-w_{k}\right\} \leq w_{1}<\infty$. We now show some relationships between these expressions and hypergeometric functions .

\subsection{The product of two parameters.}

The usual definition of the hypergeometric function is [20]:

$$
\begin{aligned}
F(a, b, c ; z)= & \left\{1+\frac{a \cdot b}{1 \cdot c} z+\frac{a(a+1) b(b+1)}{1 \cdot 2 \cdot c(c+1)} z^{2}\right. \\
& \left.+\frac{a(a+1)(a+2) b(b+1)(b+2)}{1 \cdot 2 \cdot 3 \cdot c(c+1)(c+2)} z^{3}+\ldots\right\} .
\end{aligned}
$$

Let us define

$$
f(a, b, c ; z, n)=\frac{\Gamma(c)}{\Gamma(a) \Gamma(b)} \frac{\Gamma(a+n) \Gamma(b+n)}{\Gamma(c+n)} \frac{z^{n}}{n !},
$$

where $\Gamma(x)$ is the gamma function. Then it is straightforward to verify that

$$
\text { (2.22) } \quad F(a, b, c ; z)=\sum_{n=0}^{\infty} f(a, b, c ; z, n) \text {. }
$$

If we further define

$$
\begin{aligned}
& \text { (2.23) } F_{x}(a, b, c ; z)=\sum_{n=0}^{X} f(a, b, c ; z, n), \\
& \text { then } F_{\infty}(a, b, c ; z)=F(a, b, c ; z) .
\end{aligned}
$$$$
\text { Going back to (2.18), and taking the special case } k=2 \text {, replacing }
$$

$$
\theta^{*} \text { by }
$$

(2.24) $\quad \lambda=\mathrm{P}_{1} \mathrm{p}_{2}$ 


$$
\begin{aligned}
& y=0.0 \\
& \because 2 \\
& \text { opm por }
\end{aligned}
$$

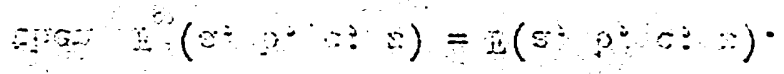

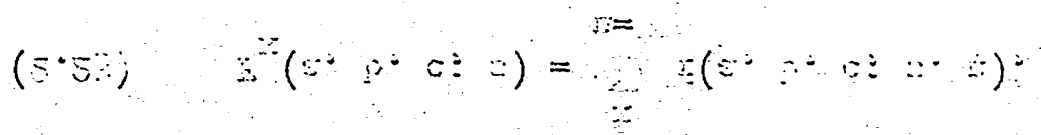

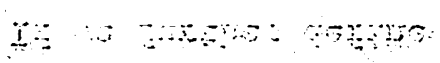

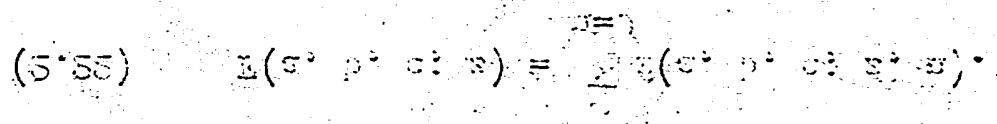

$$
\begin{aligned}
& 50
\end{aligned}
$$

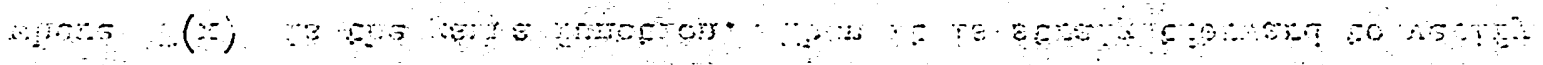

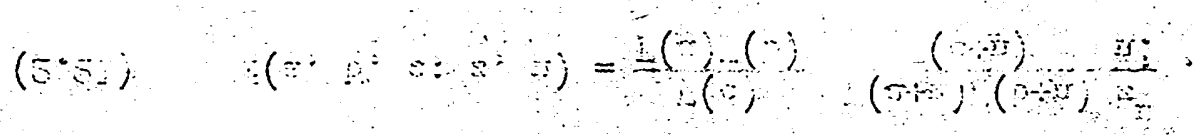

$$
\begin{aligned}
& 258 \quad 20 \%
\end{aligned}
$$

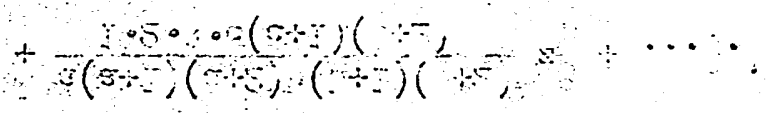

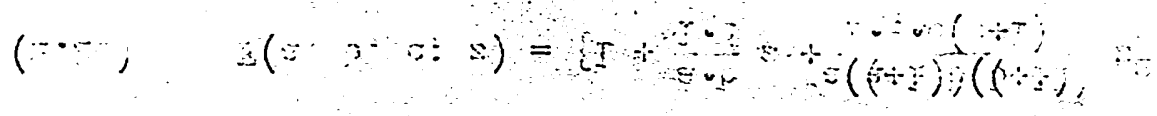

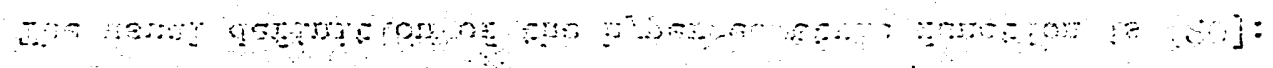

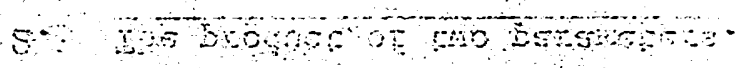

$$
\begin{aligned}
& 3000900
\end{aligned}
$$

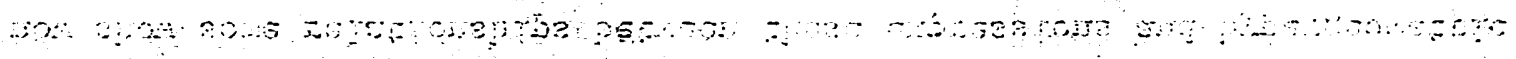

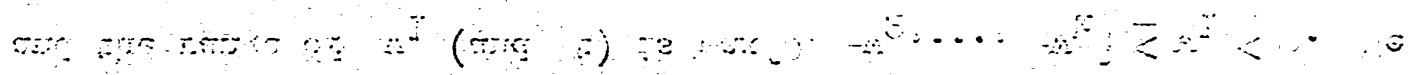

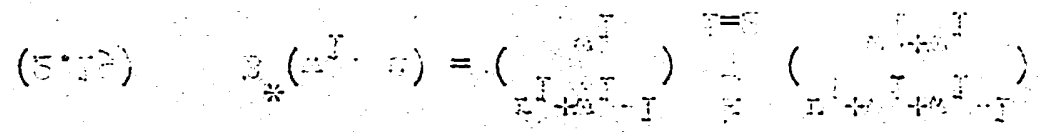

$$
\begin{aligned}
& x=60
\end{aligned}
$$

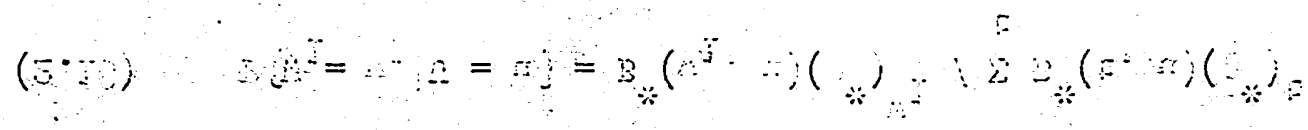


we find

$$
P\left\{x_{1}=x_{1} \mid x_{2}-X_{1}=w\right\}=B^{\prime}\left(x_{1}, w\right) \lambda^{x_{1}} / \sum_{t} B^{\prime}(t, w) \lambda^{t}
$$

where

$$
B^{\prime}\left(x_{1}, w\right)=\left(\begin{array}{c}
r_{1}+x_{1}-1 \\
x_{1}
\end{array}\right)\left(\begin{array}{c}
r_{2}+w+x_{1}-1 \\
w+x_{1}
\end{array}\right)
$$

and where the range of $x_{1}$ (and $t$ ) is $\max (0,-w) \leq x_{1}<\infty$.

It is now straightforward to verify that the conditional distribution can be written

(2.27) $\quad \mathrm{P}\left\{\mathrm{X}_{1} \leq \mathrm{x} \mid \mathrm{X}_{2}-\mathrm{X}_{1}=\mathrm{w}\right\}=\left\{\begin{array}{l}\mathrm{F}_{\mathrm{x}}\left(\mathrm{r}_{1}, \mathrm{r}_{2}+\mathrm{w}, 1+\mathrm{w} ; \lambda\right) / \mathrm{F}\left(\mathrm{r}_{1}, \mathrm{r}_{2}+\mathrm{w}, 1+\mathrm{w} ; \lambda\right) \text { if } \mathrm{w} \geq 0 \\ \mathrm{~F}_{\mathrm{x}+\mathrm{w}}\left(\mathrm{r}_{2}, \mathrm{r}_{1}-\mathrm{w}, 1-\mathrm{w} ; \lambda\right) / \mathrm{F}\left(\mathrm{r}_{2}, \mathrm{r}_{1}-\mathrm{w}, 1-\mathrm{w} ; \lambda\right) \text { if } \mathrm{w}<0\end{array}\right.$ where $x$ is an integer $\geq 0$ if $w \geq 0$; and an integer $\geq-w$ if $w<0$. By analogy with the incomplete ganma and beta functions, we may call the distributions in (2.27) "incomplete hypergeometric functions."

If we define

(2.28) $\alpha=\max (0,-w), \beta=\max (0, w)$

then (2.27) can be put in the alternative form

$$
\begin{aligned}
P\left\{x_{1} \leq x \mid x_{1}-X_{2}=w\right\}= & \frac{F_{x+\alpha}\left(r_{1}+\alpha, r_{2}+\beta, 1+|w| ; \lambda\right)}{F\left(r_{1}+\alpha, r_{2}+\beta, 1+|w| ; \lambda\right)} \\
& x=\alpha, \alpha+1, \ldots, w=0, \pm 1, \pm 2, \ldots .
\end{aligned}
$$

In reliability applications, $\lambda=\mathrm{p}_{1} \mathrm{p}_{2}$ is the reliability of a series system, and the above formulas are relevant. To use the same theory to estimate the reliability $1-q_{1} q_{2}$ of a parallel system we must redefine $x_{1}$ and $x_{2}$ to be the number of failures prior to the $r_{1}^{\text {th }}$ and $r_{2}^{\text {th }}$ successes, and the relevant conditional distribution is 


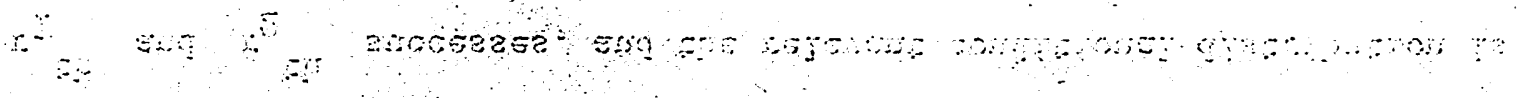

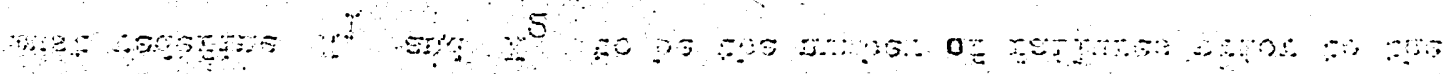

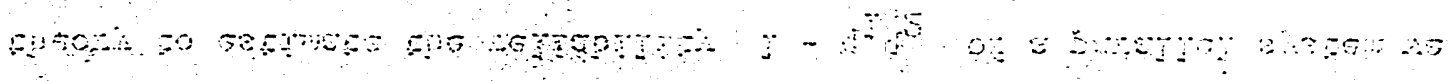

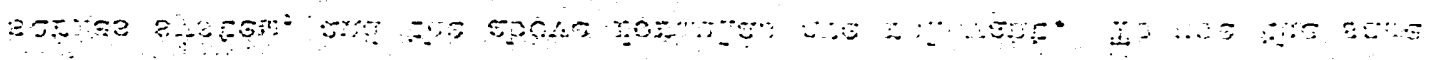

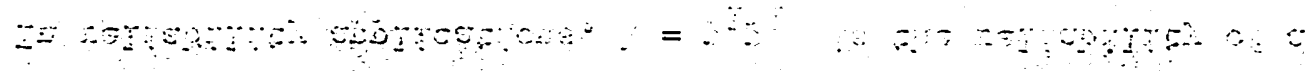

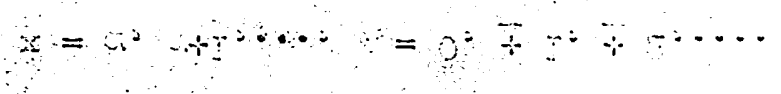

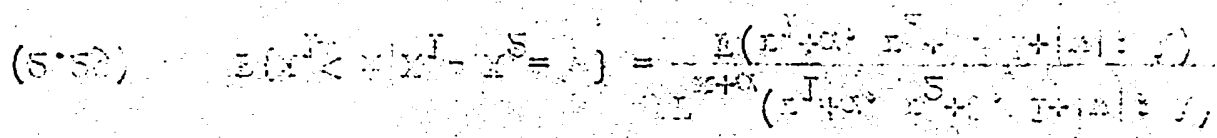

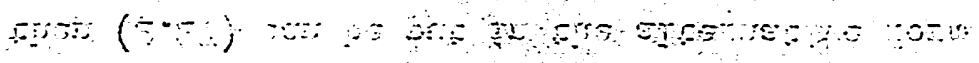

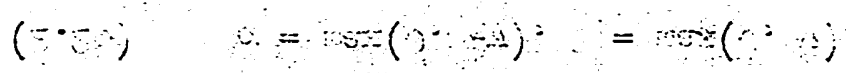

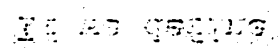

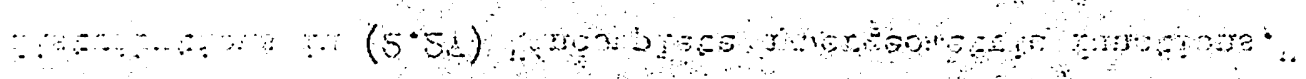

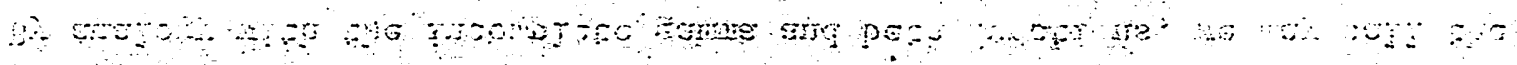
\%o

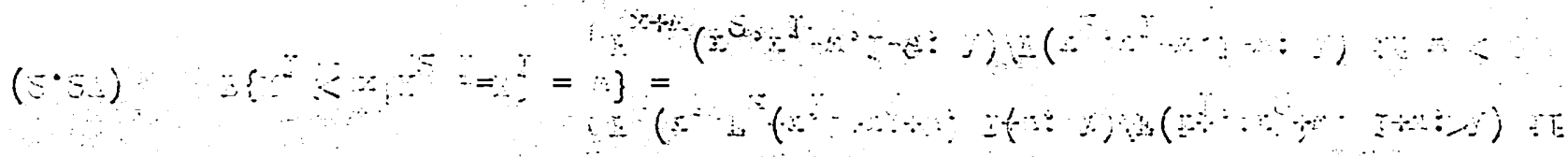

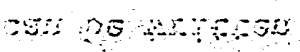

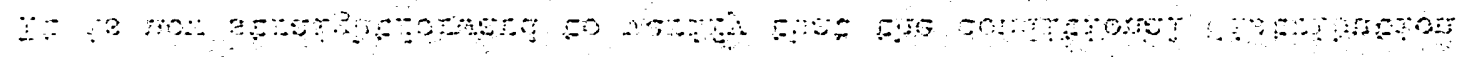

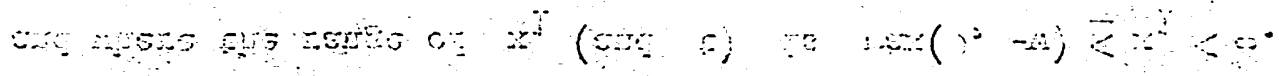

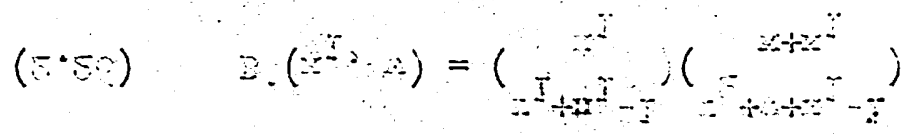
405

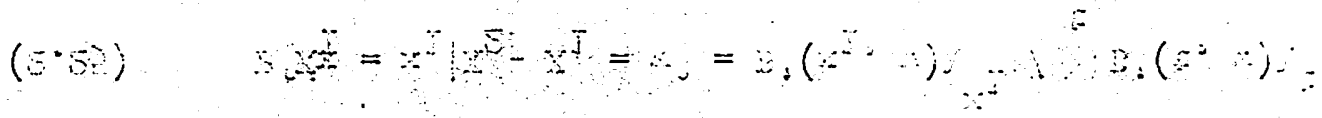
$\therefore \odot$ 


$$
\begin{gathered}
\mathrm{P}\left\{\mathrm{X}_{1}=\mathrm{x}_{1} \mid \mathrm{X}_{2}-\mathrm{x}_{1}=\mathrm{w}\right\}=\frac{\mathrm{f}\left(\mathrm{r}_{1}+\alpha, \mathrm{r}_{2}+\beta, 1+|\mathrm{w}| ; \mu, \mathrm{x}_{1}-\alpha\right)}{\mathrm{F}\left(\mathrm{r}_{1}+\alpha, \mathrm{r}_{2}+\beta, 1+|\mathrm{w}| ; \mu\right)} \\
\mathrm{x}_{1}=\alpha, \alpha+1, \ldots \\
w=0, \pm 1, \pm 2, \ldots
\end{gathered}
$$

where $\alpha$ and $\beta$ are defined in (2.28) and where $\mu=q_{1} q_{2}$. From (2.30) we can obtain confidence intervals for $\mu$ or for the reliability 1 - $\mu$. 2.4 Some special cases.

Suppose $r_{1}=r_{2}=1$, so that $x_{1}$ and $x_{2}$ are the numbers of successes before the first failure in each population. Then $\mathrm{X}_{1}$ and $x_{2}$ have geometric distributions and either from (2.27) or by a more direct argument we find that whether $w \geq 0$ or $w<0$,

$$
P\left\{\min \left(x_{1}, x_{2}\right)=x \mid x_{2}-x_{1}=w\right\}=(1-\lambda) \lambda^{x} \quad x=0,1,2, \ldots
$$

Since the last expression is the same for all w, we conclude that $\min \left(\mathrm{x}_{1}, \mathrm{x}_{2}\right)$ and $\mathrm{x}_{2}-\mathrm{x}_{1}$ are independent1y distributed and that the unconditional distribution of $\min \left(\mathrm{x}_{1}, \mathrm{x}_{2}\right)$ is the geometric distribution given by (2.31). These facts have been noted previously by Ferguson [4].

In this case the upper and lower confidence limits given by (2.13) and (2.14) become

$$
\begin{aligned}
& \lambda_{2}(x)=\sup \left\{\lambda: \sum_{t=0}^{x}(1-\lambda) \lambda^{t} \geq 1-\gamma\right\} \\
& \lambda_{1}(x)=\inf \left\{\lambda: \sum_{t=x}^{\infty}(1-\lambda) \lambda^{t} \leq 1-\gamma\right\}
\end{aligned}
$$

which can be solved explicitly to give

$$
\lambda_{1}=(1-\gamma)^{1 / x}, \lambda_{2}=\gamma^{1 /(x+1)} \text {. }
$$

The lower confidence limit $\lambda_{1}$ is of greater interest in reliability 


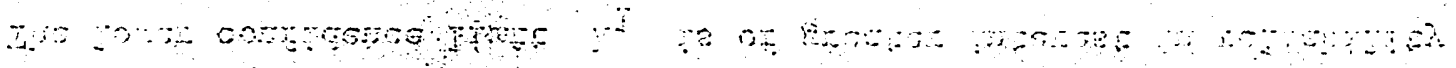

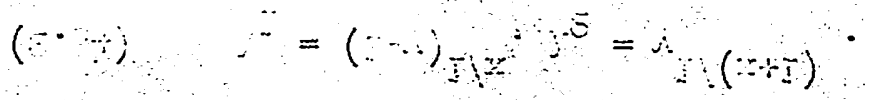

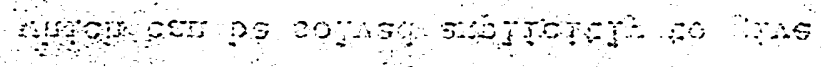

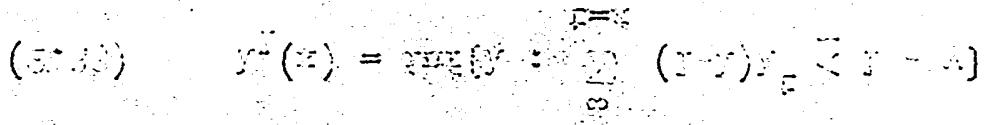

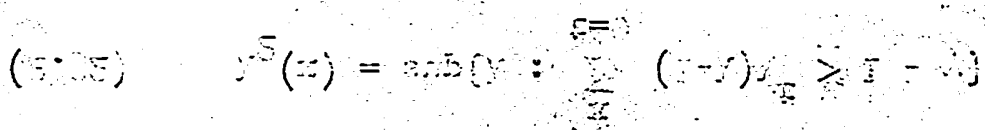

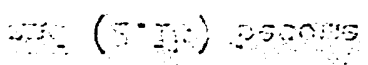

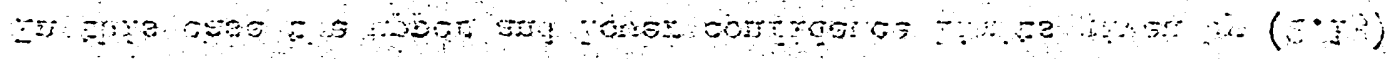

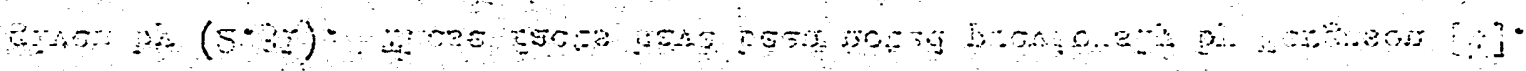

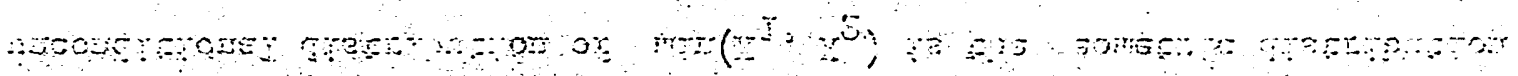

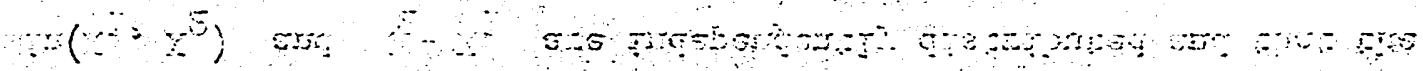
4.00.

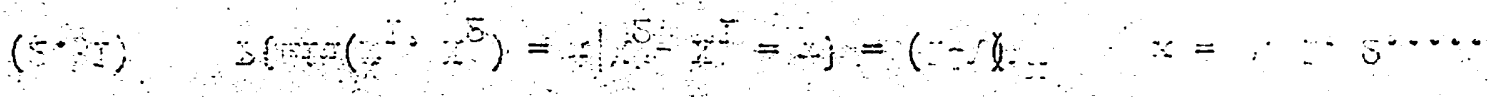

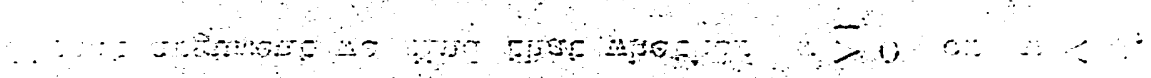

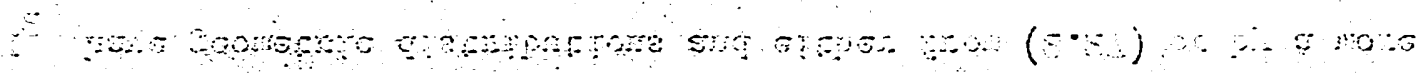

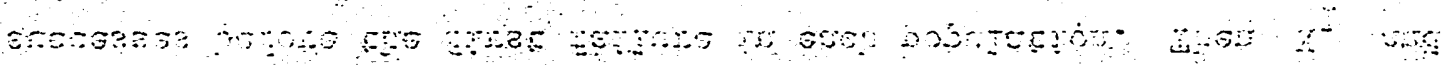

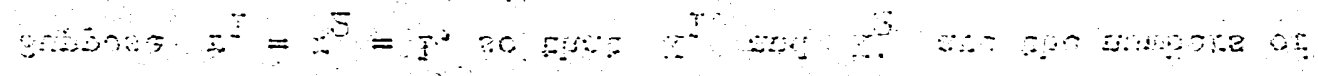

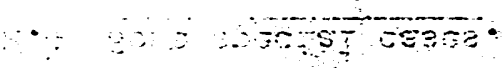

Ho wa opos com

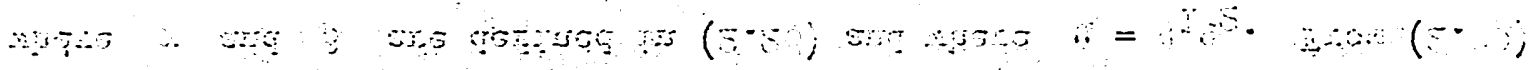

$$
\begin{aligned}
& \because=\because \dddot{\sigma}^{2} \because \Xi^{\cdots}
\end{aligned}
$$

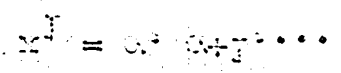

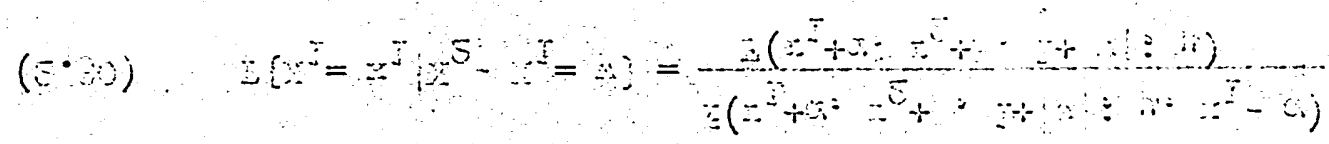


applications, and for large $x, \lambda_{1}$ is asymptotically $1+(1 / x) \log (1-\gamma)$. As an example if $x=100, \alpha=0.05$, then $\lambda_{1}=0.970$.

When $r_{1}=r_{2}=1$, our general method of setting confidence limits amounts to using the unconditional distribution of $\min \left(\mathrm{x}_{1}, \mathrm{x}_{2}\right)$. In reliability applications where we have two dissimilar elements in series with system reliability $\lambda=\mathrm{p}_{1} \mathrm{p}_{2}$, the use of $\min \left(\mathrm{x}_{1}, \mathrm{x}_{2}\right)$ is equivalent to testing individual series systems (rather than components), recording the first system failure, and using that observation to estimate $\mathrm{p}_{1} \mathrm{p}_{2}$ Next suppose $r_{1}$ is arbitrary but $r_{2}=1$. Then if $w=x_{2}-x_{1} \geq 0$,

$$
\mathrm{P}\left\{\mathrm{X}_{1}=\mathrm{x}_{1} \mid \mathrm{x}_{2}-\mathrm{X}_{1}=\mathrm{w}\right\}=\left(\begin{array}{c}
\mathrm{r}_{1}+\mathrm{x}_{1}-1 \\
\mathrm{x}_{1}
\end{array}\right)(1-\lambda)^{\mathrm{r}_{1}{ }_{\lambda} \mathrm{x}_{1}} \mathrm{x}_{1}=0,1,2, \ldots
$$

which is a $\mathrm{NB}\left(\mathrm{r}_{1}, \lambda\right)$ distribution, and is incidentally free of $w$. If $w<0$, then the conditional distribution is a truncated negative binomial,

$$
\begin{array}{r}
P\left\{x_{1}=x_{1} \mid x_{2}-x_{1}=w\right\}=\frac{\left({ }^{r_{1}+x_{1}-1}\right)(1-\lambda)^{r_{1} x_{1}}}{\sum_{t=-w}^{\infty}\left(^{r_{1}+x_{1}-1} t\right)(1-\lambda){ }^{r_{1} t}{ }_{\lambda}} \\
x_{1}=-w_{1},-w_{1}+1,-w_{1}+2, \ldots,
\end{array}
$$

which is not free of $w$. If we define the incomplete beta function as usual by

$$
I_{x}(m, n)=\frac{\Gamma(m+n)}{\Gamma(m) \Gamma(n)} \int_{0}^{x} t^{m-1}(1-t)^{n-1} d t
$$

then the cumulative forms of $(2.35)$ and (2.36) are respectively (see for example [7], [15]),

$$
P\left\{X_{1} \leq x \mid X_{2}-X_{1}=w\right\}=I_{1-\lambda}\left(x_{1}, x+1\right) \quad x=0,1,2, \ldots
$$

and 


\section{$-70$}

suce

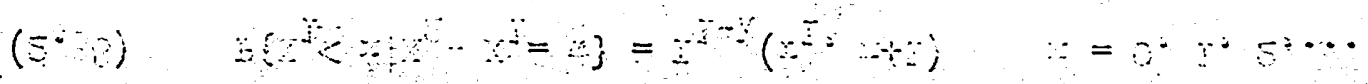
$-0.0019] \cdot 31 \%$

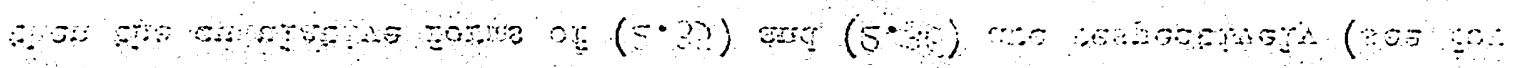

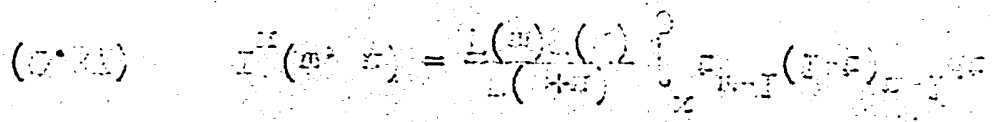

$\because \cdots$

$\therefore$ +

$$
r^{2}=m^{2}+r^{2}+\cdots
$$

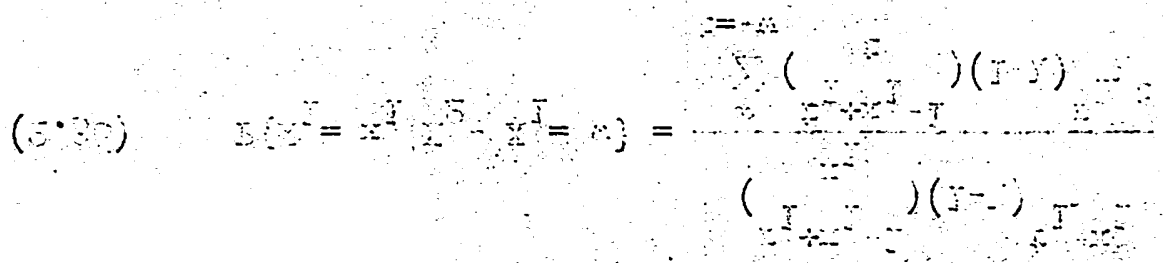

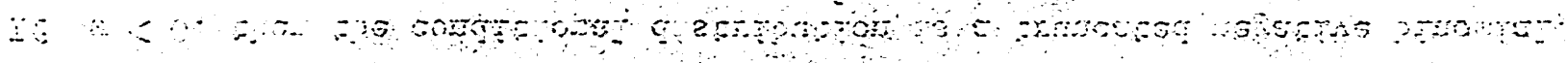

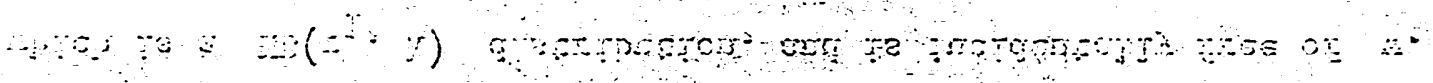

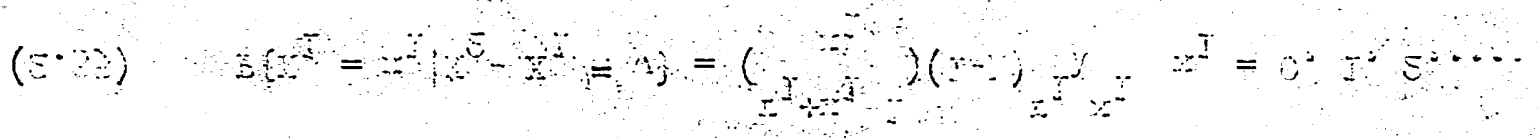

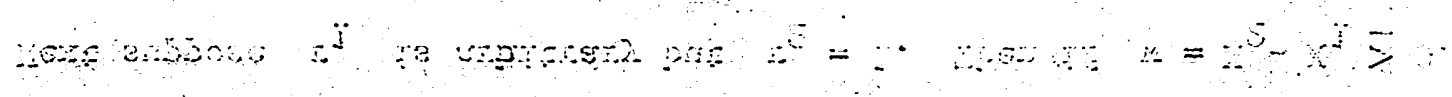
5

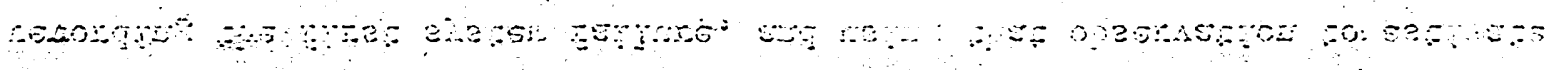

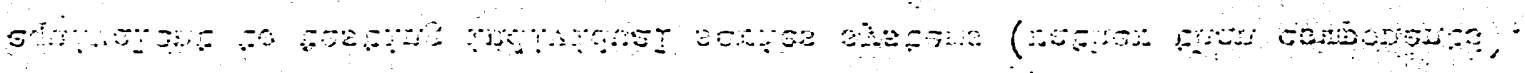

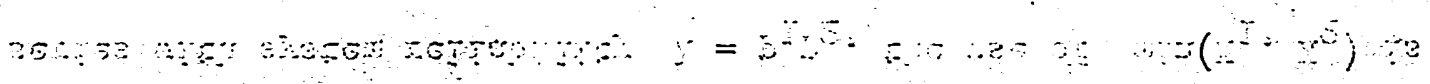

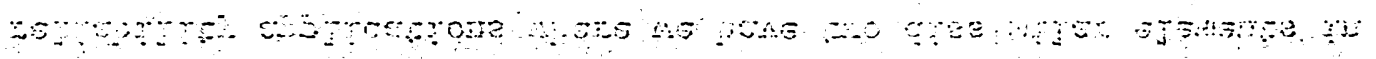

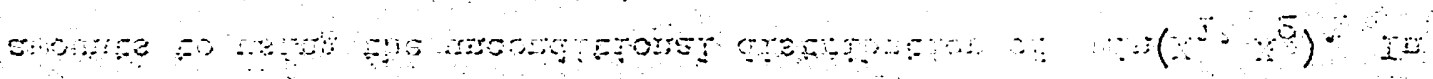

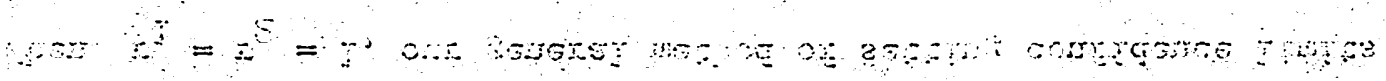
$\because$ w r. c5. 


$$
\begin{gathered}
P\left\{X_{1} \leq x \mid X_{2}-X_{1}=w\right\}=\frac{I_{1-\lambda}\left(r_{1}, x+1\right)-I_{1-\lambda}\left(r_{1},-w\right)}{1-I_{1-\lambda}\left(r_{1},-w\right)} \\
x=-w,-w+1,-w+2, \ldots .
\end{gathered}
$$

Equation (2.38) and other formulas useful for evaluating the negative binomial distribution are considered in more detail in Section 5 below. 2.5 A numerical example.

Table 1 gives a numerical example of lower confidence limits, illustrating the use of the formulas of section 2.3. The point estimates $\underline{\text { Table } 1}$

\begin{tabular}{ccccccl}
\hline Example & $\mathrm{r}_{1}$ & $\mathrm{r}_{2}$ & $\mathrm{x}_{1}$ & $\mathrm{x}_{2}$ & $\begin{array}{l}95 \% \text { lower } \\
\text { confidence } \\
\text { limit for } \\
\mathrm{p}_{1} \mathrm{p}_{2}\end{array}$ & $\begin{array}{l}\text { Point } \\
\text { estimate } \\
\text { of } \mathrm{p}_{1} \mathrm{p}_{2}\end{array}$ \\
\hline $\mathrm{A}$ & 5 & 3 & 45 & 57 & 0.7924 & 0.855 \\
$\mathrm{~B}$ & 10 & 6 & 90 & 114 & 0.8068 & 0.855 \\
\hline
\end{tabular}

for $p_{1} p_{2}$ were calculated from $x_{1} x_{2} /\left(r_{1}+x_{1}\right)\left(r_{2}+x_{2}\right)$. Since Example $B$ is obtained by doubling the data values of Example $A$, the point estimates are the same. Example B has a confidence limit slightly closer to the point estimate, as one would expect. The confidence limits were found by iterating the calculation of the cumlative distribution (2.29) about 5 times, eventually determining the value of $\lambda$ such that (2.29) takes the value 0.95 . This was done on a CDC 6600 computer using a Fortran program and double precision (29 digits). Each iteration required evaluation of the infinite hypergeometric series (2.22). Individual terms were calculated recursively, and the series was arbitrarily declared to have converged as soon as an individual term $a_{n}$ was less than $10^{-10}$. Let 


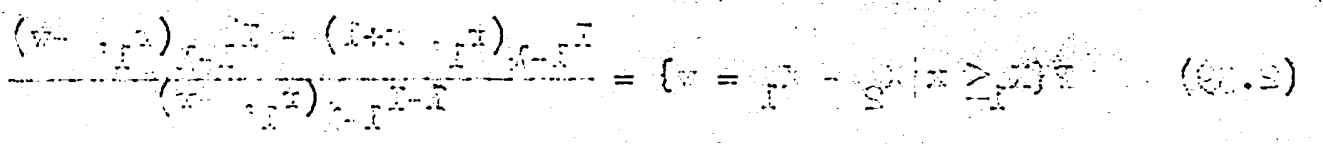

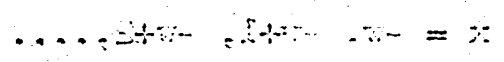

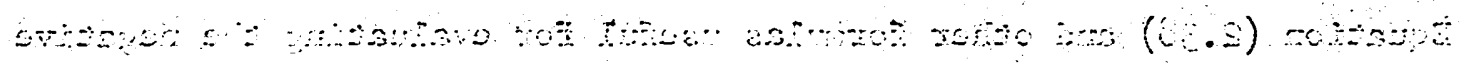

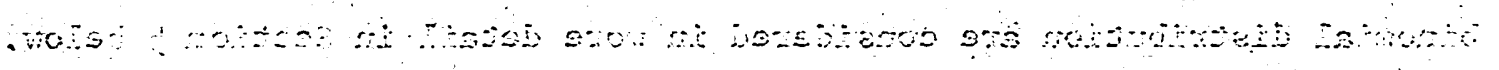

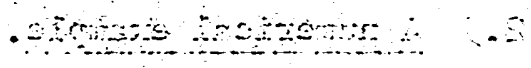

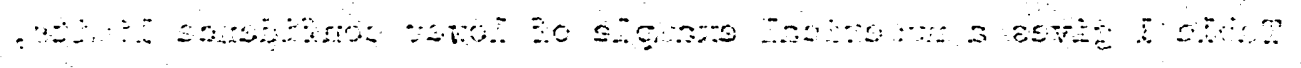

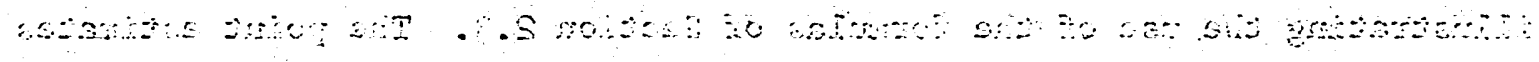

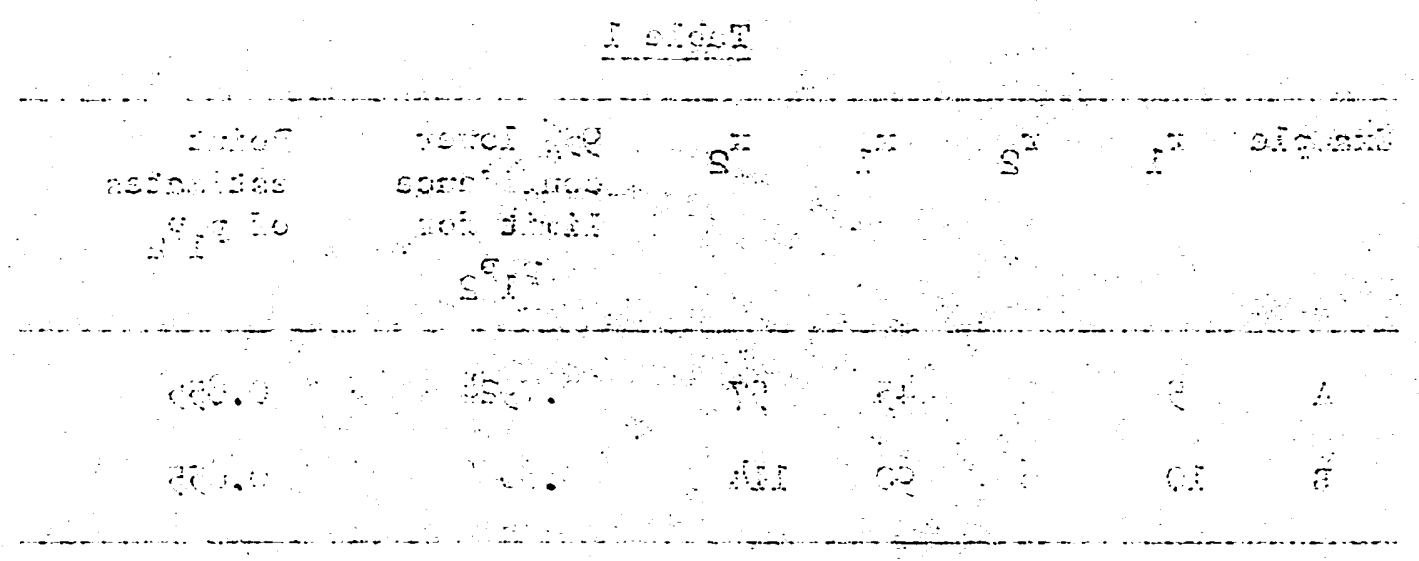

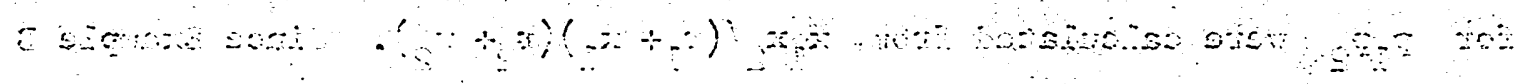

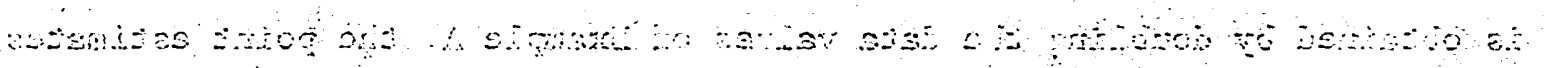

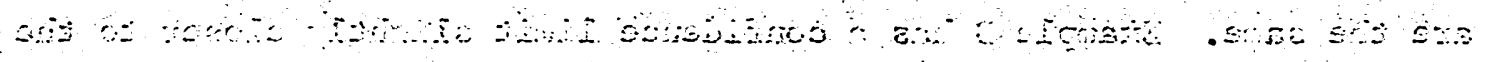

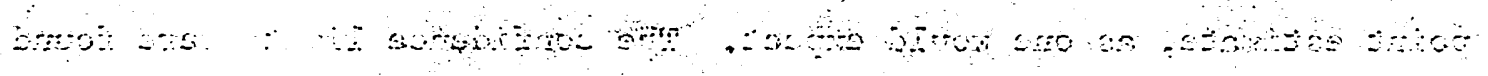

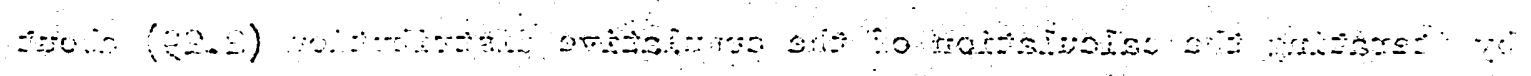

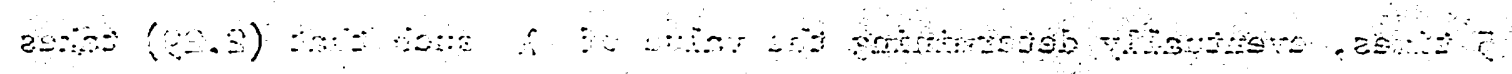

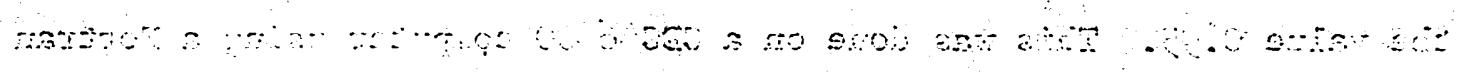

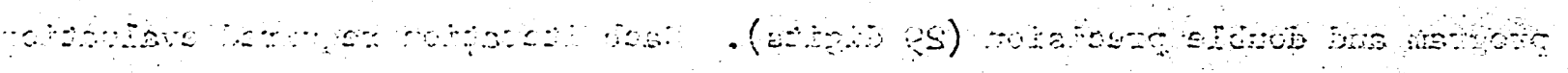

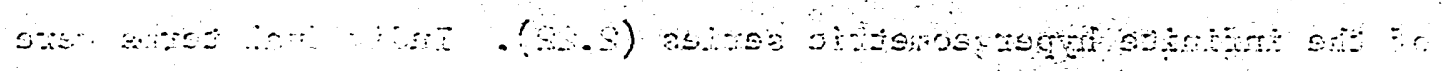

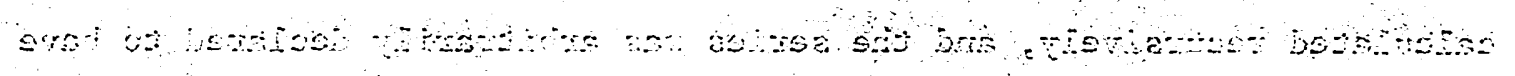

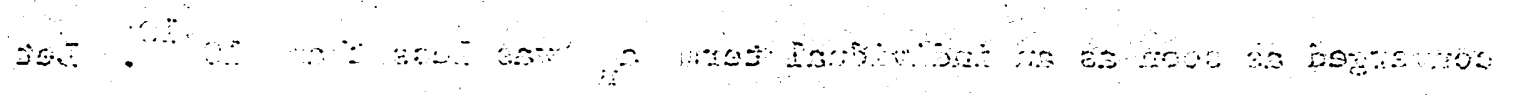


$s_{n}=\sum_{0}^{n} a_{j}$ denote the cumulative sum. In Example $A$, with $\lambda=0.7924$ the calculation stopped with $\mathrm{s}_{201}=16435.77$ and $\mathrm{a}_{201}=8.803 \times 10^{-11}$, and in Example B, with $\lambda=0.8068$ it stopped with $S_{357}=2320684000$ and $a_{357}=8.948 \times 10^{-11}$. Computing time was approximately one second for each iteration.

3. A Method of Mixtures of Distributions for Estimating Other Functions. In Section 2 we have shown how the Iehmann-Scheffé theory can be used to estimate products and quotients of Bernoulli parameters. Occasionally other functions may be of interest. For example, in reliability theory, when a system consists of combinations of series and parallel elements, then the reliability can be expressed as sums and differences of products of Bemoulli parameters. In the present section we will show that sometimes it is possible to tailor the sampling rule to the function to be estimated, and thereby eliminate nuisance parameters and obtain confidence limits.

Lerma 3.1.

Let $p_{1}+q_{1}=p_{2}+q_{2}=1$, and let $\operatorname{Bin}(n, p)$ denote a binomial variate in the usual notation. If $X \sim N B\left(r, q_{1}\right)$ and $(Y \mid X=n) \sim B i n\left(n, P_{2}\right)$, then $Y \sim N B\left(r, q_{1} p_{2} /\left(1-q_{1} q_{2}\right)\right)$.

Proof:

The probability generating function ( $P G F$ ) of $X$ is

$$
\varphi_{X}(t)=\left(\frac{p_{1}}{1-q_{1} t}\right)^{r} .
$$

The PGF of $(Y \mid X=1)$ is

$$
\varphi_{Y \mid X=1}(t)=\left(q_{2}+p_{2} t\right)
$$

By a theorem for the PGF of a random sum (Feller [3], p. 287), the PGF of $\mathrm{Y}$ is 
$\therefore$

30 $(1) \quad \gamma^{5}(5)=\left(y^{5} s\right)$

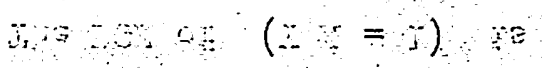

$(\theta) \quad \cdots(c)=\left(\frac{y}{y}\right)=$

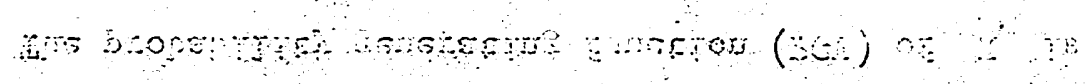

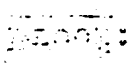

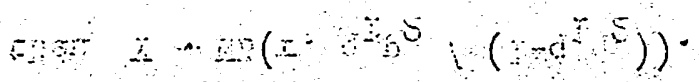

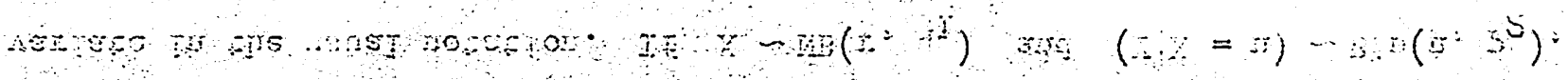

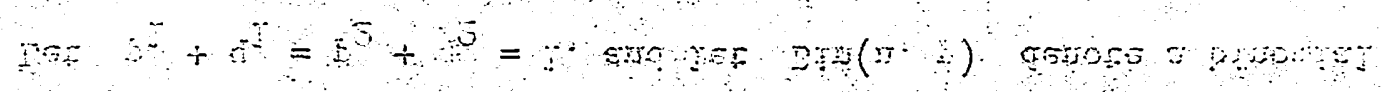
$-7$

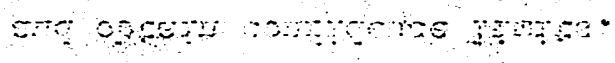

a

4.

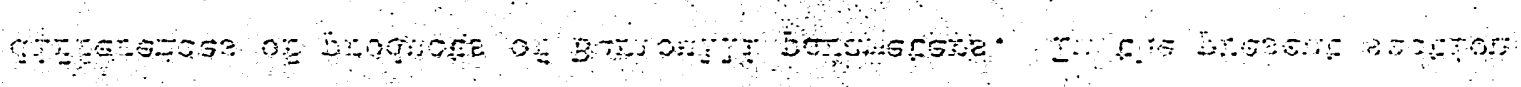

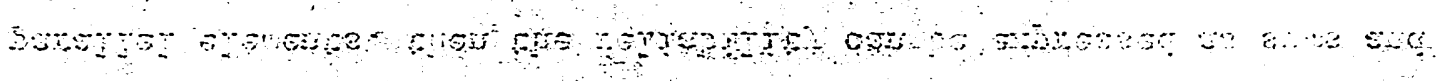

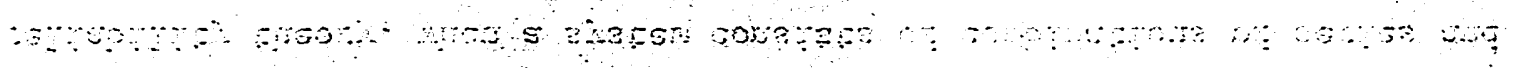

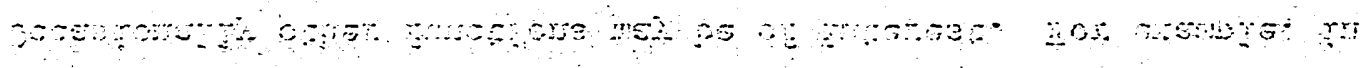

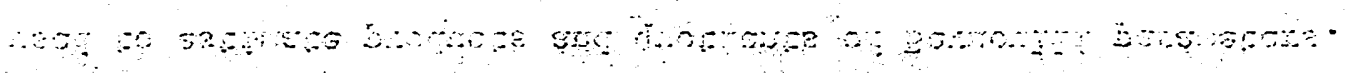

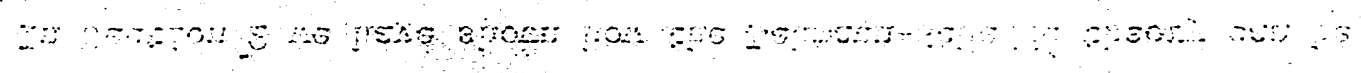

-

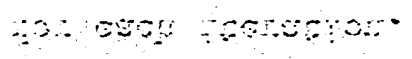

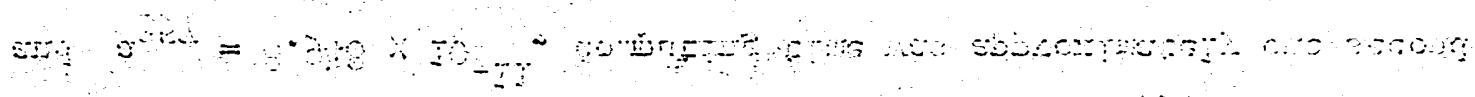

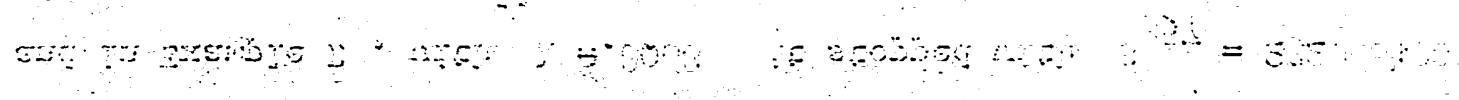

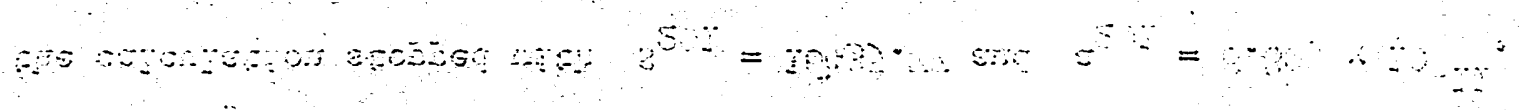

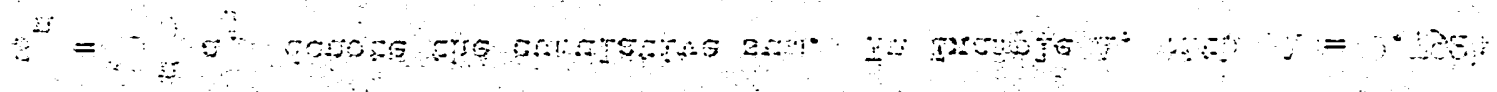




$$
\varphi_{Y}(t)=\varphi_{X}\left(\varphi_{Y \mid X=1}(t)\right)=\left(\frac{p_{1}}{1-q_{1}\left(q_{2}+p_{2} t\right)}\right)^{r}=\left(\frac{q}{1-p t}\right)^{r}
$$

where

$$
\text { (3.4) } \quad q=\frac{p_{1}}{1-q_{1} q_{2}}, p=1-q=\frac{q_{1} p_{2}}{1-q_{1} q_{2}}
$$

which is the PGF of the distribution asserted in the lemma.

Since the marginal distribution of $\mathrm{Y}$ depends only on the parametric function $p$ of $(3.4)$, it is seen that we can make inferences about $p$ by a sampling scheme which first takes a negative binomial sample from population $\pi_{1}$, then a (positive) binomial sample from $\pi_{2}$, where the size of the second sample depends on the outcome of the first sample. We are unable to give an example where the parameters $p$ or $q$ would be of interest. However, by combining the present result with those of section 2, it is possible to make inferences about a wide variety of parametric functions. For example, suppose we have a system with two elements in parallel connected to a third in series. The system reliability is

$$
\text { (3.5) } \quad \theta=\left(1-q_{1} q_{2}\right) p_{3} \text {. }
$$

We can write

$$
\theta=\mathrm{q}_{1} \mathrm{p}_{2} \mathrm{p}_{3} / \mathrm{p},
$$

and since this is of the form (2.3) (with appropriate relabeling of "successes" and "failures" in $\pi_{1}$ ) we can use the method of section 2. In this case we will require the compound sampling described in Lemma 3.1 from populations $\pi_{1}$ and $\pi_{2}$ plus an independent negative binomial sample from each of the populations $\pi_{1}, \pi_{2}, \pi_{3}$. 


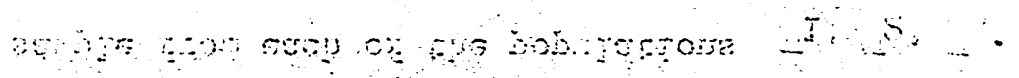

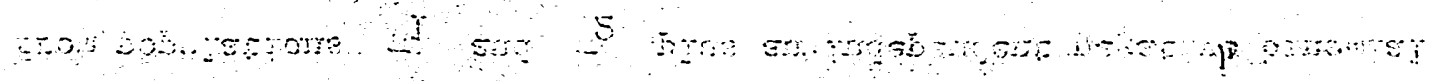

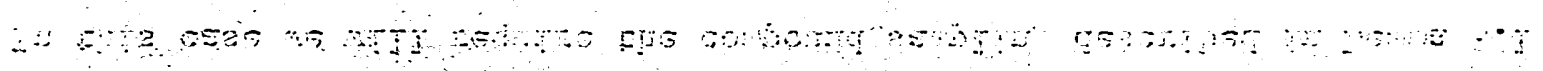
ancesece

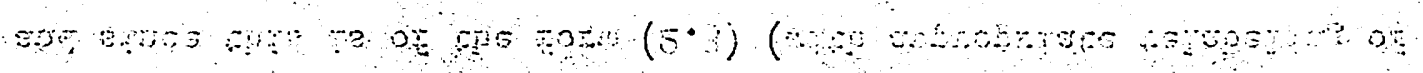
$(\because) \quad=\quad=55$

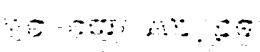

$(-2) \quad \therefore=\left(-y^{5}\right)^{2}$

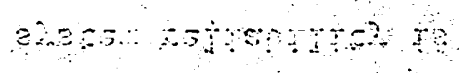

ㅁ. ara b bow

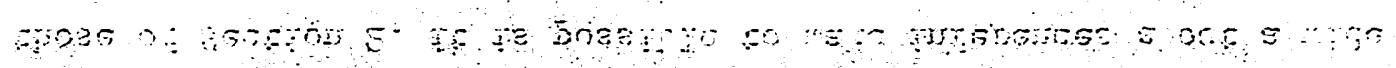

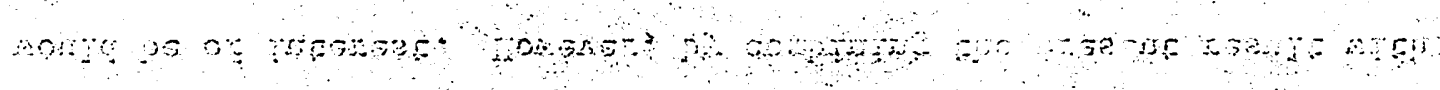
$\therefore 6 \%$ oro bot

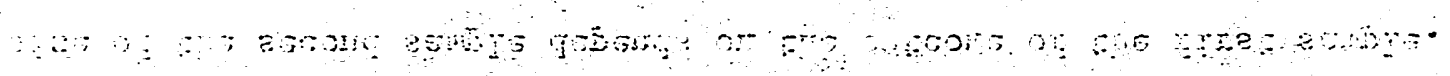

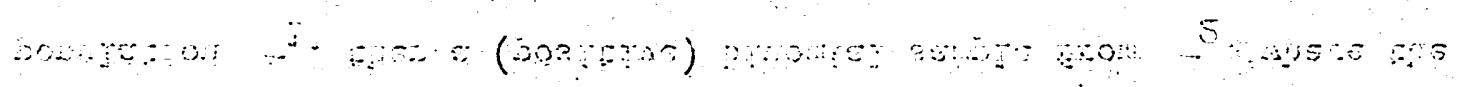

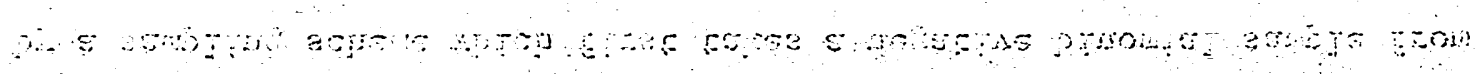

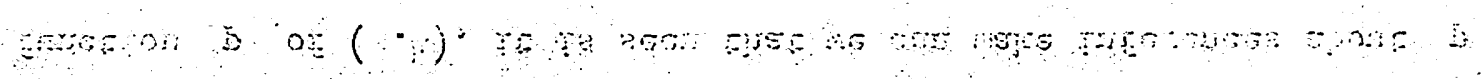

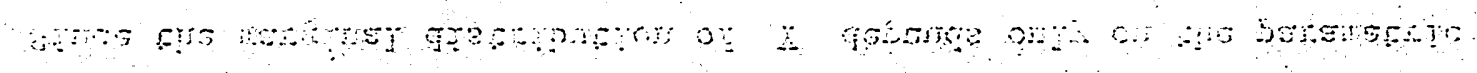

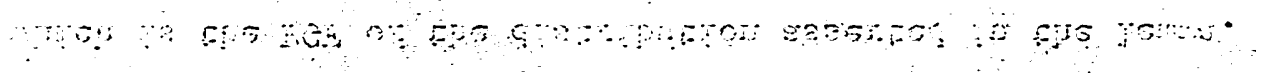

$$
\begin{aligned}
& \left(\because ; \quad=\frac{a^{5}}{b^{5}}=\frac{a^{2}}{n^{5}}\right. \\
& \therefore 35
\end{aligned}
$$

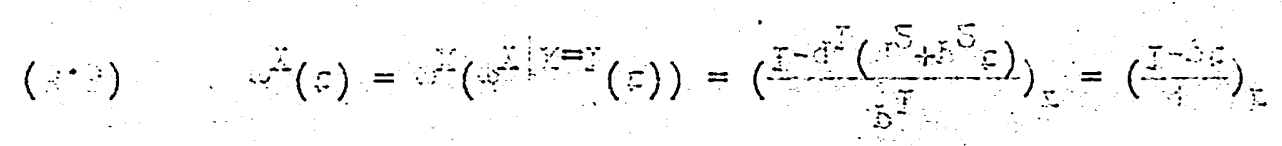


4. Compound Poisson Methods.

In the present section we give some new methods of eliminating nuisance parameters which apply to the estimation of many functions of Bernoulli parameters, in particular sums of products. Unfortunately the methods do have certain deficiencies, as we will indicate. Nevertheless we feel the techniques have mathematical interest and that possibly ways may eventually be found to circumvent or minimize the shortcomings. The following well-known result is the basis of the methods. Lemma 4.1.

$$
\text { If }(X \mid Y=n) \sim \operatorname{Bin}(n, p) \text { and } Y \sim \operatorname{Po}(\lambda)(\text { Poisson with mean } \lambda),
$$

then $\mathrm{X} \sim \mathrm{Po}(\lambda \mathrm{p})$.

Thus we can "compound" binomial variates, converting them to (compound) Poisson variates. If we take a binomial sample whose size is determined randomly by a Poisson variate of known mean $\lambda$, the result is a Poisson variate having mean $\lambda \mathrm{p}$. The purpose of converting to Poisson is to allow later manipulations, but unfortunately the original Poisson observation introduces unwanted variability (see section 4.2 ).

4.1 Horizontal and vertical compounding.

If we independently compound $\operatorname{Bin}\left(n_{i}, p_{i}\right)$ variates by $\operatorname{Po}\left(\lambda_{i}\right)$ variates we get independent $\operatorname{Po}\left(\lambda_{i} p_{i}\right)$ variates. The Lehmann-Scheffé theory we have used in Section 2 applies also to Poisson distributions, and in particular it is possible to obtain conditional distributions depending only on the product of Poisson variates, in this case on $\pi\left(\lambda_{i} p_{i}\right)$. Relevant formulas have been given by Harris [5]. Here $\pi \lambda_{i}$ is known, so we can obtain confidence limits for $\pi_{p_{i}}$. We call the method of this paragraph "horizontal compounding." 


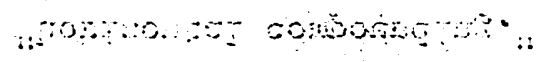

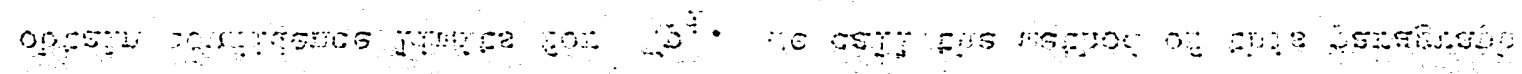

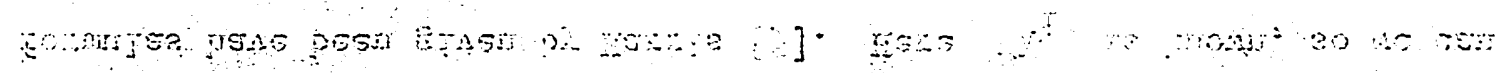

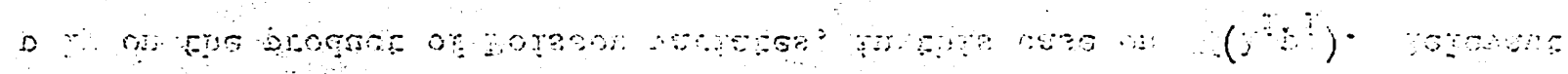

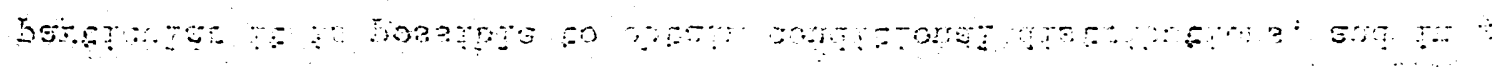

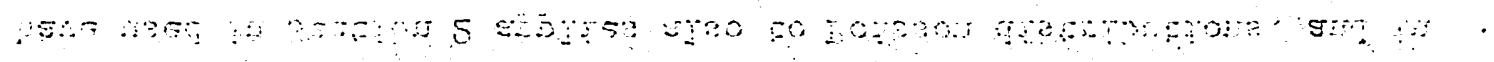

$\because \quad: \quad$ a

$\therefore=$ a

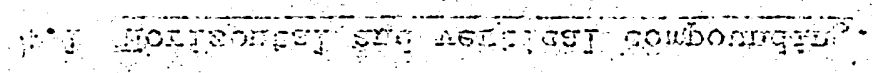

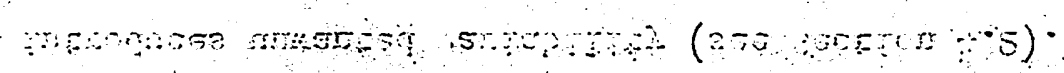

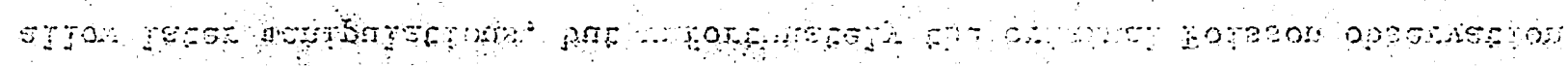

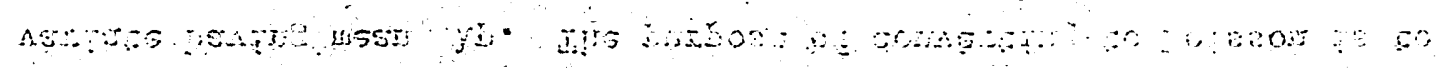

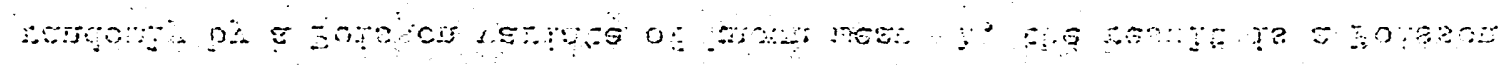

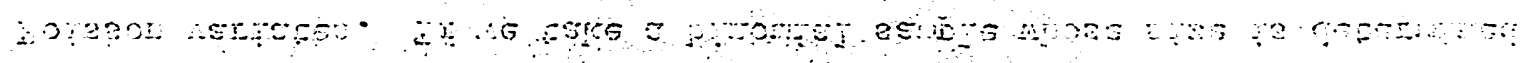

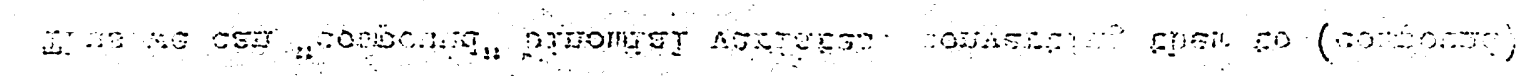

$\sin \because \pi-80(-2)$

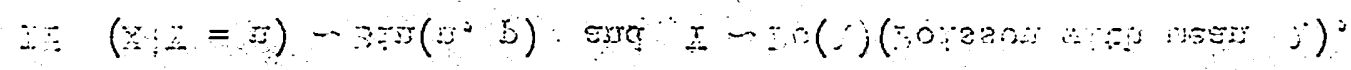

Stene

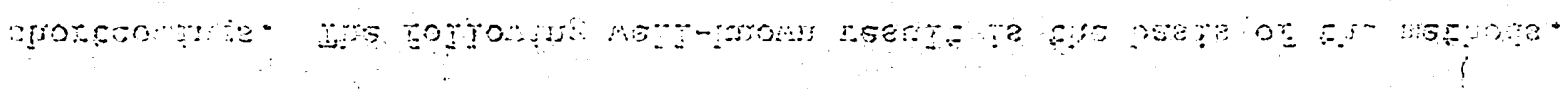

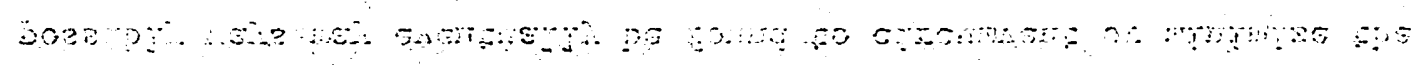

-

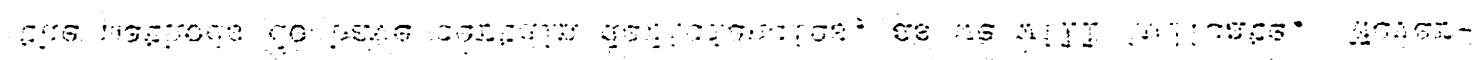

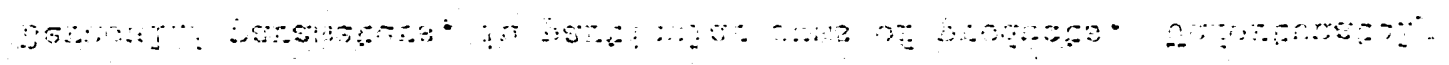

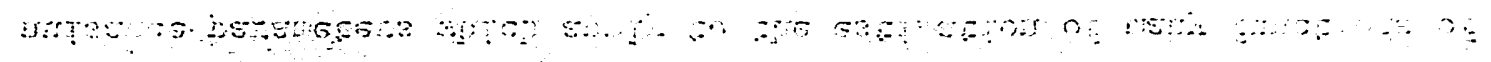

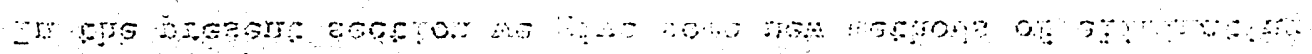

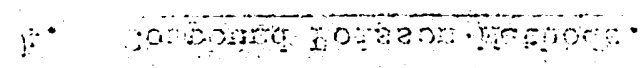


Now suppose $x_{2} \sim \operatorname{Bin}\left(n_{2}, p_{2}\right)$, where $n_{2}$ is an observed value of $x_{1} \sim \operatorname{Bin}\left(n_{1}, p_{1}\right)$, and $n_{1}$ is an observed value of $\operatorname{Po}(\lambda)$. Two applications of Lemma 4.1 give the marginal distribution $x_{2} \sim \operatorname{Po}\left(\lambda p_{1} p_{2}\right)$. This "vertical compounding" can clearly be carried to any number of steps. Since $\lambda$ is known, we need only tables of the Poisson distribution to find confidence limits for $\pi p_{i}$.

To estimate sums of products of Bernoulli parameters one can appeal to the reproductive property of the Poisson distributions, and possibly also to the following lemma (Feller [3], p. 301, problem 3):

Lemma 4.2 .

In $Y$ Bernoulli trials, where $Y \sim P o(\lambda)$, the numbers of successes and failures are stochastically independent.

For example, $\theta$ in (3.5) can be rearranged to $\theta=p_{3}\left(p_{1}+q_{1} p_{2}\right)$. This suggests observing $Y=n$ from $P \circ(\lambda), X_{1}=x_{1}$ from $\operatorname{Bin}\left(n, p_{1}\right), X_{2}=x_{2}$ from $\operatorname{Bin}\left(n-x_{1}, p_{2}\right)$. Then $x_{1} \sim \operatorname{Po}\left(\lambda p_{1}\right)$ and $x_{2} \sim \operatorname{Po}\left(\lambda q_{1} p_{2}\right)$ and $x_{1}$ and $x_{2}$ are independent. Thus $x_{1}+x_{2} \sim P o\left(\lambda p_{1}+\lambda q_{1} p_{2}\right)$, and taking $\mathrm{x}_{3}$ from $\operatorname{Bin}\left(\mathrm{x}_{1}+\mathrm{x}_{2}, \mathrm{p}_{3}\right)$ gives the desired unconditional distribution $\mathrm{x}_{3} \sim \mathrm{Po}\left(\lambda \mathrm{p}_{3}\left(\mathrm{p}_{1}+\mathrm{q}_{1} \mathrm{p}_{2}\right)\right)$.

It is clear that there are many ways to estimate sums of products by horizontal and vertical compounding. For expressions involving differences rather than sums, algebraic manipulations using the identities $p_{i}+q_{i}=1$ can be used to reverse the signs. Hwang [6] has classified functions which can be estimated by these various techniques. 4.2 Critique.

In either horizontal or vertical compounding, the use of the variates $\mathrm{Y}$ having known Poisson distribution is rightly viewed with suspicion. 


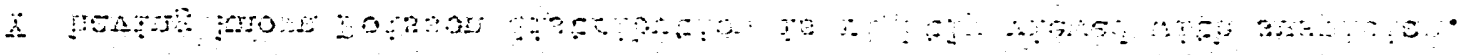

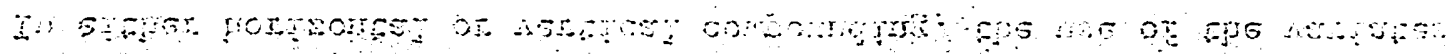

$\because 306$

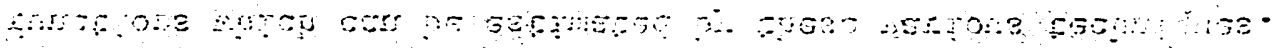
$b^{\ddot{4}}+4=\because$ a

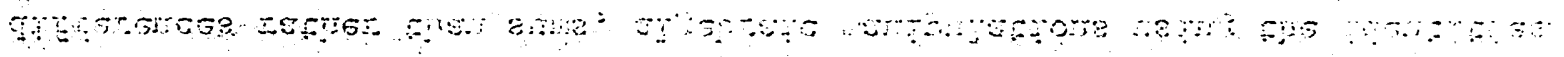

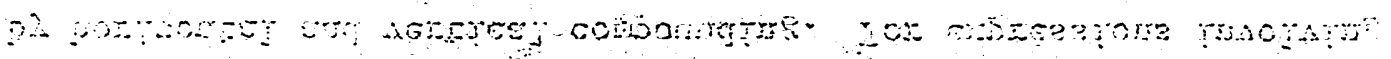

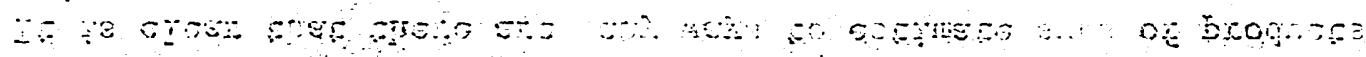
$x^{2}-\operatorname{sos}(2+4)$

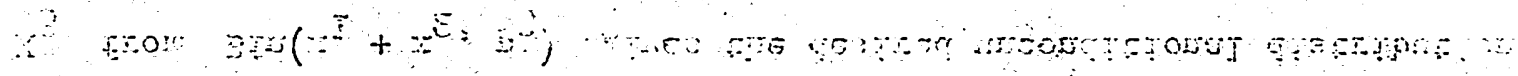

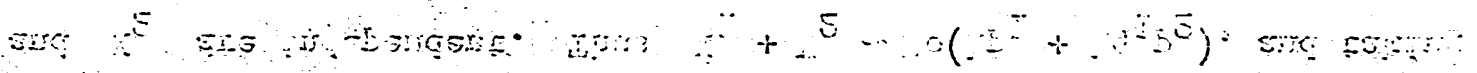

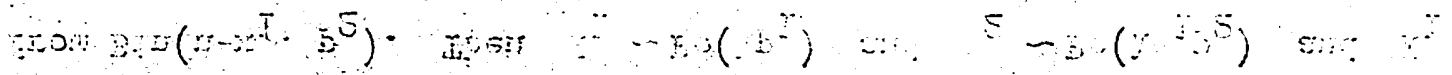

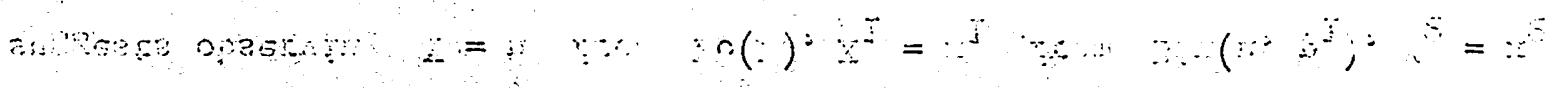

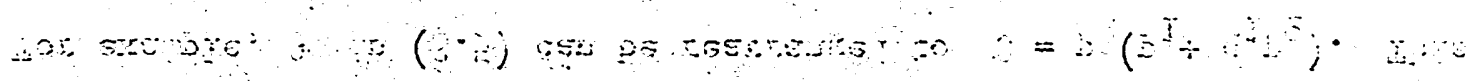

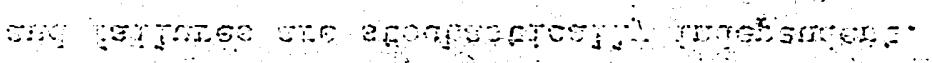

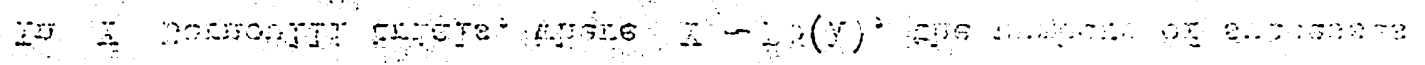
30

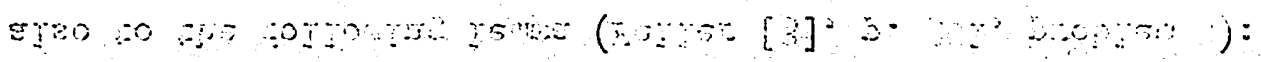

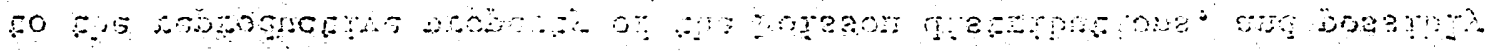

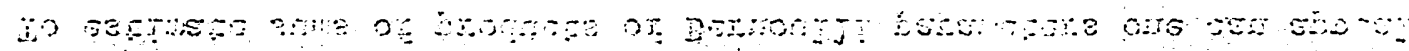
$\because \operatorname{sing}$

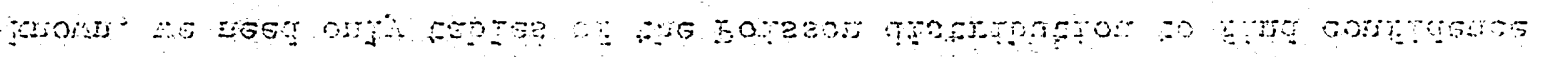

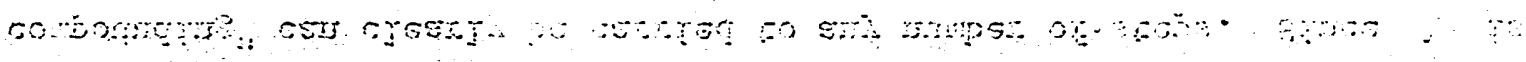

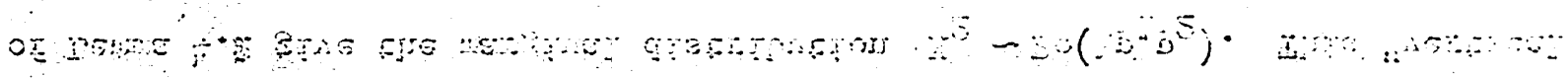

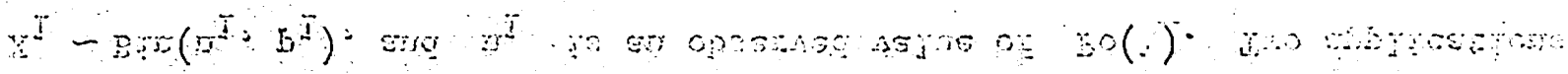

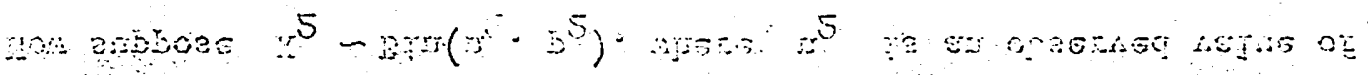


Technically, $\mathrm{Y}$ is an ancillary statistic since its distribution does not depend on the unknown parameters. Contrary to our suggested procedures, either Bayesian theory or the likelihood principle would demand inferences conditional on the observed value of $Y$.

The variates $Y$ do indeed introduce undesirable variability, which is the price we pay for elimination of nuisance parameters. We can get some idea of the effect of the added variability by considering a simplified case. Take $\mathrm{Y} \sim \mathrm{Po}(\lambda)$ and $(\mathrm{X} \mid \mathrm{Y}=\mathrm{n}) \sim \operatorname{Bin}(\mathrm{n}, \mathrm{p})$. We may compare the conditional (given $\mathrm{Y}=\mathrm{n}$ ) point estimator

(4.1) $\quad \hat{\mathrm{p}}=\mathrm{x} / \mathrm{n}$

with the unconditional estimator

(4.2) $\quad \widetilde{p}=x / \lambda$.

The simplest comparison is that of conditional and unconditional variances:

(4.3) $\quad \operatorname{Var}(\hat{\mathrm{p}} \cdot \mathrm{Y}=\mathrm{n})=\mathrm{pq} / \mathrm{n} ; \operatorname{Var} \tilde{\mathrm{p}}=\mathrm{p} / \lambda$

A measure of the efficiency of $\tilde{p}$ relative to $\hat{p}$ is the quotient $(\mathrm{pq} / \mathrm{n}) /(\mathrm{p} / \lambda)=\mathrm{q} \lambda / \mathrm{n}$, in which the relevant factor is $\mathrm{q}$, since $\lambda / \mathrm{n}$ is of the order of unity for large $\lambda$. We conclude that the additional variability introduced by $\mathrm{Y}$ is very serious when $\mathrm{q}$ is sma11, but not serious when $p$ is small so that $q$ is close to 1 . On the other hand when $p$ is small, it becomes necessary to choose $\lambda$ to compromise between large samples ( $\lambda$ large) and a high "discreteness index" ( $\lambda$ p small; see Section 1.2 ).

In any compounding scheme it would be advisable to consider questions of efficiency and "discreteness index" at each stage. 


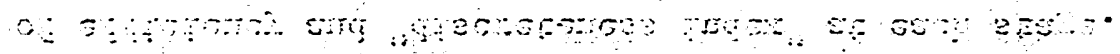

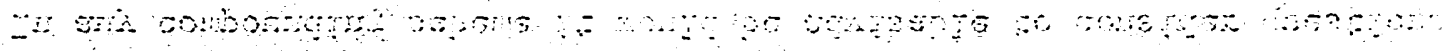

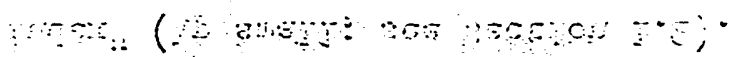

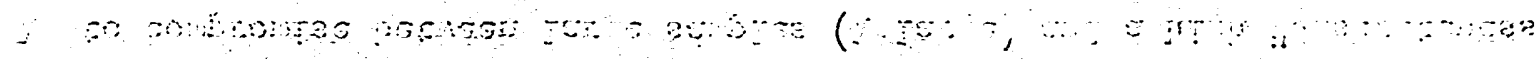

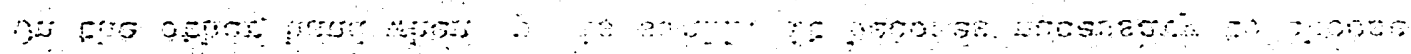

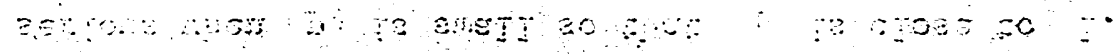

A.

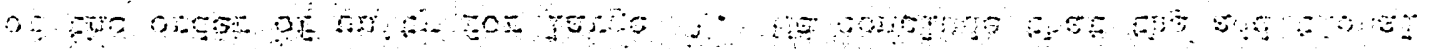

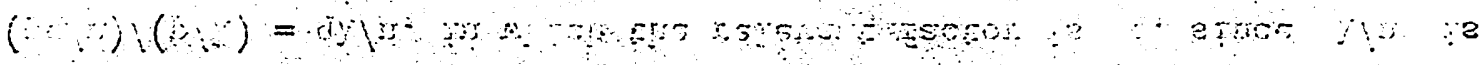

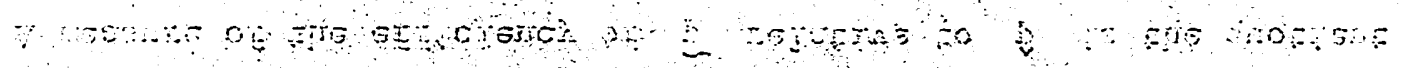

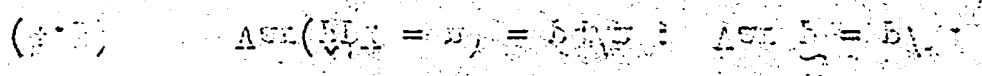

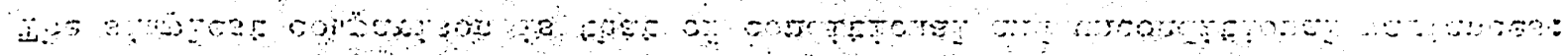

$(\div) \quad-\quad=3$

Met a

$(\because \cdots) \quad \stackrel{\cdots}{*}=\cdots$

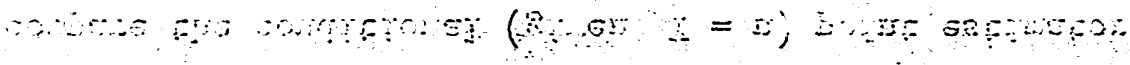

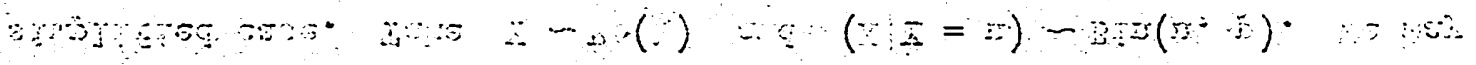

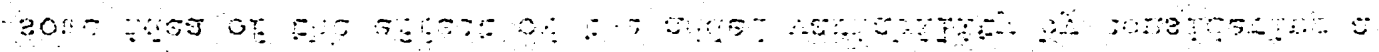

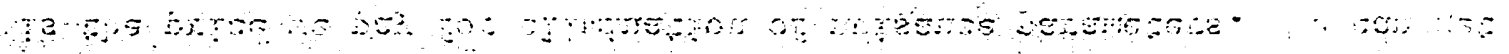

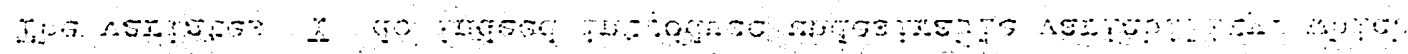

on a

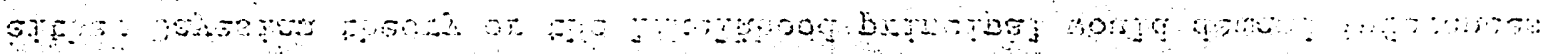

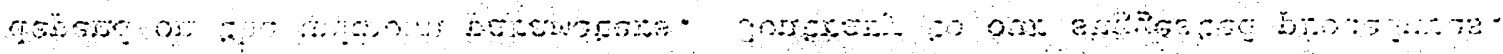

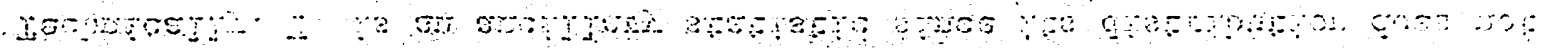




\section{Estimation of Products Using Geometric Variates.}

A sequence of Bernoulli trials--that is, a Bernoulli process--gives a record of "successes" and "failures," which may conveniently be recorded as zeros and ones. Let $\mathrm{p}=\mathrm{P}$ (success) $=\mathrm{P}(0), \mathrm{q}=1-\mathrm{p}=\mathrm{P}$ (failure) $=\mathrm{P}(1)$. In a record such as 0111010001101 the information about $p$ is traditionally summarized by $" \mathrm{X} \sim \operatorname{Bin}(13, \mathrm{p})$ and $\mathrm{X}=6$," if it was decided in advance to stop with the thirteenth observation, or by " $\mathrm{X} \sim \mathrm{NB}(7, \mathrm{p})$ and $\mathrm{X}=6$ " if it was decided in advance to stop with the seventh failure. In the latter case we may alternatively report the observation of seven independent geometric variates: $\left(\mathrm{x}_{1}, \ldots, \mathrm{x}_{7}\right)=(1,0,0,1,3,0,1)$ where $\mathrm{x}_{i}$ is the number of successes between the $(i-1)^{\text {st }}$ and $i^{\text {th }}$ failures. We write $x_{i} \sim \operatorname{Geom}(p) \quad$ where

(5.1) $\quad X \sim \operatorname{Geom}(p)$ means $P(X=x)=(1-p) p^{X}$ for $x=0,1,2, \ldots$

In the present section we exploit properties of geometric variates to estimate products of Bernoulli parameters. Our methods are discrete analogs of those proposed by Lieberman and Ross [11] for the estimation of sums of exponential parameters.

5.1 A method for estimating $\mathrm{p}_{1} \underline{\mathrm{P}}_{2}$ and its theoretical basis.

The following easily verified lemma follows from Ferguson's characterization of the geometric distribution [4].

Lemma 5.1.

Let $X$ and $Y$ be independent, $X \sim \operatorname{Geom}\left(p_{1}\right), Y \sim \operatorname{Geom}\left(p_{2}\right)$, and let $q_{1}=1-p_{1}, q_{2}=1-p_{2}$,

(5.2) $\quad U=\min (X, Y), \quad V=Y-X$.

Then $U$ and $V$ are independent, $U \sim \operatorname{Geom}\left(p_{1} p_{2}\right)$, and 


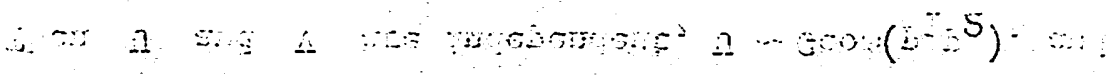

$(-5) \quad n=\operatorname{lng}(\because 2)$

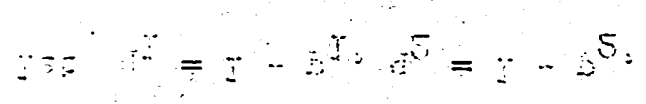

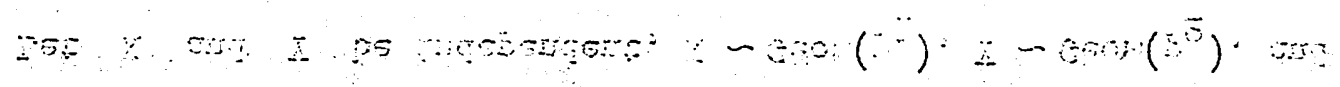

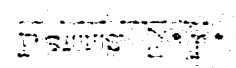

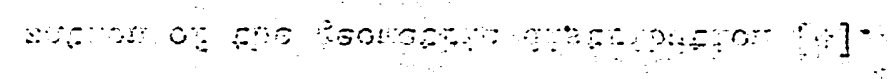

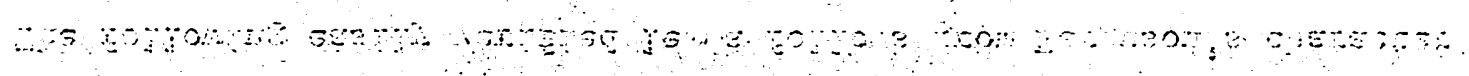

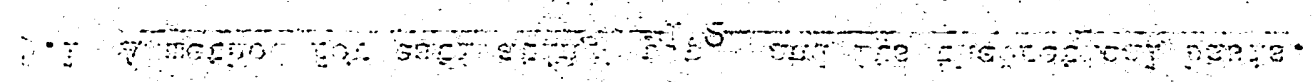

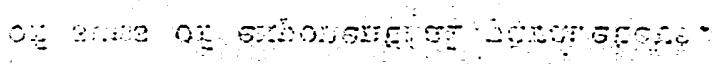

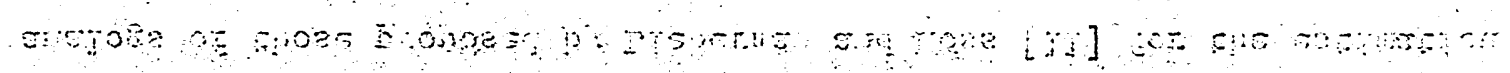

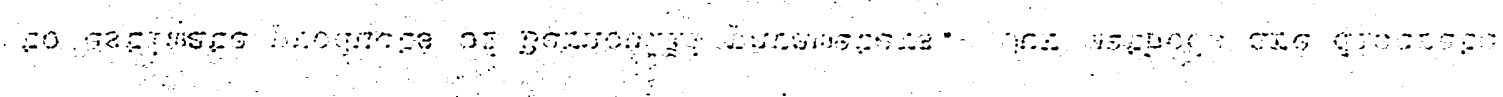

-

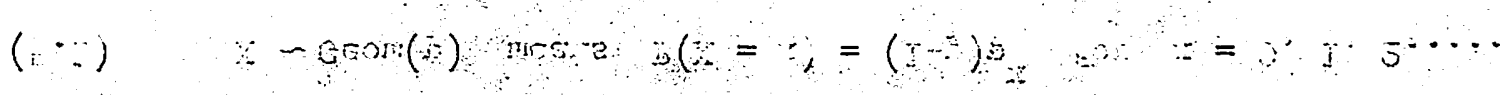

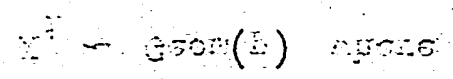

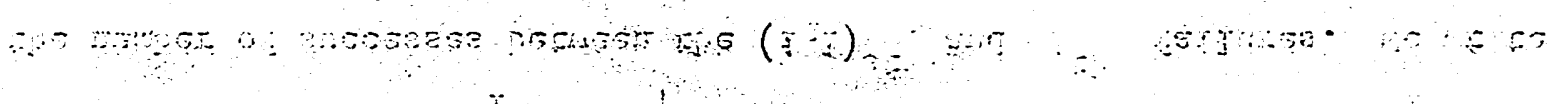

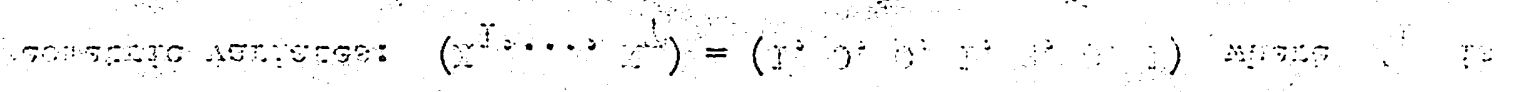

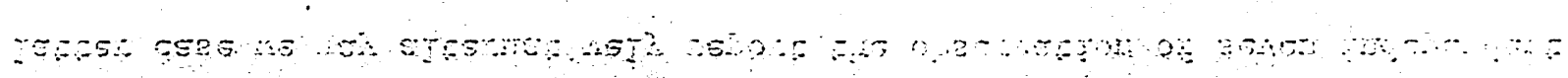

o to

50 as *

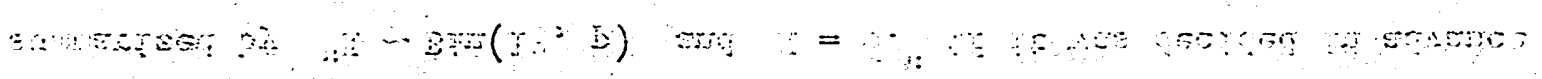

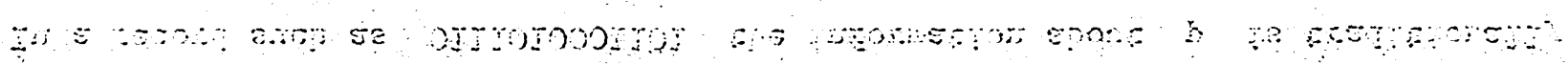

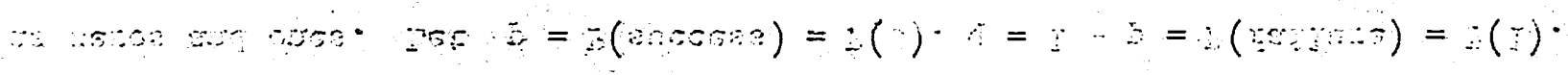

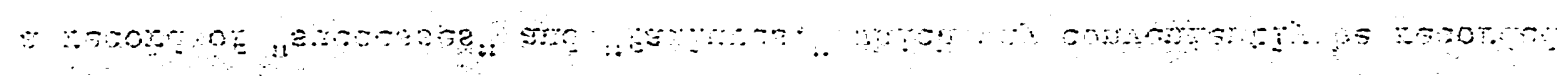

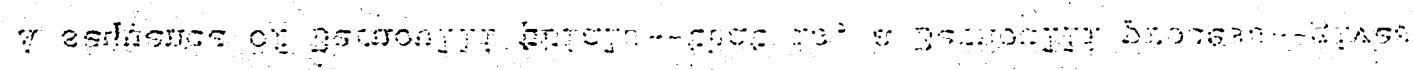

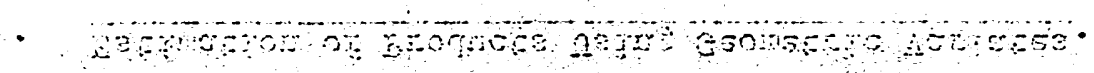


(5.3) $\quad P\{v=v\}=\left\{\begin{array}{ll}c p_{2}^{v} & v>0 \\ c p_{1}^{-v} & v \leq 0\end{array} \quad\right.$ where $c=q_{1} q_{2} /\left(1-p_{1} p_{2}\right)$

Since the distribution of $U$ depends only on $\mathrm{p}_{1} \mathrm{p}_{2}$, one or more U-values could be used to estimate $\mathrm{p}_{1} \mathrm{P}_{2}$. However, there is "left over" information in the values of $\mathrm{V}$ which can be used for increased efficiency.

The variate $V$ is rather like a $\operatorname{Geom}\left(\mathrm{p}_{2}\right)$ variate when $\mathrm{V}>0$ and $-V$ is like $\operatorname{Geom}\left(\mathrm{P}_{1}\right)$ when $\mathrm{V}<0$. The case $\mathrm{V}=0$ in fact presents a difficulty (which did not arise in the Lieberman-Ross continuous mode1) which we overcome by an asymmetric treatment, arbitrarily classifying $\mathrm{V}=0$ with the negative values. From (5.3) we have:

Lemma 5.2 .

$(\mathrm{V}-1 \mid \mathrm{V}>0) \sim \operatorname{Geom}\left(\mathrm{p}_{2}\right) . \quad(-\mathrm{V} \mid \mathrm{V} \leq 0) \sim \operatorname{Geom}\left(\mathrm{p}_{1}\right)$

Now let us suppose $\mathrm{x}_{1}, \ldots, \mathrm{x}_{\mathrm{m}}$ are independent $\operatorname{Geom}\left(\mathrm{p}_{1}\right)$ variates and $Y_{1}, \ldots, Y_{n}$ are independent $\operatorname{Geom}\left(\mathrm{P}_{2}\right)$ variates. Let $U_{1}=\min \left(X_{1}, Y_{1}\right)$, $\mathrm{V}_{1}=\mathrm{Y}_{1}-\mathrm{X}_{1}$. We now use $\mathrm{V}_{1}$ to construct an observation $\mathrm{U}_{2}$, independent of $U_{1}$ and having the same $\operatorname{Geom}\left(\mathrm{p}_{1} \mathrm{p}_{2}\right)$ distribution.

Let us define

(5.4) $\quad \mathrm{U}_{2}= \begin{cases}\min \left(\mathrm{x}_{2}, \mathrm{v}_{1}-1\right) & \text { if } \mathrm{v}_{1}>0 \\ \min \left(-\mathrm{v}_{1}, \mathrm{Y}_{2}\right) & \text { if } \mathrm{v}_{1} \leq 0\end{cases}$

(5.5) $\quad \mathrm{v}_{2}= \begin{cases}\mathrm{v}_{1}-1-\mathrm{x}_{2} & \text { if } \mathrm{v}_{1}>0 \\ \mathrm{y}_{2}+\mathrm{v}_{1} & \text { if } \mathrm{v}_{1} \leq 0 .\end{cases}$

From Lemmas 5.1 and 5.2 we have:

Lemma 5.3.

(i) $\mathrm{U}_{1}, \mathrm{U}_{2}$ and $\mathrm{V}_{2}$ are mutually independent; (ii) $\mathrm{U}_{2} \sim \operatorname{Geom}\left(\mathrm{p}_{1} \mathrm{p}_{2}\right)$; (iii) $v_{2}$ has the distribution (5.3). 


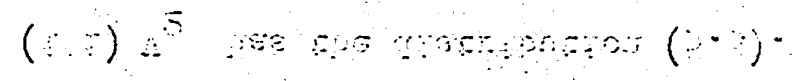

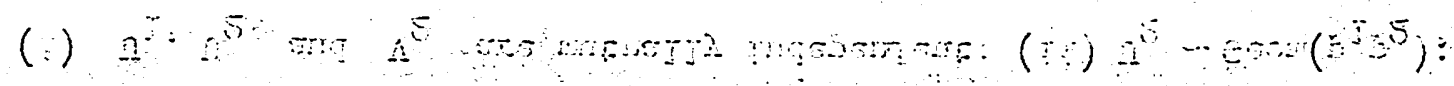

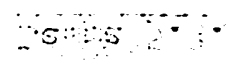

an mate

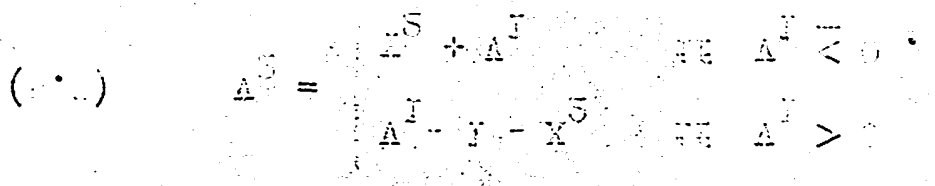

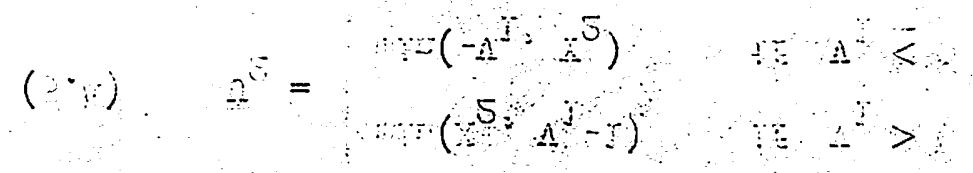

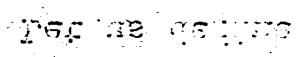

$\because a^{2}$ ar ano

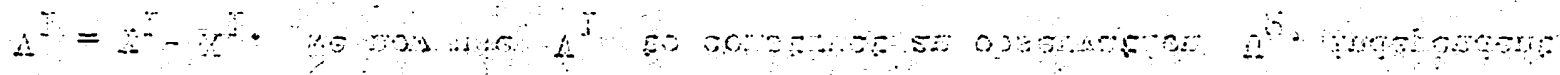

-

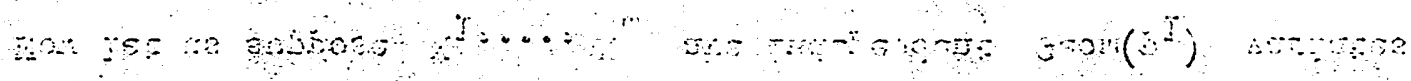

$(A H>0)-\cos \left(0^{5}\right) \cdot(-4 \Lambda<0)-\theta \cos (t)$

$\because 5$

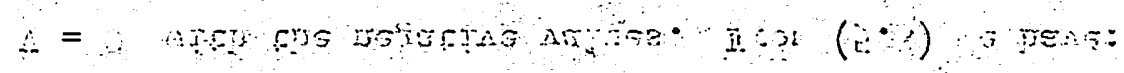

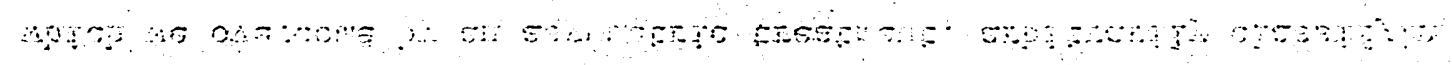

$\therefore$.

का

H. A

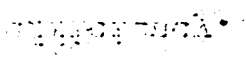

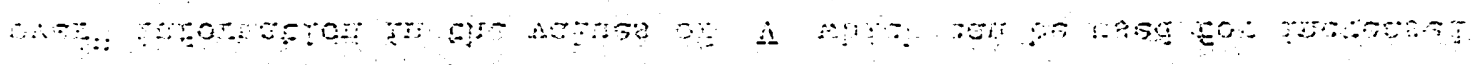

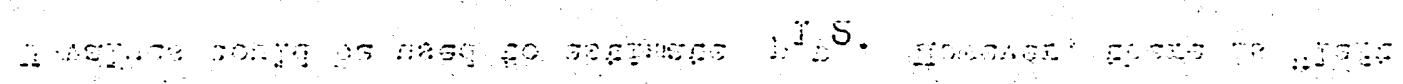

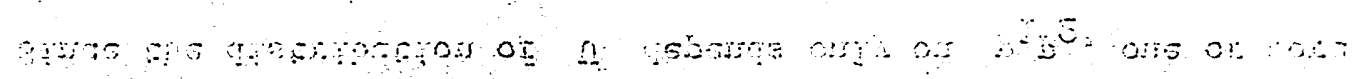

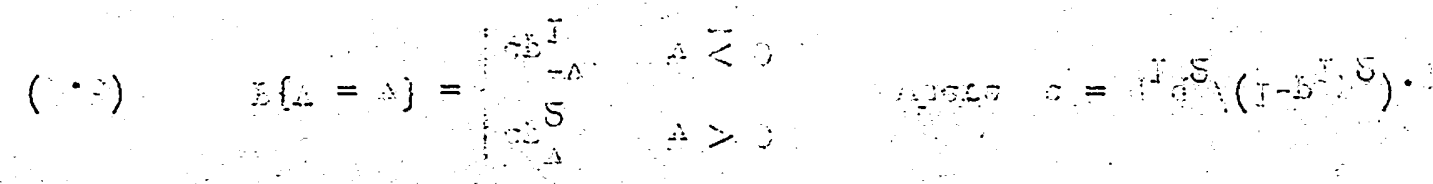


At this point we have two observations $U_{1}, U_{2}$, useful for estimating $\mathrm{P}_{1} \mathrm{P}_{2}$, plus independent "left over" information $\mathrm{V}_{2}$ which, like $\mathrm{V}_{1}$, can be combined with a $\operatorname{Geom}\left(\mathrm{p}_{1}\right)$ observation if $\mathrm{v}_{2}>0$ or with a $\operatorname{Geom}\left(\mathrm{p}_{2}\right)$ observation if $V_{2} \leq 0$. The procedure continues until either the $X^{\prime} s$ or the $Y^{\prime}$ 's are exhausted. As in the Lieberman-Ross method there will then be residual information about one parameter, but not about both. Suppose the procedure yields $r$ values $U_{j}$. These are combined without loss of information about $\mathrm{p}_{1} \mathrm{p}_{2}$ by forming the sum (5.6) $s_{r}=\sum_{i=1}^{r} U_{i}$.

Well known distribution theory gives $s_{r} \sim N B\left(r, p_{1} p_{2}\right)$, so that it becomes a straightforward problem to find confidence limits for $\mathrm{p}_{1} \mathrm{p}_{2}$ using the negative binomial distribution, as we show in Section 5.4 .

\subsection{A symmetric approximation.}

Some simplification is possible, and the results are more closely analogous to those of Lieberman and Ross, if we define the "symmetric approximation" to be that obtained by deleting the "-1" in the definitions (5.4) and (5.5) of $\mathrm{U}_{2}$ and $\mathrm{v}_{2}$. When $\mathrm{q}_{1}$ and $\mathrm{q}_{2}$ are sma11, then $\mathrm{V}=0$ has sma11 probability, and the "symmetric approximation" is presumably appropriate. Let us define the following partial sums:

$$
\begin{array}{rlr}
\mathrm{T}_{1 j} & =\sum_{i=1}^{j} x_{i} & \mathrm{j}=1, \ldots, \mathrm{m} \\
(5.7) & \mathrm{T}_{2 j}=\sum_{i=1}^{j} \mathrm{Y}_{i} & \mathrm{j}=1, \ldots, \mathrm{n} \\
\mathrm{s}_{j} & =\sum_{i=1}^{j} \mathrm{U}_{i} & \mathrm{j}=1, \ldots, \mathrm{r}^{\prime}
\end{array}
$$




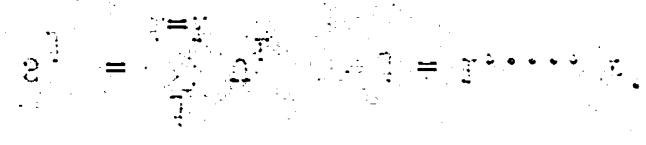

$(\because) \quad \because^{5}=\frac{1}{3} \quad=\cdots \cdots \cdots$

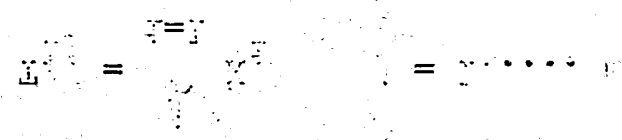

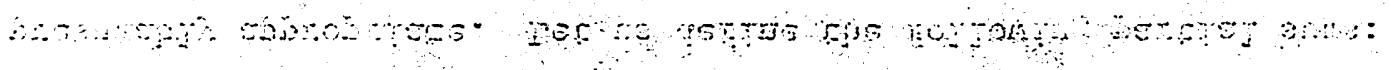

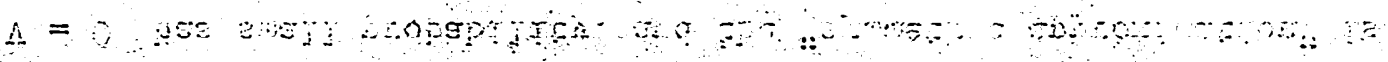

$\left.(\because),()^{\circ}\right)$ o

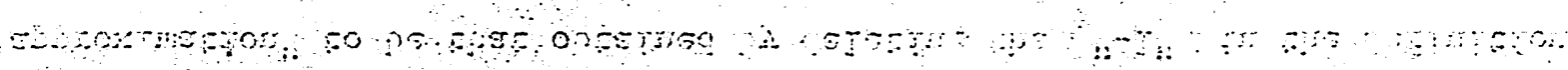

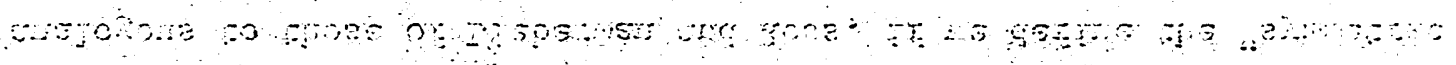

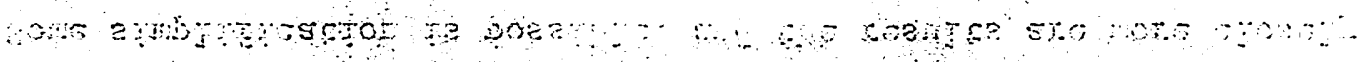

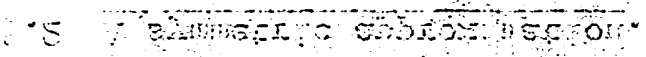

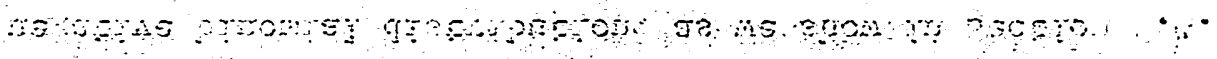

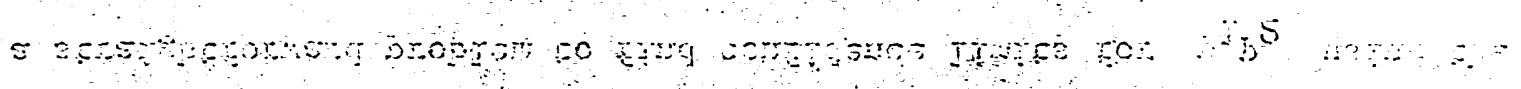

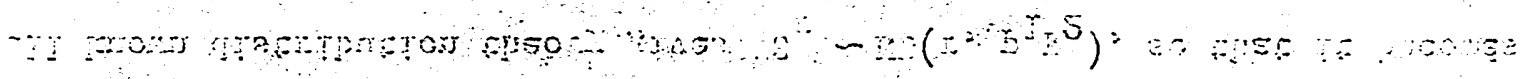

$$
(\cdots) \quad a^{-}=a^{r=z}
$$

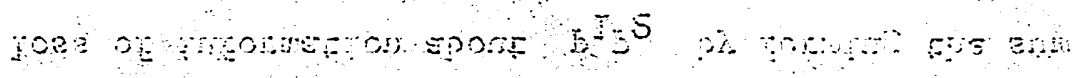

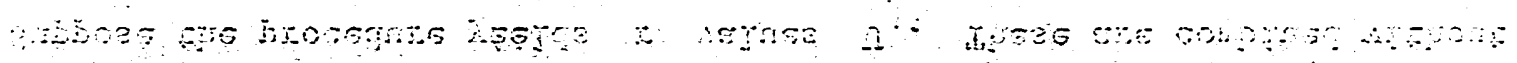

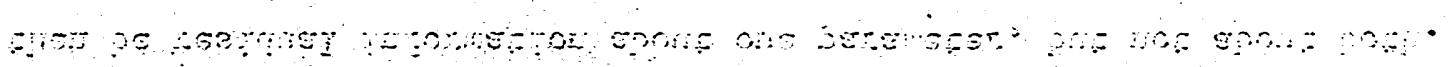

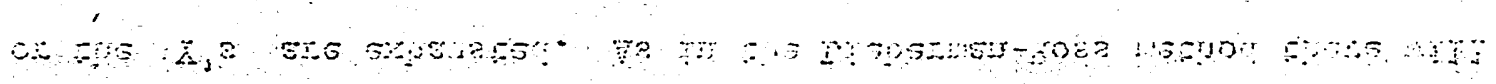

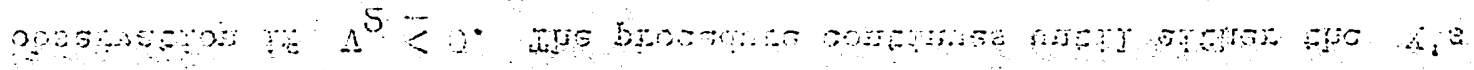

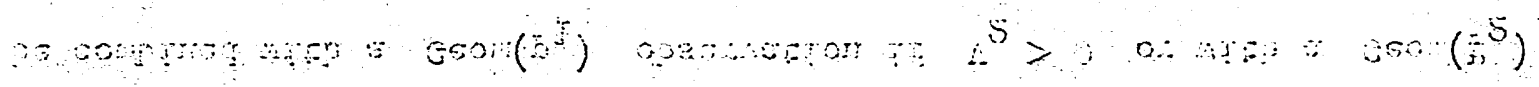

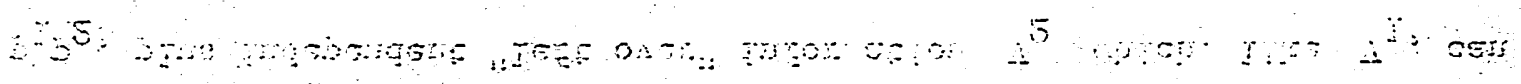

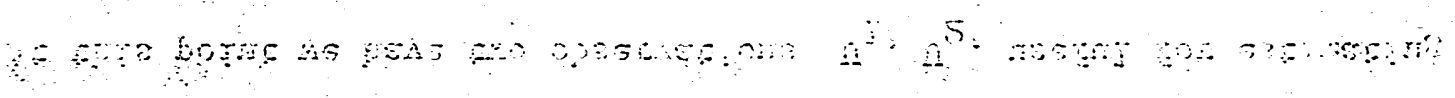


where $r^{\prime}$ is the number of $U$-values provided by the symmetric approximation. As in the Lieberman-Ross procedure, it can be shown that the construction is such that the sequence of $S_{j}$ 's agrees exactly with the first $r$ terms obtained by putting the $\mathrm{T}_{1 j}$ 's and $\mathrm{T}_{2 j}$ 's into a single ordered sequence (an example is given in section 5.3). Therefore $W_{r^{\prime}}$ can be calculated simply by

(5.8) $\quad \mathrm{S}_{\mathrm{r}^{\prime}}=\min \left(\mathrm{T}_{1 \mathrm{~m}}, \mathrm{~T}_{2 \mathrm{n}}\right)$

and $r^{\prime}$ is the number of $T_{1 j}$ 's and $T_{2 j}$ 's less than or equal to $S_{r^{\prime}}$. This approximation shows that when $q_{1}$ and $q_{2}$ are small there will be a minimum of "left over" information, so that the procedure has a high efficiency, when we have approximately the same number of Bernoulli observations from the two populations. Of course, it is also possible to deliberately terminate sampling in such a way as to minimize "left over" information.

Because of the simplifications arising in the symmetric approximation, it is tempting to think that it may actually be exact. A simple check shows that unfortunately it is not. Let $A=\left\{X_{1}=Y_{1}=0\right\}, B=\left\{X_{1}=X_{2}=0\right\}$, $\mathrm{C}=\left\{\mathrm{Y}_{1}=\mathrm{Y}_{2}=0\right\}$. Then with the modified definition of $\mathrm{U}_{2}$, $\left\{\mathrm{U}_{1}=\mathrm{U}_{2}=0\right\}=\mathrm{A} \cup \mathrm{B} \cup \mathrm{C}$. Since $\mathrm{BC}=\mathrm{ABC}, \mathrm{P}\{\mathrm{A} \cup \mathrm{B} \cup \mathrm{C}\}=\mathrm{P}(\mathrm{A})+\mathrm{P}(\mathrm{B})+\mathrm{P}(\mathrm{C})$ $-P(A B)-P(A C)=q_{1} q_{2}+q_{1}^{2}+q_{2}^{2}-q_{1}^{2} q_{2}-q_{1} q_{2}^{2}$. When $q_{1}=q_{2}=q=1-p$, this gives $P\left\{U_{1}=U_{2}=0\right\}=q^{2}(1+2 p)$. But if $U_{1}$ and $U_{2}$ are independent Geom $\left(p^{2}\right)$ variates, $P\left\{U_{1}=U_{2}=0\right\}=\left(1-p^{2}\right)^{2}=q^{2}\left(1+2 p+p^{2}\right)$, so that the symmetric approximation is not exact.

\subsection{Examples.}

To illustrate a case where $p_{1}$ and $p_{2}$ are close to unity, let $\left(x_{1}, \ldots, x_{3}\right)=(24,35,39),\left(Y_{1}, \ldots, Y_{6}\right)=(16,12,27,12,43,19)$. 


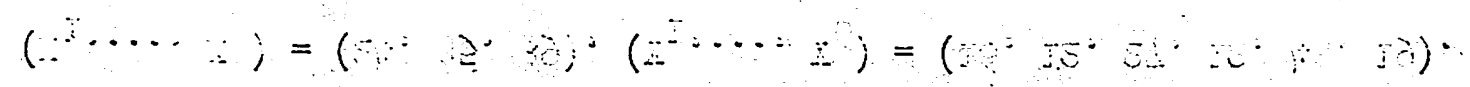

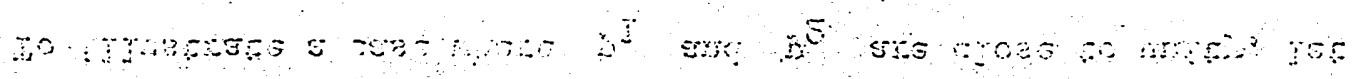

$\cdots$

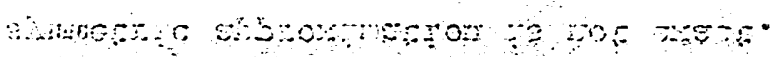

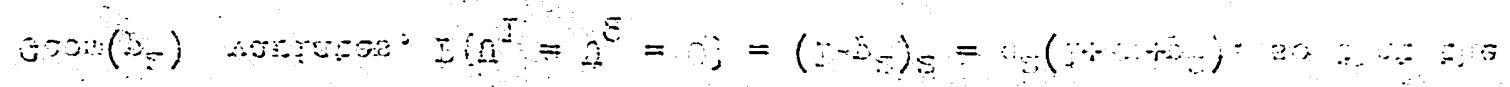

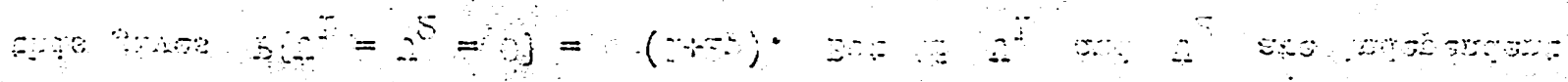

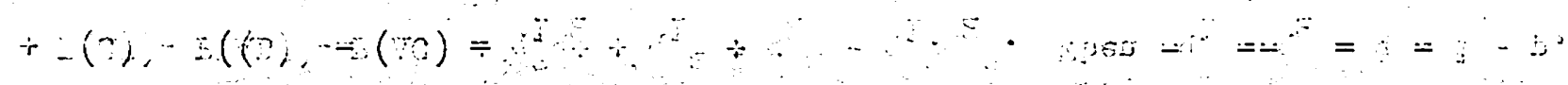

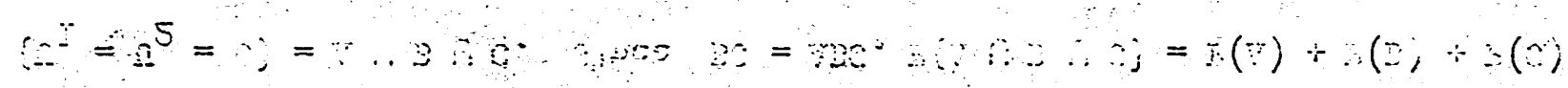

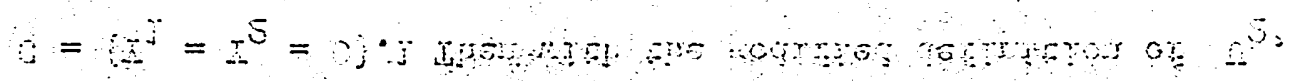

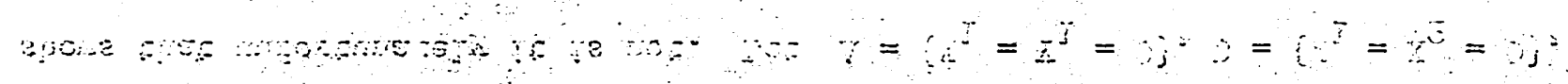

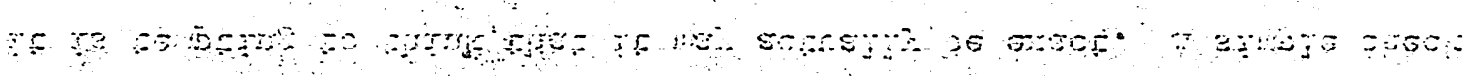

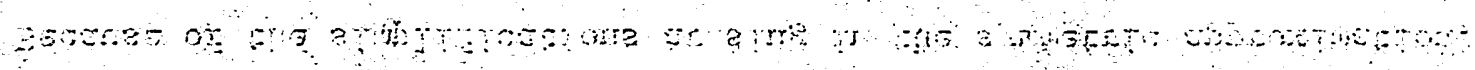

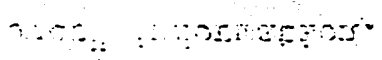

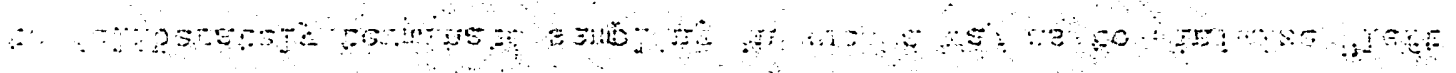

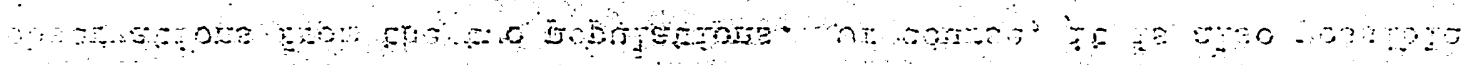

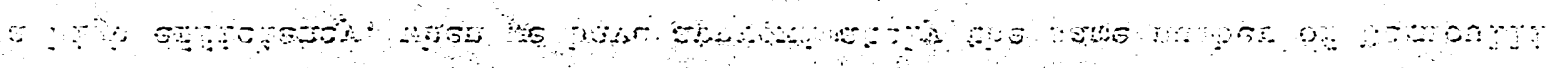

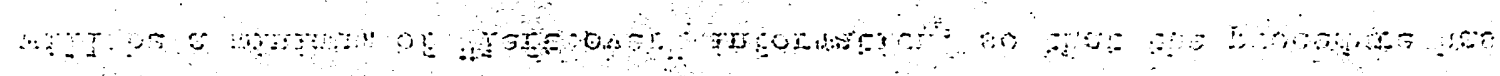

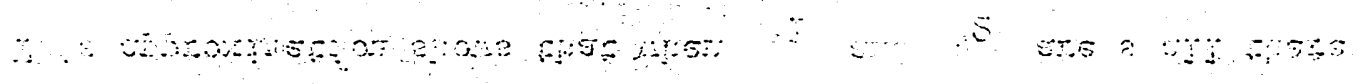

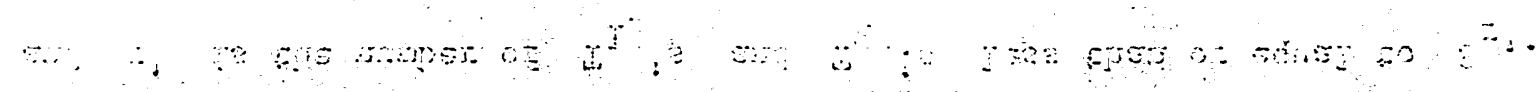

$(\because) \quad \therefore=\left(n^{2}\right)$

अ......

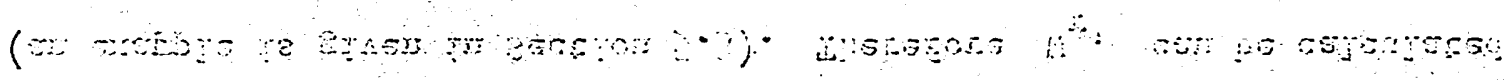

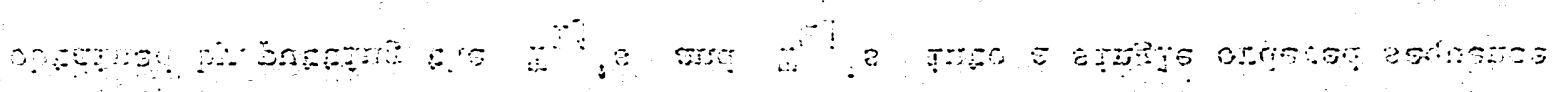

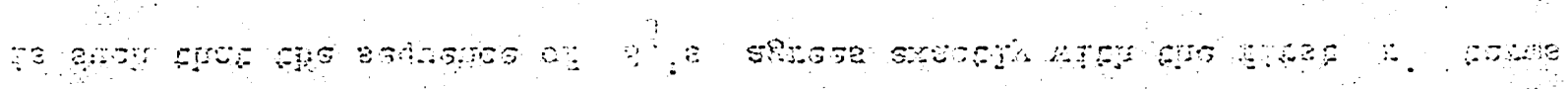

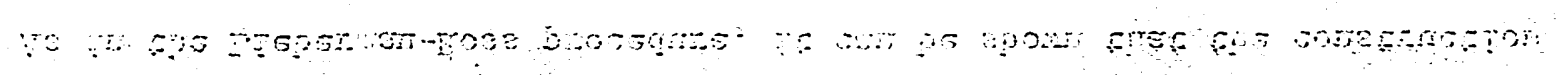

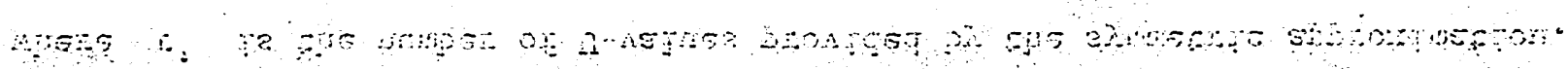


$\mathrm{U}_{1}=\min (24,16)=16, \mathrm{v}_{1}=16-24=-8$, and we take +8 to be a $\operatorname{Geom}\left(\mathrm{p}_{1}\right)$ observation. $\mathrm{U}_{2}=\min (8,12)=8, \mathrm{v}_{2}=12-8=4$, and we take $4-1=3$ to be a Geom $\left(p_{2}\right)$ observation. Similarly we find: $\left(v_{1}, \ldots, v_{7}\right)=(-8,4,-32,-5,7,-33,10),\left(U_{1}, \ldots, U_{7}\right)=(16,8,3$, $27,5,6,33),\left(s_{1}, \ldots, s_{7}\right)=(16,24,27,54,59,65,98)$. The "left over" information is $\mathrm{V}_{7}=10$ and $\mathrm{Y}_{6}=19$. The symmetric approximation would give $\left(s_{1}, \ldots, s_{7}\right)=(16,24,28,55,59,67,98)$, where $s_{1}=Y_{1}$, $S_{2}=X_{1}, S_{3}=Y_{1}+Y_{2}, S_{4}=Y_{1}+Y_{2}+Y_{3}$, etc. We know $s_{7} \sim N B\left(7, P_{1} p_{2}\right)$, and the value $\mathrm{s}_{7}=98$ summarizes the information about $\mathrm{p}_{1} \mathrm{p}_{2}$. In effect, we have terminated with the seventh failure on the 105-th Bernoulli trial. The maximum likelihood estimator of $p_{1} p_{2}$ based on $\mathrm{S}_{7}$ is $98 / 105$, and (as we show in section 5.4) a 90 percent confidence interval for $\mathrm{p}_{1} \mathrm{p}_{2}$ is $(0.890,0.968)$.

With $P_{1}$ and $P_{2}$ smal1, we might get observations like $\left(x_{1}, \ldots, x_{7}\right)=(0,1,1,0,0,2,1)$ and $\left(Y_{1}, \ldots, Y_{12}\right)=(0,0,1,0,1$, $1,0,2,0,0,1,2)$. Our asymmetrical solution gives $U_{6}=U_{10}=U_{17}=1$ and all other $U$-values equal to zero. Left over information is $x_{7}=1$ and $\nabla_{17}=-1$. The maximum likelihood estimator of $p_{1} p_{2}$ based on $\mathrm{S}_{17}=3$ is $3 / 20=0.15$. The symmetric approximation gives a substantially different sequence of $U$ values, and in fact direct comparison of the methods is difficult because the asymmetric solution tends to use a greater proportion of $\mathrm{Y}$ values, so that at termination the two methods have used different sets of data. We believe the symmetric approximation will tend to overestimate $\mathrm{p}_{1} \mathrm{p}_{2}$ when $\mathrm{p}_{1}$ and $\mathrm{p}_{2}$ are sma11. In the above sequence $\left(x_{1}, \ldots, x_{6}\right)$ we have 4 successes and 6 failures, giving $\hat{p}_{1}=0.4$. In $\left(Y_{1}, \ldots, Y_{12}\right)$ we have 8 successes and 


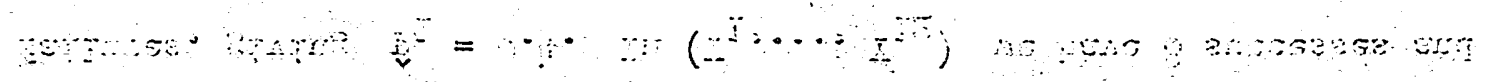

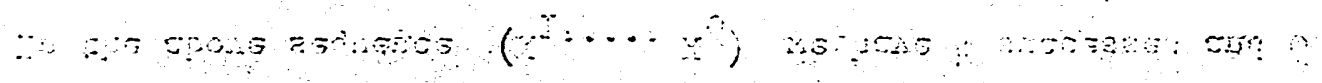

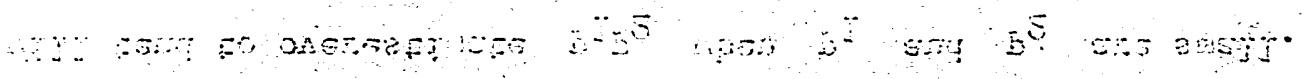

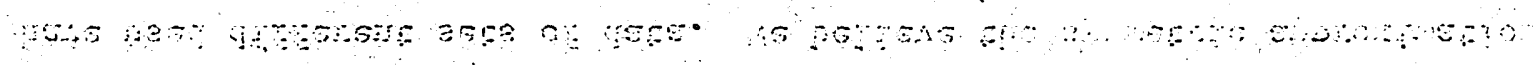

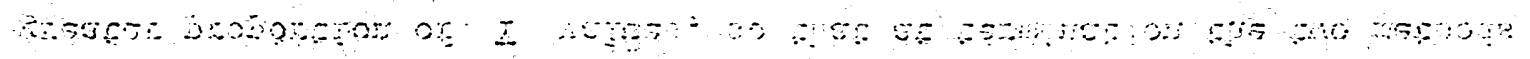
and a a 6.

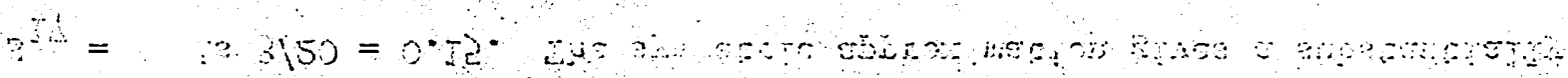

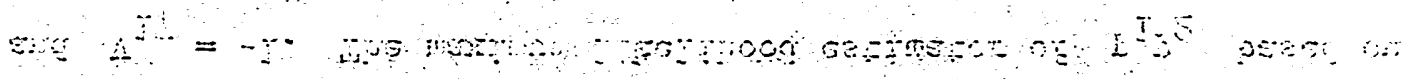

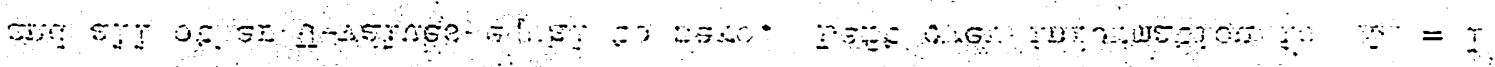

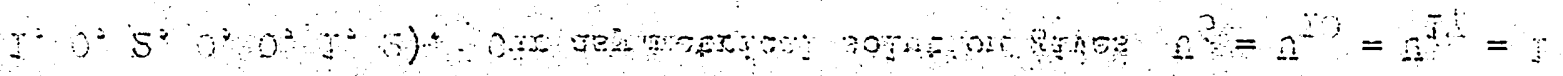

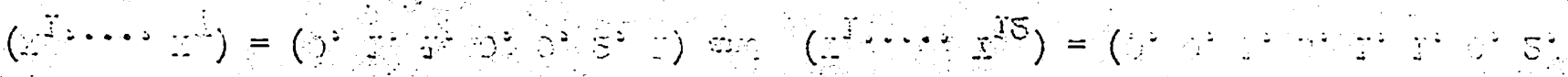

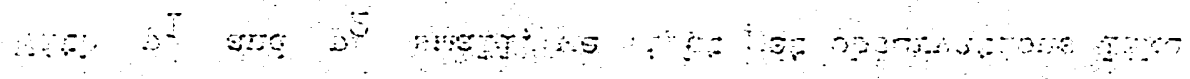

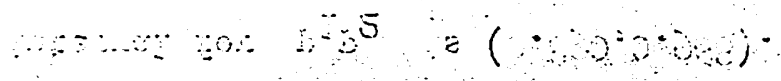
अ a

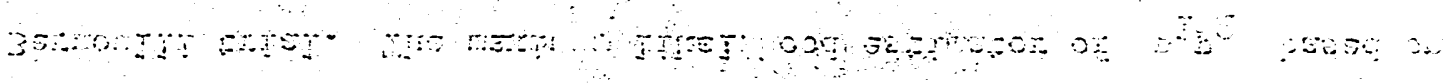

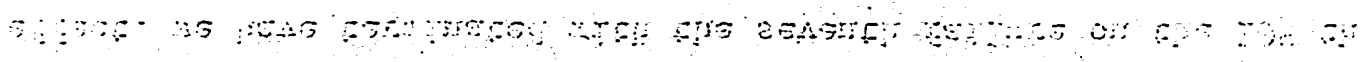

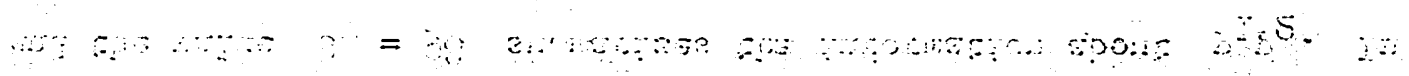

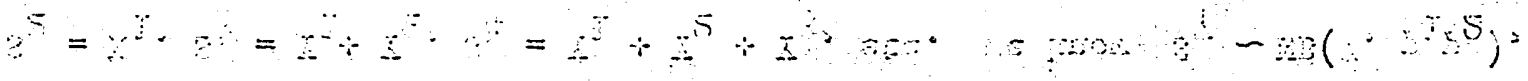

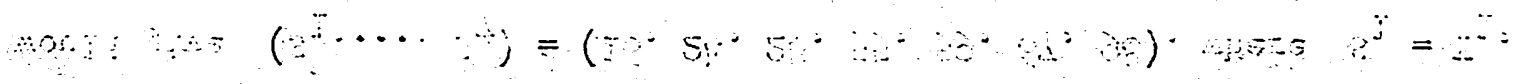

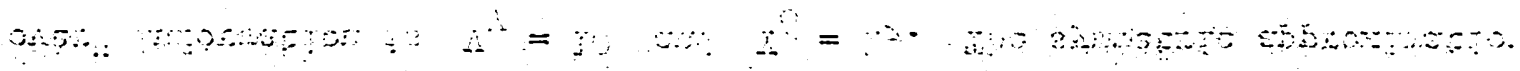

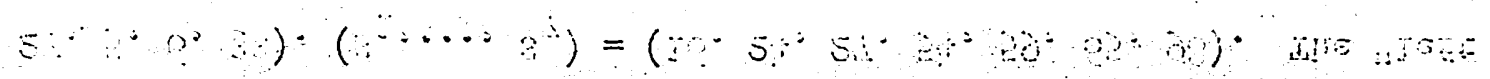

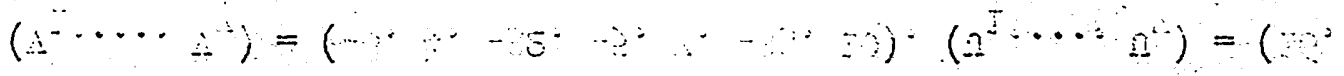

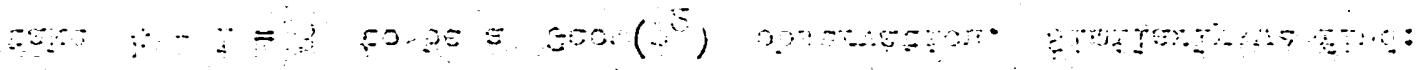

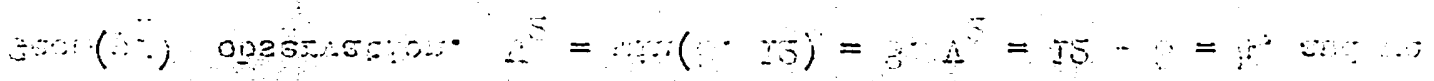

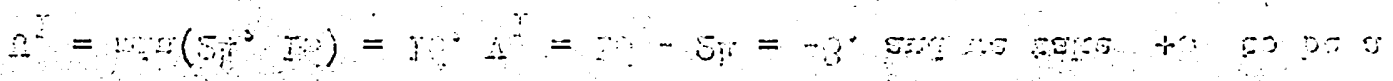


12 failures, giving $\hat{\mathrm{p}}_{2}=0.4$. The value $\hat{\mathrm{p}}_{1} \hat{\mathrm{p}}_{2}=0.16$ agrees well with the value 0.15 derived above. We give this comparison simply as a partial check, and not to claim any superior method of point estimation. The point of the procedure is rather the possibility of finding "exact" confidence limits for $\mathrm{P}_{1} \mathrm{P}_{2}$. In the next section we find a 90 percent confidence interval $(0.044,0.344)$.

5.4 Calculation of negative binomial confidence limits.

Let us denote by $\mathrm{F}_{\mathrm{X}}(\mathrm{x} ; \mathrm{r}, \mathrm{p})$ the cumulative distribution function of $\mathrm{X} \sim \mathrm{NB}(\mathrm{r}, \mathrm{p})$ defined in (2.1). Then using standard arguments

(5.8) $\quad \mathrm{P}\{\mathrm{p} \leq \overline{\mathrm{p}}(\mathrm{x})\} \geq \gamma$ and $\mathrm{P}\{\underline{\mathrm{p}}(\mathrm{x}) \leq \mathrm{p}\} \geq \gamma$

when $\bar{p}(x)$ and $\underline{p}(x)$ are solutions of

(5.9) $\quad \mathrm{F}_{X}(\mathrm{x} ; \mathrm{r}, \overline{\mathrm{p}})=1-\gamma$ and $\mathrm{F}_{X}(\mathrm{x}-1 ; r, \underline{\mathrm{p}})=\gamma$.

It is known [7], [15] that the negative binomial is related to the incomplete beta function defined in (2.37) by

(5.10) $\quad \mathrm{F}_{\mathrm{X}}(\mathrm{x} ; \mathrm{r}, \mathrm{p})=\mathrm{I}_{1-\mathrm{p}}(\mathrm{r}, \mathrm{x}+1)$.

Therefore we may also define $\bar{p}$ and $\underline{p}$ as solutions of

$$
I_{1-\underline{p}}(r, x+1)=1-\gamma \text { and } I_{1-\underline{p}}(r, x)=\gamma
$$

Thus the confidence limits could be found from tables of the incomplete beta function [17]. It is usually more convenient however to use the F distribution. The relationship here (see for example [16], p. 33) is (5.12) $\quad P\left\{F_{2 n}^{2 m} \geq F_{0}\right\}=I_{c}(n, m), \quad c=m /\left(m+n F_{0}\right)$, where of course $F_{2 \mathfrak{n}}^{2 m}$ is the Fisher-Snedecor $F$ variate with $2 m$ and 2n degrees of freedom. Thus we find 


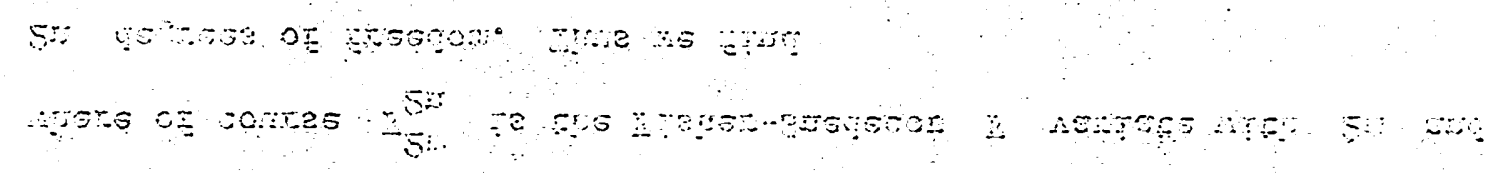

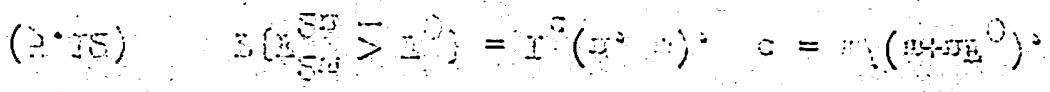

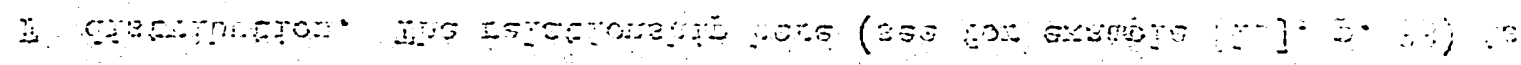

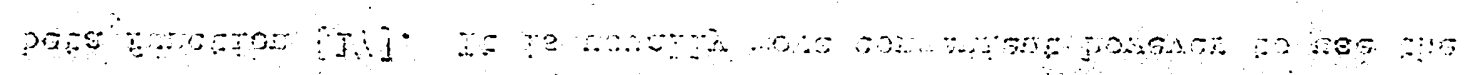

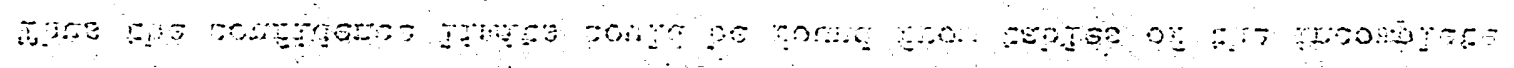

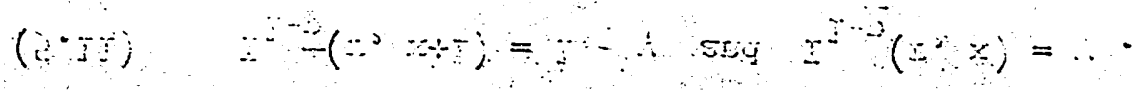

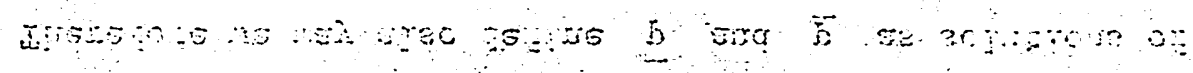

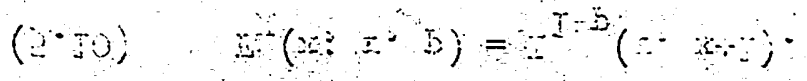

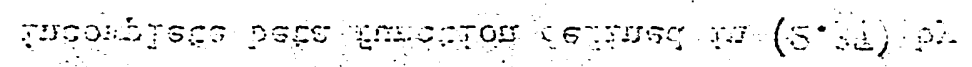

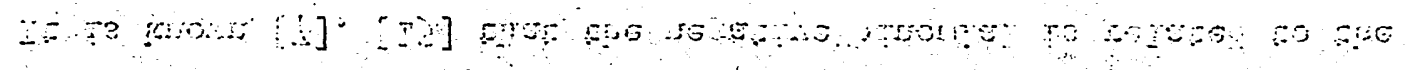

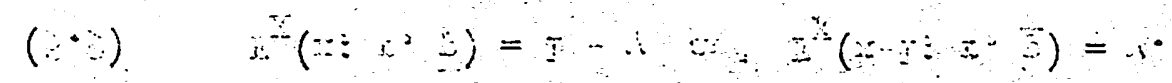

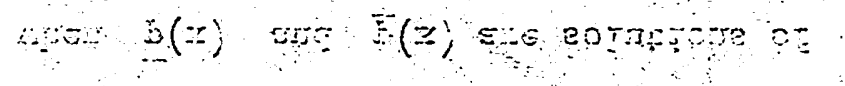

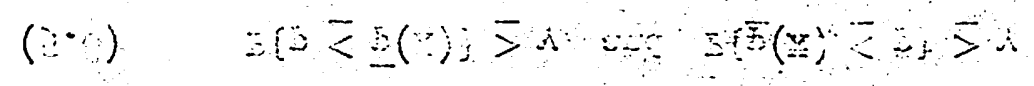

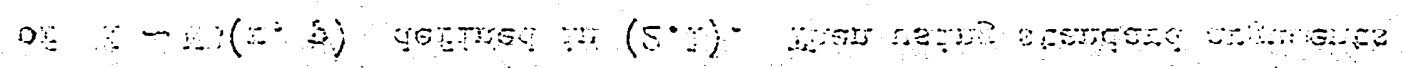

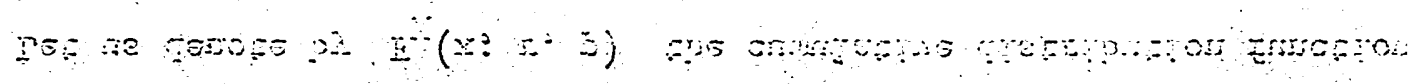

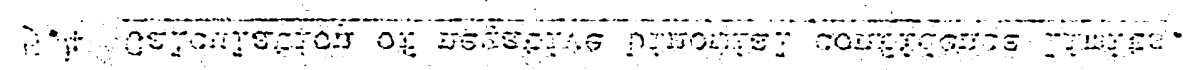

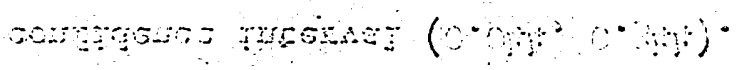

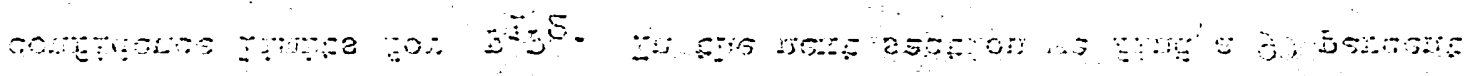

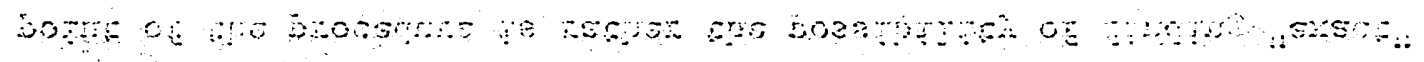

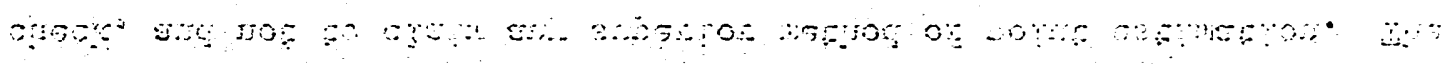
आ6

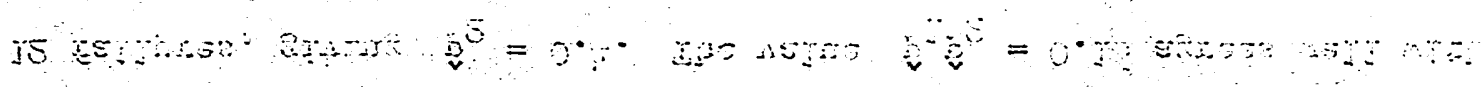


(5.13) $\quad \bar{p}=\frac{(x+1) F_{2 r}^{2 x+2}(\gamma)}{r+(x+1) F_{2 r}^{2 x+2}(\gamma)}, \quad \underline{p}=\frac{x F_{2 r}^{2 x}(1-\gamma)}{r+x F_{2 r}^{2 x}(1-\gamma)}$

where $F_{n}^{m}(\beta)$ is the $F$-percentile defined by

$$
P\left\{F_{n}^{m} \leq F_{n}^{m}(\beta)\right\}=\beta \text {. }
$$

Since $F$ tables usually give upper tails, we may use the standard trick of taking reciprocals to express $\underline{p}$ as

$$
\underline{p}=\frac{x}{x+r F_{2 x}^{2 r}(\gamma)} \text {. }
$$

If neither the incomplete beta nor the $F$ table is convenient, a number of approximations are available [1], [7].

For the first example of section 5.3, $r=7, x=98, F_{14}^{198}(0.95)=2.16$, $\mathrm{F}_{196}^{14}(0.95)=1.74$, and we find a 90 percent confidence interval $(\underline{p}, \bar{p})=(0.890,0.968)$. For the second example $r=17, x=3$, $\mathrm{F}_{34}^{8}(0.95)=2.23, \mathrm{~F}_{6}^{34}(0.95)=3.79$, and $(\underline{p}, \overline{\mathrm{p}})=(0.044,0.344)$ is

a 90 percent confidence interval.

\subsection{Estimation of $\underline{p}_{1} \underline{p}_{2} \ldots p_{k}$.}

There are many ways to extend the above procedures in order to estimate the product of $k$ parameters. If we consider the symmetric approximation and let $T_{i n_{i}}$ denote the cumulative sum of all $n_{i}$ geometric observations from population $i$, then $S_{r^{\prime}}=\min _{i}\left(T_{i n_{i}}\right)$ is approximately $N B\left(r^{\prime}, p_{1} p_{2} \cdots p_{k}\right)$ where $r^{\prime}$ is the number of cumulative sums $T_{i j}\left(j=1, \ldots, n_{i}\right)$ less than or equal to $S_{r}$. This analog of an exact result of Lieberman and Ross would appear to be a somewhat questionable approximation for large k. Perhaps the simplest exact procedure is a $(k-1)$-fold iteration of our two-population procedure. Let $\left(\mathrm{x}_{i 1}, \ldots, \mathrm{x}_{\mathrm{in}_{i}}\right)$ denote $\operatorname{Geom}\left(\mathrm{p}_{i}\right)$ 


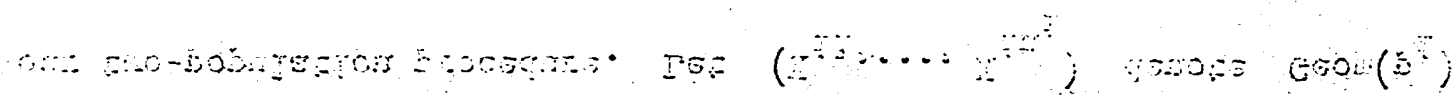

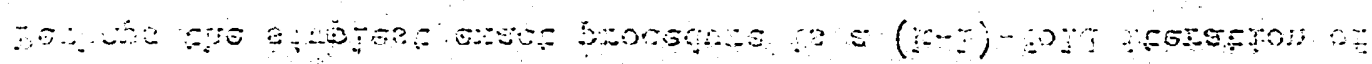

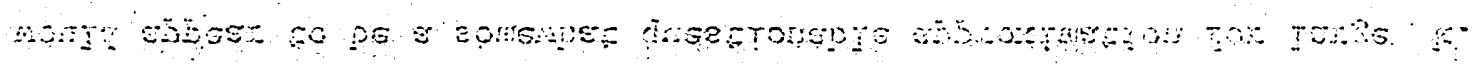

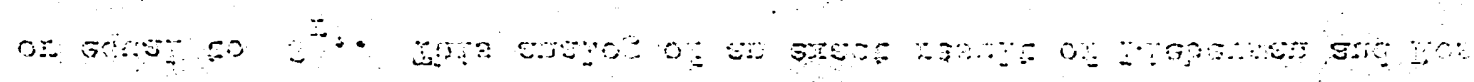

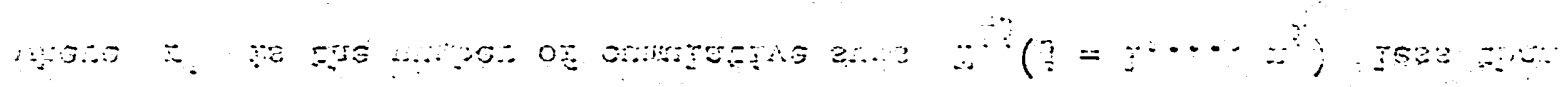

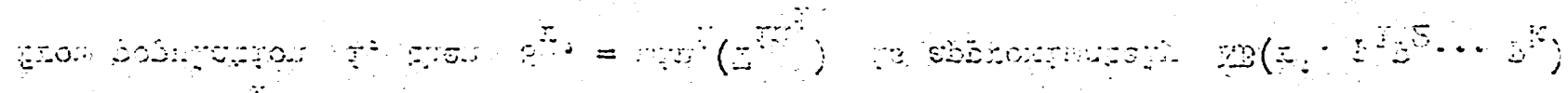
re

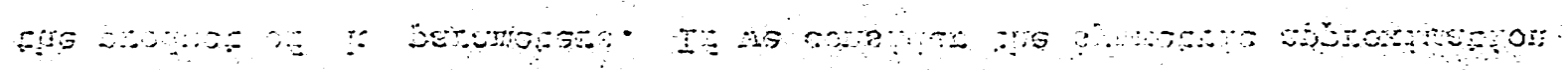

4. is

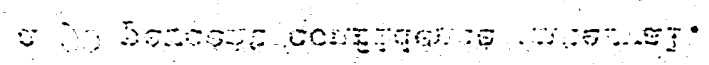

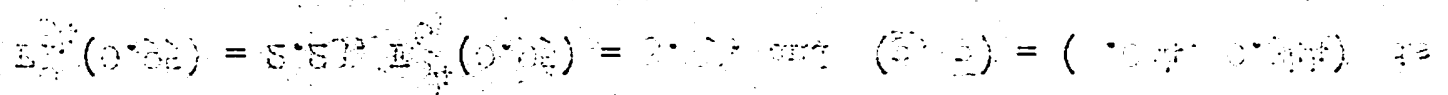

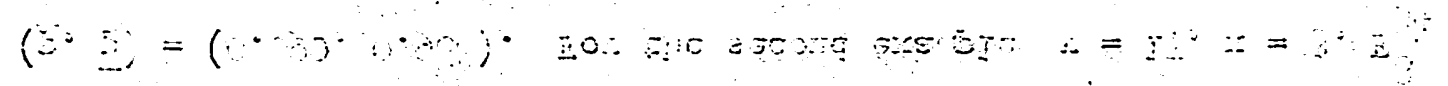

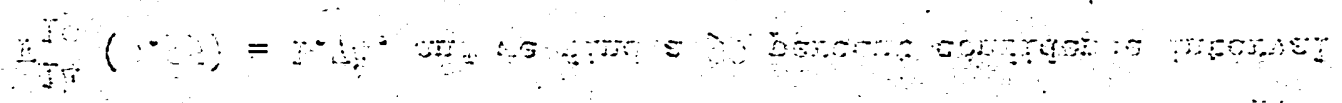

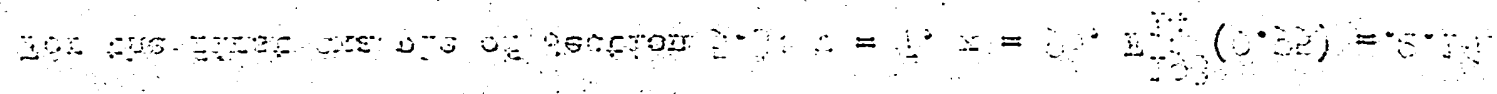

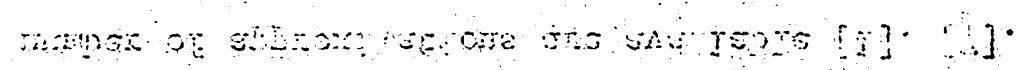

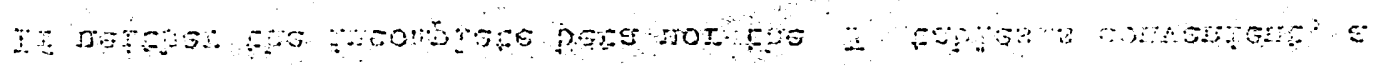

$(a) \quad y=\frac{x+x^{5}(x)}{x}$

Dy :

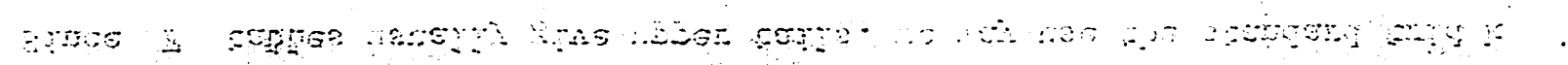

$$
\because\left[\begin{array}{c}
2 \\
3
\end{array}(y)=\right.
$$

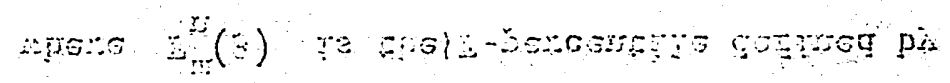

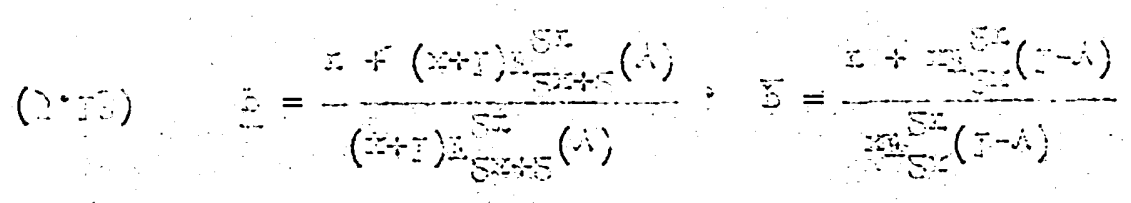


observations. Utilizing observations with $i=1$, 2 , we may construct by the method of Section 5.1 , values $U_{1}, \ldots, U_{r}$ which are independent Geom $\left(\mathrm{p}_{1} \mathrm{p}_{2}\right)$ variates. Next we combine $\mathrm{U}_{1}, \ldots, \mathrm{U}_{\mathrm{r}}$ with $\mathrm{x}_{31}, \ldots, \mathrm{x}_{3 \mathrm{n}_{3}}$ to obtain $\operatorname{Geom}\left(\mathrm{p}_{1} \mathrm{p}_{2} \mathrm{p}_{3}\right)$ variates, and so on.

Other procedures can be described which involve choosing the minimum of more than two geometric variates. It does not seem worthwhile to spell out details here, but we will state without proof three lemmas giving distribution theory which could be used to this end. These lemmas may be of some interest in themselves, and could possibly be shown to yield procedures in some way superior to the method of iteration.

Let $x_{1}, \ldots, x_{k}$ be independent, $x_{i} \sim \operatorname{Geom}\left(p_{i}\right)$, and define (5.15) $\quad \mathrm{w}_{0}=\min \left(\mathrm{x}_{1}, \ldots, \mathrm{x}_{k}\right), \quad \mathrm{w}_{i}=\mathrm{x}_{i+1}-\mathrm{x}_{i}, \quad i=1, \ldots, k-1$. Lemma 5.4.

$\mathrm{w}_{0} \sim \operatorname{Geom}\left(\mathrm{p}_{1} \ldots \mathrm{p}_{\mathrm{k}}\right)$ and $\mathrm{w}_{0}$ is independent of $\left(\mathrm{w}_{1}, \ldots, \mathrm{w}_{\mathrm{k}-1}\right)$. Lemma 5.5 .

$\left\{\mathrm{w}_{0}=\mathrm{w}_{0}\right\}$ is independent of $\left\{\mathrm{x}_{1} \leq \mathrm{x}_{2} \leq \cdots \leq \mathrm{x}_{\mathrm{k}}\right\}$

Lemma 5.6.

Given that $x_{1} \leq x_{2} \leq \ldots \leq x_{k}, w_{0}, w_{1}, \ldots, w_{k-1}$ are mutually

independent and $w_{i} \sim \operatorname{Geom}\left(p_{i+1} \cdots p_{k}\right)$ for $i=0,1, \ldots, k-1$. 


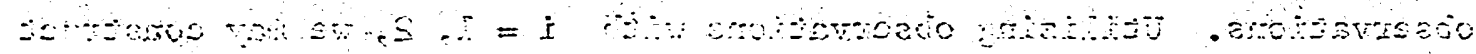

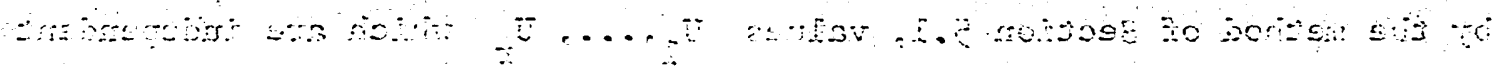

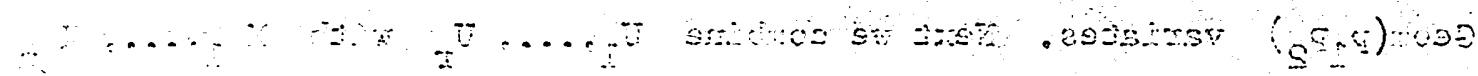

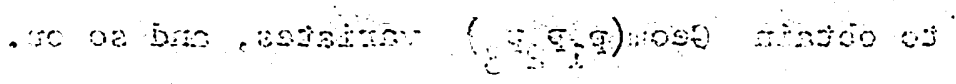

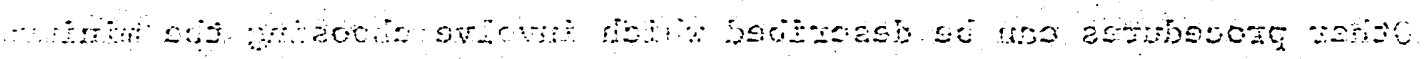

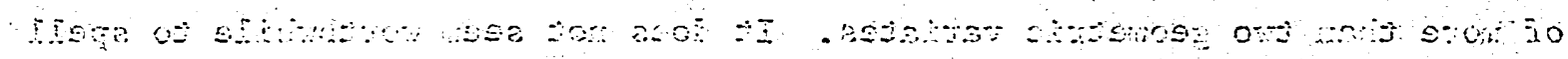

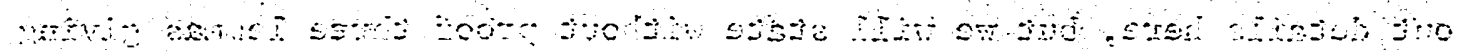

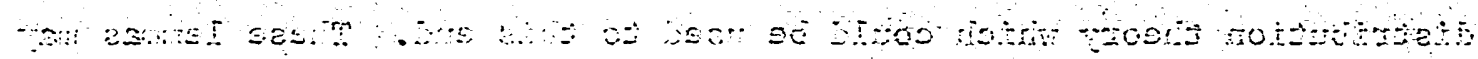

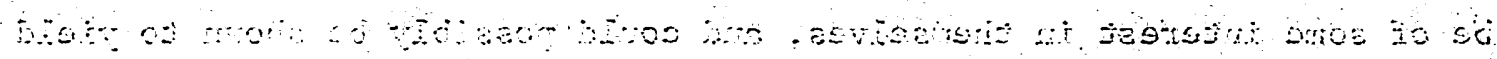

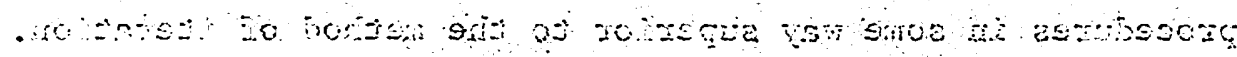

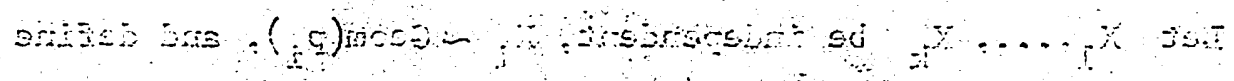

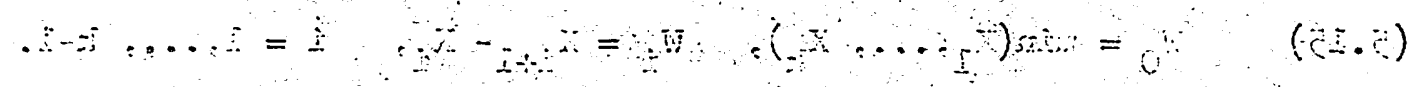

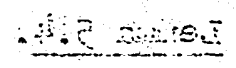

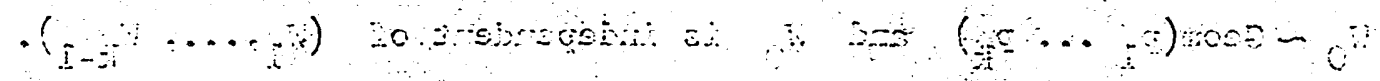

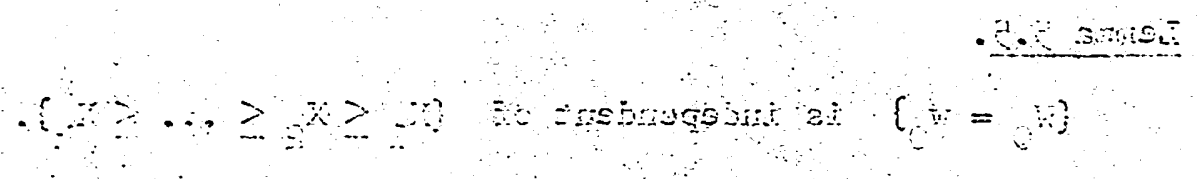

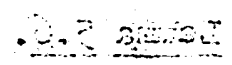

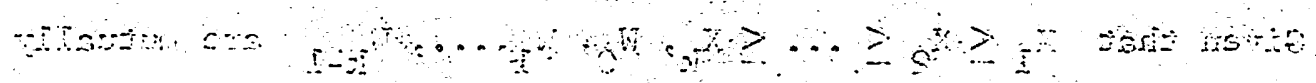

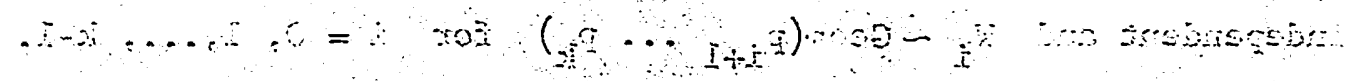




\section{REFERENCES}

[1] Bartko, John, J., "Approximating the Negative Binomia1," Technometrics, $8(1966), 345-50$.

[2] Buehler, Robert J., "Confidence Limits for the Product of Two Binomial Parameters," Journal of the American Statistical Association, 52 (1957), 482-93.

[3] Feller, William, An Introduction to Probability Theory and Its Applications, Vo1. 1, 3rd ed., New York: John Wiley and Sons, Inc., 1968.

[4] Ferguson, T. S., "A Characterization of the Geometric Distribution," American Mathematical Monthly, 72 (1965), 256-60.

[5] Harris, Bernard, "Hypothesis Testing and Confidence Intervals for Products and Quotients of Poisson Parameters with Applications to Reliability," Journal of the American Statistical Association, 66 (1971), 609-13.

[6] Hwang, Dar-Shong, "Interval Estimation of Functions of Bernoul1i Parameters with Reliability and Biomedical Applications," University of Minnesota $\mathrm{PhD}$ Thes is and School of Statistics Technical Report No. 152, Minneapolis, 1971.

[7] Johnson, Norman L. and Kotz, Samuel, Discrete Distributions, Boston: Houghton Mifflin Co., 1969.

[8] Lehmann, E. L., Testing Statistica1 Hypotheses, New York: John Wiley and Sons, Inc., 1959.

[9] Lehmann, E. L. and Scheffé, Henry, "Completeness, Similar Regions, and Unbiased Estimation, Part II," Sankhyā, 15 (1955), 219-36. 


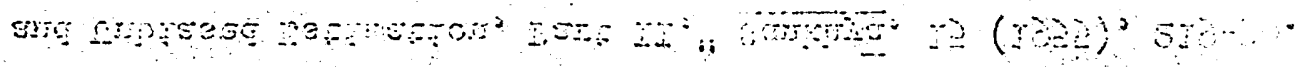

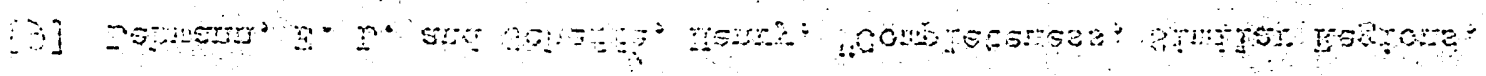

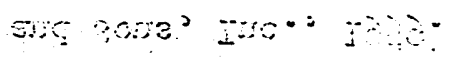

(1)

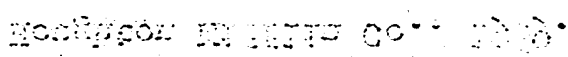

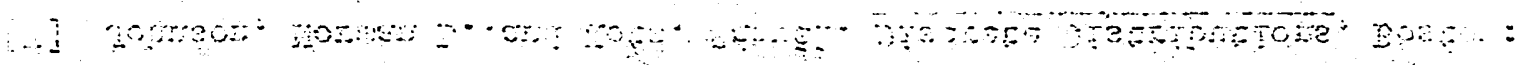

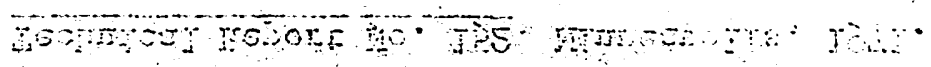

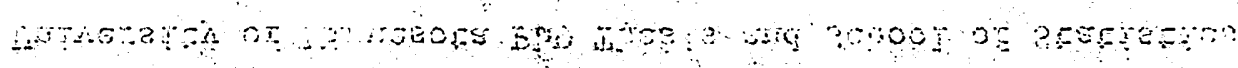

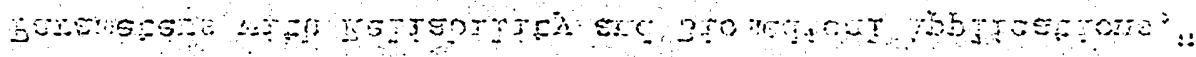

¿ Q co

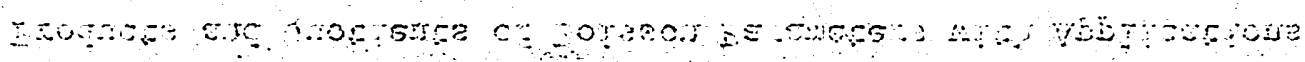

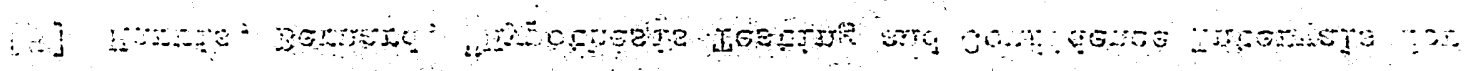

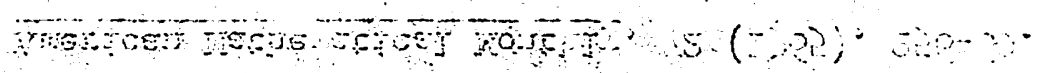

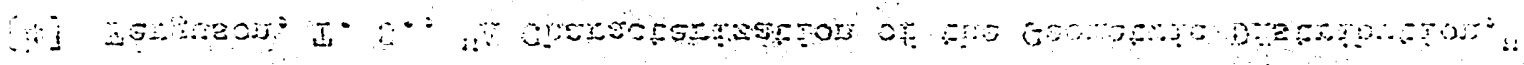
5.

poserposa

[1] [$(20) \cdot 95 \cdot 9$

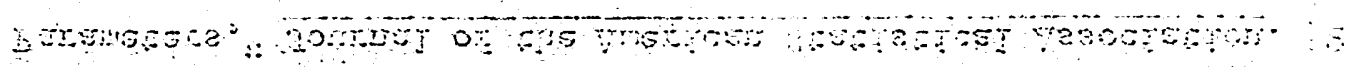

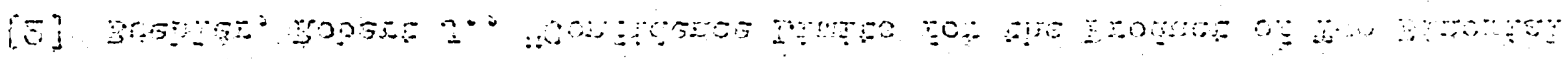
$8(n+\infty)^{2}, 9-n$

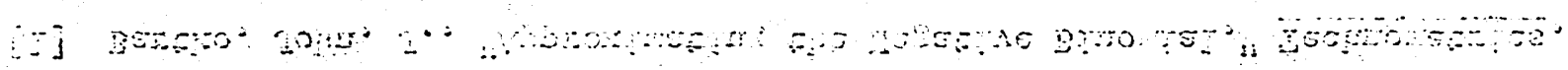
and 
[10] Lentner, M. M. and Buehler, R. J., "Some Inferences about Gamma Parameters with an Application to a Reliability Problem," Journal of the American Statistical Association, 58 (1963), 670-7.

[11] Lieberman, Gerald J. and Ross, Sheldon M., "Confidence Intervals for Independent Exponential Series Systems," Technical Report No. 130, Department of Operations Research and Department of Statistics, Stanford University, Stanford, California, 1970. (To appear in Journal of the American Statistical Association.)

[12] Madansky, Albert, "Approximate Confidence Limits for the Reliability of Series and Parallel Systems," Technometrics, 7 (1965), 495-503.

[13] Mann, Nancy R., "Computer-Aided Selection of Prior Distributions for Generating Monte Carlo Confidence Bounds on System Reliability," Nava1 Research Logistics Quarterly, 17 (1970), 41-54.

[14] Myhre, Janet and Saunders, Sam C., "On Confidence Limits for the Reliability of Systems," The Annals of Mathematical Statistics, 39 (1968), 1463-72.

[15] Patil, G. P., "On the Evaluation of the Negative Binomial Distribution with Examples," Technometrics, 2 (1960), 501-5.

[16] Pearson, E. S. and Hartley, H. 0., Biometrika Tables for Statisticians, Volume I, Cambridge University Press, 1956.

[17] Pearson, Kar1, Tables of the Incomplete Beta-Function, Cambridge University Press, 1934.

[18] Rosenblatt, Joan Raup, "Confidence Limits for the Reliability of Complex Systems," Statistical Theory of Reliability, 115-37, Marvin Zelen, Ed., University of Wisconsin Press, Madison, 1963. 


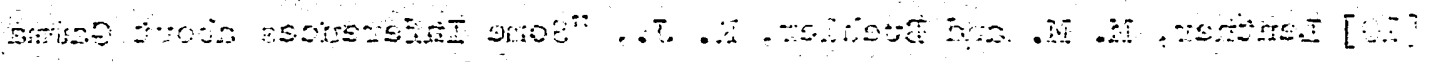

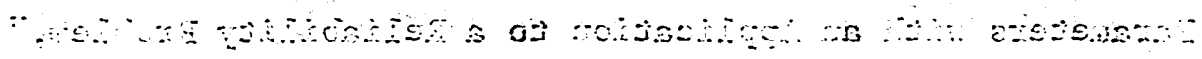

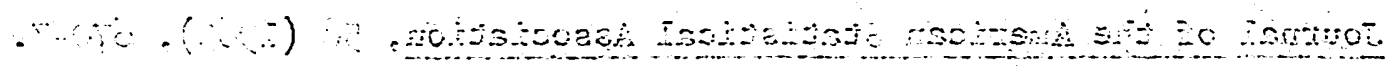

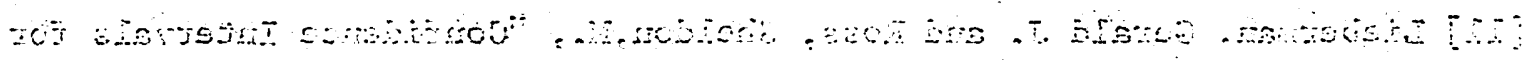

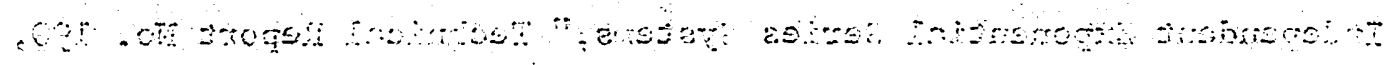

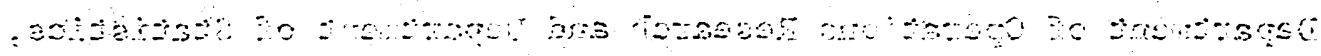

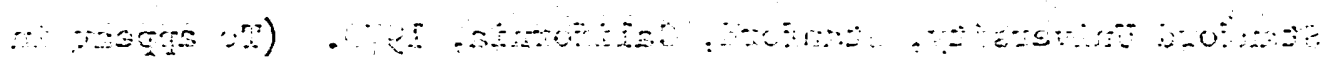

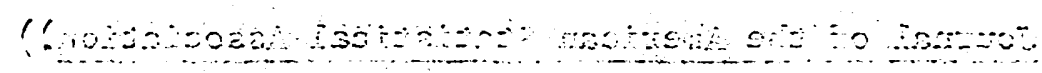

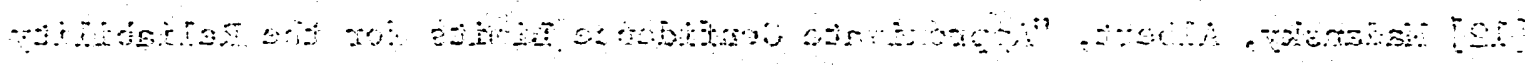

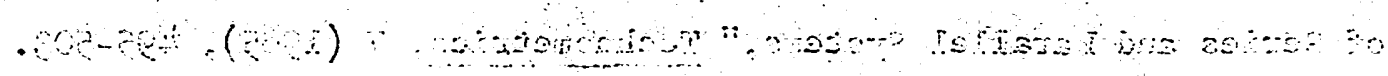

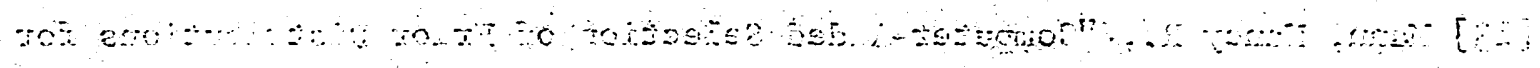

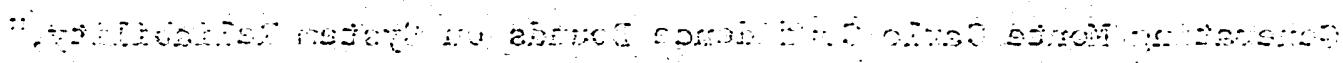

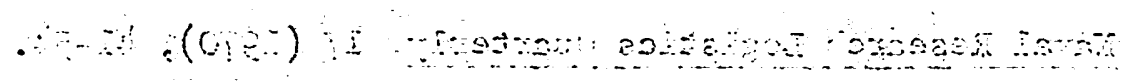

1 द 20 a do

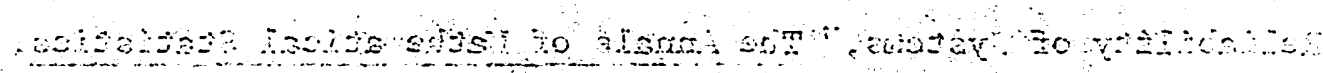

$$
-8 \mathrm{~T}-(\sec )
$$

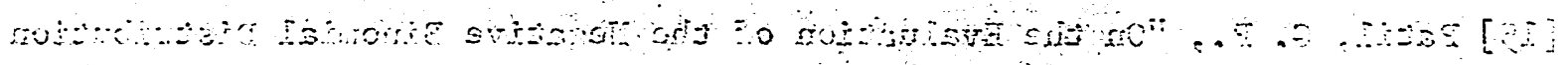

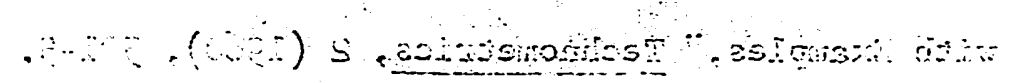

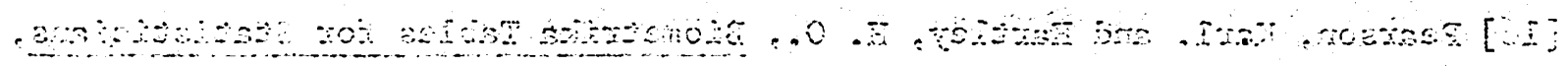

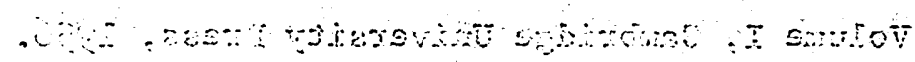

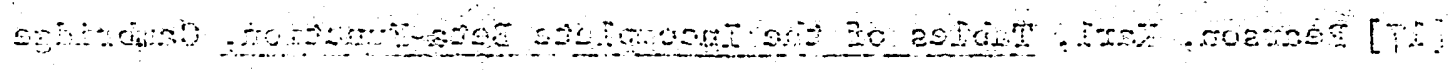

$\therefore \because$ a

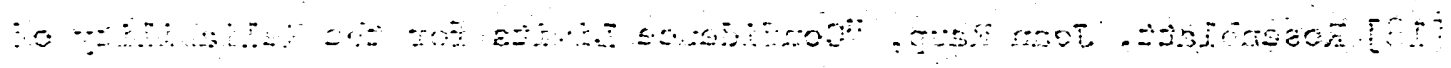

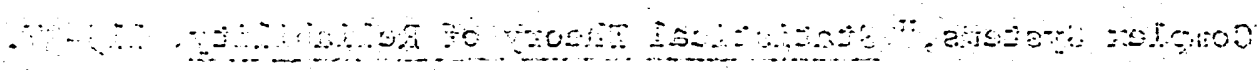

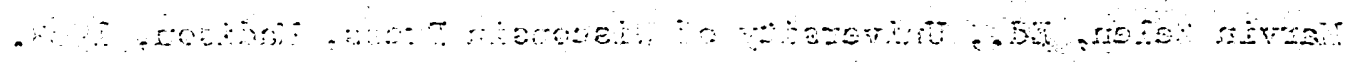


[19] Stevens, W. I., "Fiducial Limits of the Parameter of a Discontinuous Distribution," Biometrika, 37 (1950), 117-29.

[20] Whittaker, E. T. and Watson, G. N., A Course of Modern Analysis, 2nd ed. Cambridge, England: Cambridge University Press, 1915. 